Florida International University FIU Digital Commons

\title{
Structural Flexibility and Oxygen Diffusion Pathways in Monomeric Fluorescent Proteins
}

Chola K. Regmi

FLORIDA INTERNATIONAL UNIVERSITY, cholaregmi@gmail.com

DOI: $10.25148 /$ etd.FI14040821

Follow this and additional works at: https://digitalcommons.fiu.edu/etd

Part of the Biological and Chemical Physics Commons

\section{Recommended Citation}

Regmi, Chola K., "Structural Flexibility and Oxygen Diffusion Pathways in Monomeric Fluorescent Proteins" (2014). FIU Electronic Theses and Dissertations. 1122.

https://digitalcommons.fiu.edu/etd/1122

This work is brought to you for free and open access by the University Graduate School at FIU Digital Commons. It has been accepted for inclusion in FIU Electronic Theses and Dissertations by an authorized administrator of FIU Digital Commons. For more information, please contact dcc@fiu.edu. 


\title{
FLORIDA INTERNATIONAL UNIVERSITY \\ Miami, Florida
}

\section{STRUCTURAL FLEXIBILITY AND OXYGEN DIFFUSION PATHWAYS IN MONOMERIC FLUORESCENT PROTEINS}

\author{
A dissertation submitted in partial fulfillment of the \\ requirements for the degree of \\ DOCTOR OF PHILOSOPHY \\ in \\ PHYSICS \\ by
}

Chola K. Regmi 
To: Dean Kenneth G. Furton

College of Arts and Sciences

This dissertation, written by Chola K. Regmi, and entitled Structural Flexibility and Oxygen Diffusion Pathways in Monomeric Fluorescent Proteins, having been approved in respect to style and intellectual content, is referred to you for judgement.

We have read this dissertation and recommend that it be approved.

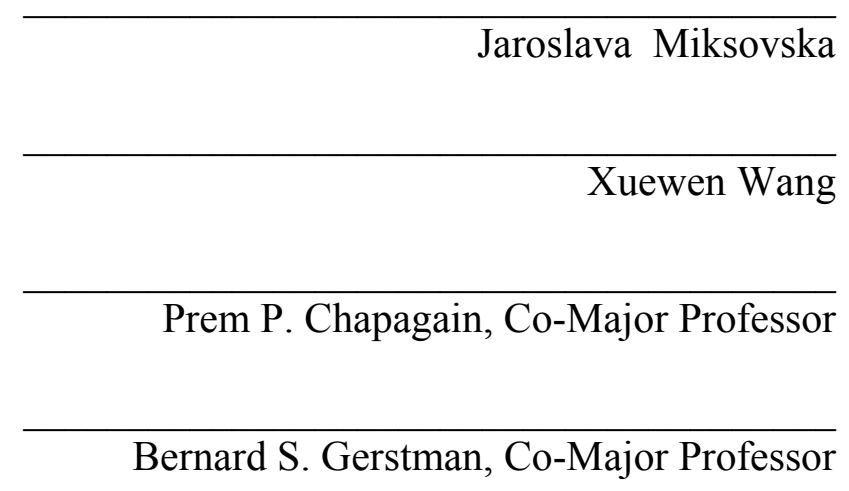

Date of Defense: March 26, 2014

The dissertation of Chola K. Regmi is approved.

$\begin{array}{r}\begin{array}{r}\text { Dean Kenneth G. Furton } \\ \text { College of Arts and Sciences }\end{array} \\ \hline \begin{array}{r}\text { Dean Lakshmi N. Reddi } \\ \text { University Graduate School }\end{array}\end{array}$

Florida International University, 2014 
(C) Copyright 2014 by Chola K. Regmi

All rights reserved. 


\section{DEDICATION}

I dedicate this dissertation to my beloved parents. 


\section{ACKNOWLEDGMENTS}

I would like to express great appreciation to my advisors Professor Bernard Gerstman and Professor Prem Chapagain for their invaluable guidance, help, and support during my entire research work and dissertation writing.

I would like to thank my dissertation committee members Professor Xuewen Wang and Professor Jaroslava Miksovska for their helpful suggestions and comments.

I am thankful to the Biophysics Research Group members Yuba, Jeevan, Tim, and Ilan for their help and cooperation.

I would like to thank my parents, brothers and sisters who always supported and encouraged me towards excellence during the long journey of my education. I am also thankful to my parents-in-law for providing support and care during my graduate studies and research.

Finally, I would like to thank my beloved wife Shailaja for her care, support and understanding throughout these years. Words cannot express my love for my son Aarnes who lived with his grandparents during part of my graduate years when Shailaja was also doing her graduate studies at Ithaca College, NY. 


\title{
ABSTRACT OF THE DISSERTATION \\ STRUCTURAL FLEXIBILITY AND OXYGEN DIFFUSION PATHWAYS IN MONOMERIC FLUORESCENT PROTEINS
}

by

\author{
Chola K. Regmi
}

Florida International University, 2014

Miami, Florida

\section{Professor Prem P. Chapagain, Co-Major Professor \\ Professor Bernard S. Gerstman, Co-Major Professor}

Fluorescent proteins are valuable tools as biochemical markers for studying cellular processes. Red fluorescent proteins (RFPs) are highly desirable for in vivo applications because they absorb and emit light in the red region of the spectrum where cellular autofluorescence is low. The naturally occurring fluorescent proteins with emission peaks in this region of the spectrum occur in dimeric or tetrameric forms. The development of mutant monomeric variants of RFPs has resulted in several novel FPs known as mFruits. Though oxygen is required for maturation of the chromophore, it is known that photobleaching of FPs is oxygen sensitive, and oxygen-free conditions result in improved photostabilities. Therefore, understanding oxygen diffusion pathways in FPs is important for both photostabilites and maturation of the chromophores. We used molecular dynamics calculations to investigate the protein barrel fluctuations in mCherry, which is one of the most useful monomeric mFruit variants, and its GFP homolog citrine. We employed implicit ligand sampling and locally enhanced sampling to determine oxygen pathways from the bulk solvent into the mCherry chromophore in the interior of 
the protein. The pathway contains several oxygen hosting pockets, which were identified by the amino acid residues that form the pocket. We calculated the free-energy of an oxygen molecule at points along the path. We also investigated an RFP variant known to be significantly less photostable than mCherry and find much easier oxygen access in this variant. We showed that oxygen pathways can be blocked or altered, and barrel fluctuations can be reduced by strategic amino acid substitutions. The results provide a better understanding of the mechanism of molecular oxygen access into the fully folded mCherry protein barrel and provide insight into the photobleaching process in these proteins. 


\section{TABLE OF CONTENTS}

CHAPTER

PAGE

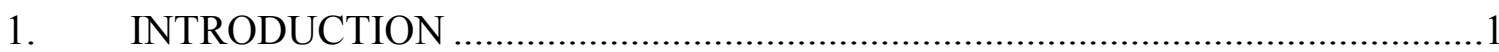

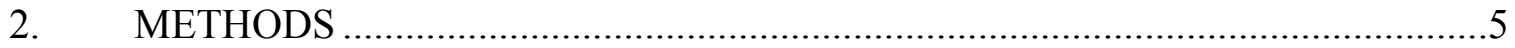

2.1 Molecular Mechanics: Potential Energy Functions .......................................... 5

2.1.1 Bonded Interactions and Bond Related Interactions .................................. 7

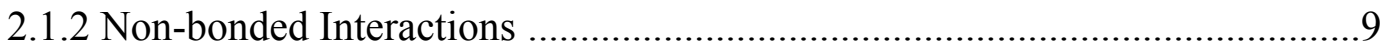

2.2 Molecular Mechanics: Time Integration ..................................................11

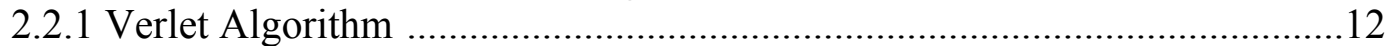

2.2.2 Leap-frog Algorithm ........................................................................... 13

2.2.3 Velocity Verlet Algorithm .................................................................. 14

2.3 Molecular Dynamics: System Setup ......................................................... 14

2.4 Force Field Parameters for Chromophore ................................................19

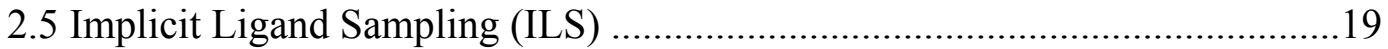

2.6 Locally Enhanced Sampling (LES) .........................................................20

2.7 Simulation Conditions and System Setup ............................................... 21

3. FLUORESCENT PROTEIN BARREL FLEXIBILITY FOR VARIOUS

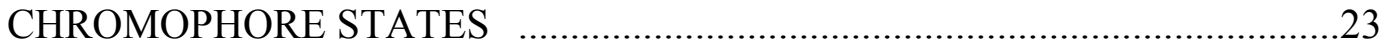

3.1 Fluorescent Protein Structure ........................................................................24

3.2 Different Chemical States of the Chromophore and the Chromophore

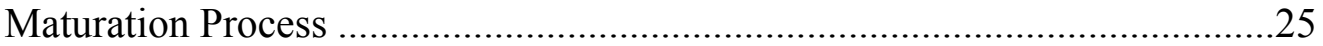

3.2.1 Tripeptide, Neutral, and Anionic States of Chromophore …………….......27

3.3 Root Mean Square Fluctuations (RMSF) of the Protein Barrel and

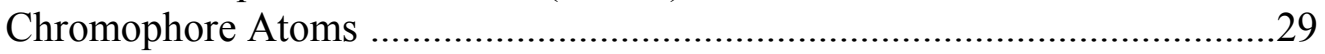

3.4 Investigations of the Gap Between $\beta 7-\beta 10$ Barrel Strands ...............................31

3.5 Correlation Between $\tau$ and $\varphi$ Dihedral Angles ............................................33

4. MOLECULAR OXYGEN DIFFUSION IN mCHERRY AND CITRINE:

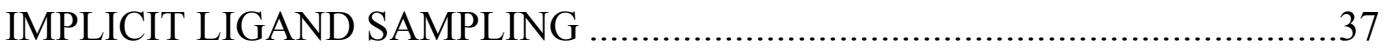

4.1 Implicit Ligand Sampling (ILS) for Molecular Oxygen ................................39

4.2 Importance of Sidechains in Controlling Gap Size .......................................42

4.3 Amino Acid Mutations Made in mCherry to Control Dioxygen Access to the

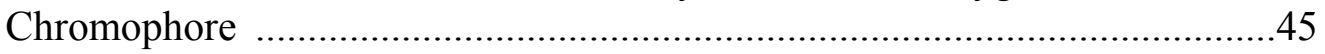

5. MOLECULAR OXYGEN DIFFUSION IN MCHERRY: LOCALLY

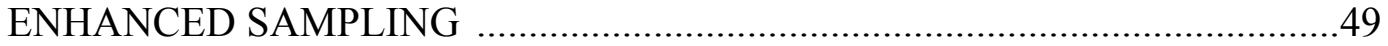

5.1 Molecular Oxygen Diffusion Pathways in mCherry ......................................52

5.2 Free-Energies Along the Pathway Calculated from Implicit Ligand Sampling

5.3 M163Q Mutation and the Enhanced Photostability of mCherry ....................61 


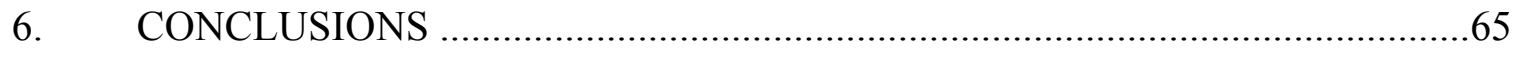

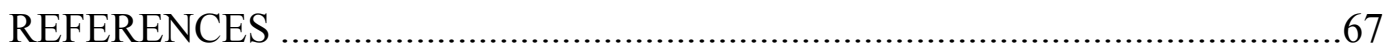

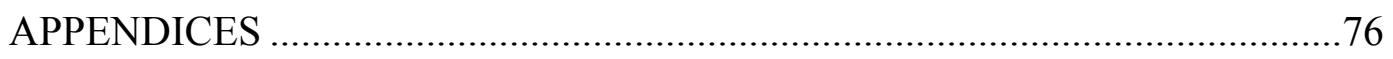

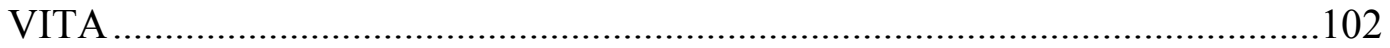




\section{LIST OF FIGURES}

FIGURES

PAGE

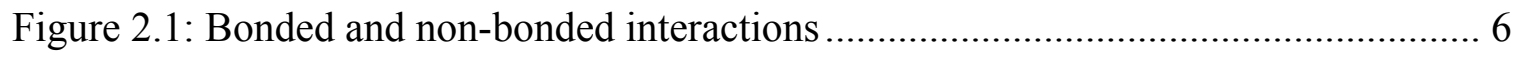

Figure 3.1: Fluorescent protein structure ...........................................................24

Figure 3.2: Chromophore structure of mCherry …...................................................25

Figure 3.3: Chemical mechanism of chromophore maturation ...................................26

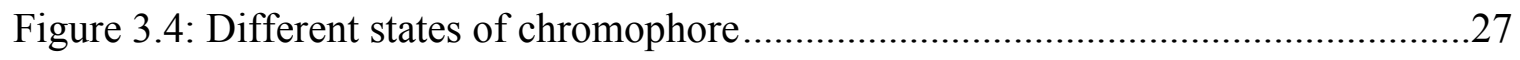

Figure 3.5: Root mean square fluctuations of $\mathrm{C} \alpha$ atoms of protein barrel......................29

Figure 3.6: Root mean square fluctuations of atoms in imidazole ring and phenyl ring of

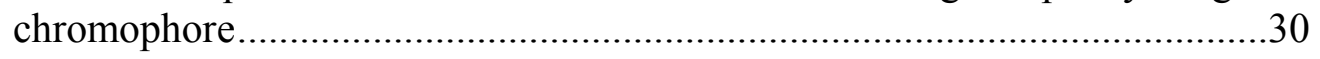

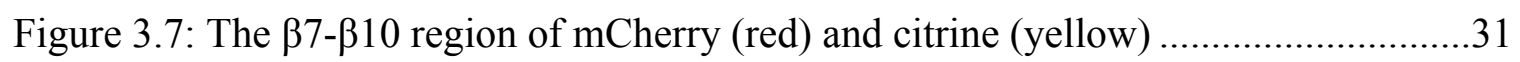

Figure 3.8: Gap fluctuations between $\beta 7-\beta 10$ strands in different chromophore states ....32

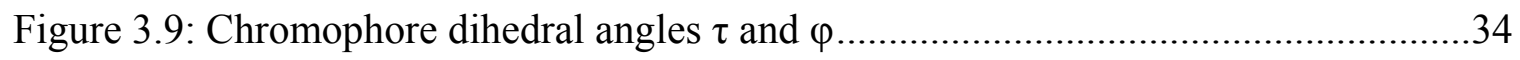

Figure 3.10: Probability distribution for chromophore dihedral angles $\tau$ and $\varphi$ from MD

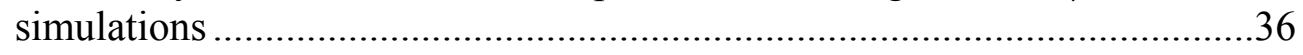

Figure 4.1: Free energy isosurfaces for molecular dioxygen ....................................40

Figure 4.2: Free energy values of dioxygen at locations along the pathways .................41

Figure 4.3: Fluctuations in the $\beta 7-\beta 8$ gap in mCherry ….......................................43

Figure 4.4: Free-energy plot (in $\mathrm{kcal} / \mathrm{mol}$ ) of the $\beta 7-\beta 8$ strands .................................44

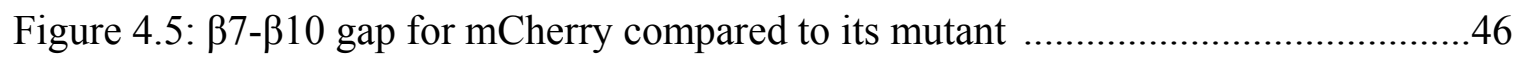

Figure 5.1: Various oxygen-hosting pockets in the mCherry protein barrel ...................53

Figure 5.2: Trajectories of molecular oxygen showing its distance from the chromophore 
Figure 5.3: (a) Volumetric trace of the oxygen diffusion channel from one side of the protein to the other. (b) Amino acid residues involved in the oxygen diffusion channel.....

Figure 5.4: Free-energy values for the oxygen molecule at locations along the curved pathway .60

Figure 5.5: Distribution and schematic of side chain dihedral angles for the gateway residue GLN163 in mCherry versus MET163 in mCherry-Q163M .63 


\begin{tabular}{ll} 
FPs & Fluorescent Proteins \\
RFPs & Red Fluorescent Proteins \\
YFP & Yellow Fluorescent Protein \\
GFP & Green Fluorescent Protein \\
EGFP & Enhanced Green Fluorescent Protein \\
MD & Molecular Dynamics \\
MM & Molecular Mechanics \\
QM & Quantum Mechanics \\
LJ & Lennard-Jones \\
PME & Particle Mesh Ewald \\
PBC & Periodic Boundary Condition \\
VDW & Van der Waals \\
LES & Locally Enhanced Sampling \\
RMSF & Root Mean Square Fluctuations \\
KFP & Kindling Fluorescent Protein \\
FRET & Forster Resonance Energy Transfer \\
ILS & Implicit Ligand Sampling \\
\hline
\end{tabular}




\section{INTRODUCTION}

The development of fluorescent proteins (FPs) has revolutionized cell biology research (1-3). Fluorescent proteins are very useful tools for bio-imaging of molecules, cells, and the bodies of living animals. A green fluorescent protein (GFP) was discovered in the 1960s (4) from the jellyfish Aequorea victoria and the GFP was used as a cell marker in the early 1990s when scientists were able to expressed its gene in other cells (3, 5). Now, these FPs have a wide range of applications in cell biology, such as protein labeling, promoter tracking, timing, Forster resonance energy transfer (FRET), and biosensors $(1,6-8)$.

After the successful expression of the gene for GFP, researchers focused on new and improved variants that are brighter, more photostable, monomeric, faster maturing, and covering a broad spectral range of visible light (8). The S65T (serine to threonine) amino acid mutation in the chromophore of the wild type GFP changed the double excitation peak to a single excitation peak and generated a much brighter variant called the enhanced green fluorescent protein (EGFP) (9). A Y66H mutation on the second residue (tyrosine) of the chromophore of the wild type GFP yielded a blue fluorescent protein (BFP) variant, and Y66W yielded a cyan fluorescent protein (CFP) variant (10). A T203Y mutation in the GFP protein barrel yielded a yellow fluorescent protein (YFP) (11). However, orange and red spectra were not achieved from mutations in the wild type of GFP. The first discovery of a red fluorescent protein (RFP) was from the nonbioluminescent Anthozoa species in 1999 (12), which added more colors to the palettes in cell biology. Recently, Kumagai et al. (6) reported a new class of fluorescent protein, 
UnaG from a Japanese freshwater eel species, which is the first fluorescent protein from vertebrates. The fluorescent mechanism is not same as in GFP, which fluoresces through a chromophore, whereas UnaG fluoresces when it binds with the small molecule bilirubin. The applications of UnaG are not yet fully tested.

In the present work, we focus on the monomeric variants of red fluorescent proteins (mRFPs). They are especially valuable as markers for in vivo applications in mammalian cell imaging (13) because they absorb and emit light in the red region of the spectrum where cellular autofluorescence is low (14). As monomers, the mRFPs are fairly light-weight and therefore do not perturb the processes of the molecules in the cells that are being investigated. In contrast, the naturally occurring fluorescent proteins with emission peaks in this region of the spectrum occur in dimeric or tetrameric forms (12, $15)$, which tend to oligomerize $(16,17)$ and render them unsuitable for fusion tagging (18) because they cause significant perturbations to normal cellular activities. The development of mutant monomeric variants of RFPs to avoid these issues has resulted in several novel monomeric FPs known as mFruits (19). Some of the most promising mFruits are mCherry, mOrange and mStrawberry (20), whose names reflect the wavelengths of their corresponding emission spectra.

Applications of mRFPs would be expanded through the development of variants with higher photostability. Better shielding of the chromophore from the environment to reduce the access of molecular oxygen to the chromophore has been shown to significantly increase the photostabilities of both GFPs and RFPs (21). The Q64H and F99Y mutations introduced in mOrange resulted in the significantly more photostable mOrange2 variant, possibly because these mutations help to block chromophore 
detrimental oxidation by rearranging the structure of the protein-chromophore environment (22). This suggests that irreversible photobleaching can occur as a result of the diffusion of molecular oxygen through the protein barrel surface (16) into the protein interior, in addition to the irreversible photobleaching from transient dark states produced by photoisomerization or excited state proton transfer. Though oxygen access to the mature chromophore can ruin fluorescence, oxygen access is necessary in earlier stages for chromophore formation and maturation. Therefore, understanding of the details of oxygen diffusion pathways in FPs is important from the perspectives of both photostability and chromophore maturation.

The competing effects of oxygen on mFRPs, required for beneficial maturation of the chromophore but detrimental by causing photobleaching, poses limitations to the next generation of single-molecule spectroscopy and low-copy fluorescence microscopy experiments. Therefore, improving the photostability of the mFruits is highly desirable. Increasing evidence suggests that protein flexibility plays a major role in gas access into many proteins (23-28), and dynamic fluctuations in the size of transient cavities due to residues' thermal fluctuations are the determining factor in the pathways of gas diffusion (29-32). For example, oxygen diffusion in myoglobin's distal pocket has been extensively studied, both experimentally and by simulations, in light of the influence of different protein conformations or mutations $(24,33)$. The interaction between the chromophore and the surrounding protein has important implications for both parts of the protein (34). The electronic molecular orbitals of the chromophore that are responsible for its spectral properties may be modified by the surrounding protein and affect the 
spectral properties and the lifetime of the fluorescence (35). Also, the structural fluctuations of the protein may be modified by interactions with the chromophore.

We used molecular dynamics (MD) computer simulations to investigate the dynamic structural behavior of the fluorescent protein barrel in mCherry and citrine, and to determine the role that flexibility of the protein barrel plays in molecular oxygen diffusion through the barrel to the chromophore. Chapter 2 describes the molecular dynamics computer simulation techniques. Chapter 3 describes our results concerning the structural flexibility of the protein barrel in different chromophore maturation states. Chapter 4 describes the oxygen diffusion pathways in mCherry and citrine that we uncovered using implicit ligand sampling computational techniques. Chapter 5 describes the oxygen diffusion pathways using the locally enhanced sampling computational method. 


\section{METHODS}

\subsection{MOLECULAR MECHANICS: POTENTIAL ENERGY FUNCTIONS}

The understanding of the relationship between molecular structure, dynamics, and function in biological macromolecules is greatly facilitated by Molecular Dynamics (MD) simulations (36). Quantum mechanical (QM) treatment of a system gives more detailed properties of a molecular system, and is possibly more accurate than MD, but QM calculations are expensive in terms of computation and not feasible for the systems size of fluorescent proteins. MD simulation, which uses basically classical calculations, is a widely used technique to study the macromolecular properties. Though the results are not as detailed as QM calculations, it is possible to investigate important dynamics of a macromolecule within a reasonable time of computation. Potential energy functions, also called FORCE FIELDS, used in MD calculations are approximations to the exact potential energies experienced by the atoms. The accuracy of the force fields employed compared to the real potentials are critically important in determining the accuracy of the model (37) in simulating to the true behavior of a system.

Numerical values for the parameters used in the force fields are derived using quantum mechanical calculations or obtained by fitting expressions to reproduce experimental results (38) such as high resolution crystal structures.

A well-used MD computational simulation package is CHARMM (37). The CHARMM potential energy function is given in equation 2.1. Bonded interactions between atoms (figure 2.1) are modeled as harmonic potential functions except for the dihedral energy term. Non-bond interactions are also included. 


$$
\begin{gathered}
U(R)=\sum_{\text {bonds }} K_{b}\left(b-b_{0}\right)^{2}+\sum_{\text {angles }} K_{\theta}\left(\theta-\theta_{0}\right)^{2}+\sum_{U B} K_{U B}\left(S-S_{0}\right)^{2}+ \\
\sum_{\text {dihedrals }} K_{\varphi}(1+\cos (n \varphi-\delta))+\sum_{\text {impropers }} K_{\omega}\left(\omega-\omega_{0}\right)^{2}+ \\
\sum_{\text {non-bonded }}\left\{\epsilon\left[\left(\frac{\sigma}{r_{i j}}\right)^{12}-2\left(\frac{\sigma}{r_{i j}}\right)^{6}\right]+\frac{q_{i} q_{j}}{4 \pi \varepsilon_{0} \varepsilon r_{i j}}\right\}+ \\
\text { residuesUCMAP }(\varphi, \psi)
\end{gathered}
$$

where $K_{b}$ is the force constant for bond stretching, $K_{\theta}$ is for bond angle changes, $K_{U B}$ is for the non-bonded Urey-Bradley energy also related to bond-angle bending, $K_{\varphi}$ is for dihedral angle rotations, and $K_{\omega}$ is for improper dihedral angle changes. Similarly $b_{0}, \theta_{0}$, $S_{0}$ and $\omega_{0}$ are the respective equilibrium values. All of these terms are explained in more detail below. Equation 2.1 can be divided into bonded energy terms and non-bonded energy terms. The first four terms and the last term in equation 2.1 are bonded energy terms, whereas the fifth term with brackets contains two non-bonded terms representing van der Waals and electric-charge interactions. The derivative of the potential energy with respect to spatial coordinates provides forces that act on the atoms.

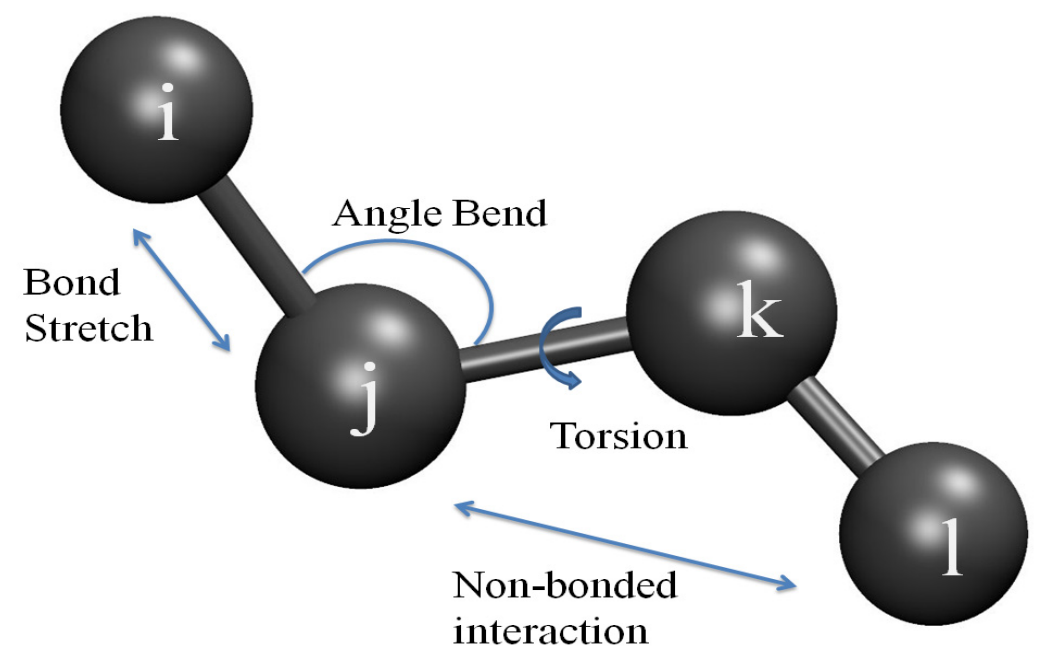

Figure 2.1 Bonded and non-bonded interactions 


\subsubsection{BONDED INTERACTIONS AND BOND RELATED INTERACTIONS}

\section{Bond Stretching Energy}

The first term in equation $2.1, \sum_{\text {bonds }} K_{b}\left(b-b_{0}\right)^{2}$ gives the bond stretching energy between two covalently bonded atoms. The strength of the bond is determined by the force constant $K_{b}$. The value of the equilibrium bond length $b_{0}$ and the force constant $K_{b}$ are specific to the identities of the atoms in the pair. Figure 2.1 shows a system having four atoms i, j, k, and 1. Bond stretching from their equilibrium lengths can occur between pairs of atoms $\mathrm{i}-\mathrm{j}, \mathrm{j}-\mathrm{k}$, and $\mathrm{k}-\mathrm{l}$.

\section{Angle Bending Energy}

The angle bending energy is the energy required to bend an angle formed by two bonds; for example, in figure 2.1 a bond angle can be defined by the pairs $i-j$ and $j-k$. The second term in equation 2.1, $\sum_{\text {angles }} K_{\theta}\left(\theta-\theta_{0}\right)^{2}$ represents the angle bending potential, which also has an harmonic form. $K_{\theta}$ and $\theta_{0}$ are the force constant and equilibrium bond angle, respectively. The bond stretching and angle bending potentials provide the energy necessary to cause a deviation from the equilibrium geometry of the system, which is presumed to have the lowest energy.

\section{Urey-Bradley Energy}

The third term in equation $2.1, \sum_{U B} K_{U B}\left(S-S_{0}\right)^{2}$ is called the Urey-Bradley energy term. This is an energy term that was added in the development of the CHARMM force field to attain better agreement with experimental data on vibrational frequencies. This potential is also represented as a harmonic potential, with force constant $K_{U B}$. The equilibrium distance $S_{0}$ is the distance between the first and third atom in a system of two 
bonds, such as between atoms $\mathrm{i}$ and $\mathrm{k}$ in figure 2.1. It is not included (some $K_{U B}=0$ ) for all $\mathrm{i}, \mathrm{j}$, k bond angles; $K_{U B} \neq 0$ only when the identity of the atoms $\mathrm{i}, \mathrm{j}$, k requires it, along with the improper energy, to optimize the fit to vibrational spectra and out of plane motions.

\section{Torsional Energy (bond rotation)}

The fourth term in equation $2.1, \sum_{\text {dihedrals }} K_{\varphi}(1+\cos (n \varphi-\delta))$ is called the torsional energy of the system, which arises from the rotation about a bond. This potential function comes to play when a rotation occurs around the middle bond of four covalent atoms. For the four bonded atoms $\mathrm{i}-\mathrm{j}-\mathrm{k}-\mathrm{l}$ as shown in figure 2.1 , the angle between the plane $\mathrm{i}-\mathrm{j}-\mathrm{k}$ and $\mathrm{j}-\mathrm{k}-\mathrm{l}$ is called the torsion angle. This potential function is periodic in nature; hence the cosine function. In equation $2.1, K_{\varphi}$ is the constant which gives the strength of the potential and the barrier height resisting full rotation, $\varphi$ is the torsion angle, $\delta$ is an angular phase factor that reflects the most favorable torsional angle, and $n$ is the multiplicity representing the number of potential energy barriers experienced in one full rotation of the angle $\varphi$.

\section{Improper Energy}

The fifth term in equation $2.1, \sum_{\text {impropers }} K_{\omega}\left(\omega-\omega_{0}\right)^{2}$ denotes the improper dihedral angle energy. This energy term is used to maintain planarity or chirality of the system composed of four atoms, $\mathrm{i}-\mathrm{j}-\mathrm{k}-\mathrm{l}$. The improper angle $\omega$ is often defined identically to the torsional angle $\varphi$ for the four atoms, but the form of the potential and the intent are different. The torsional potential described above represents rotations around a bond that may have multiple minimum, whereas the improper term represents a tendency that some systems have to remain planar. Unlike the torsional term, the improper potential term is also defined as an harmonic potential form with force constant $K_{\omega}$. 


\section{CMAP Correction}

The last term in equation 2.1, is $\sum_{\text {residues }} U_{C M A P}(\varphi, \psi)$ called CMAP (correction map) term which is a numerical value of energy added recently to the CHARMM force field to improve the ability to match backbone dihedral angles $\varphi$ and $\psi$ to experimentally observed secondary structure. The latest version of CHARMM, CHARMM36, includes this phi-psi backbone dihedral CMAP correction term, which was not included in earlier versions, such as CHARMM22, and also includes side-chain dihedral angles optimization. This optimization balances the sampling between $\alpha$-helices and $\beta$ regions and corrected the $\alpha$-helical bias of CHARMM22 force fields (39).

\subsubsection{NON-BONDED INTERACTIONS}

The sixth term in equation 2.1, $\sum_{\text {non-bonded }}\left\{\epsilon\left[\left(\frac{\sigma}{r_{i j}}\right)^{12}-2\left(\frac{\sigma}{r_{i j}}\right)^{6}\right]+\frac{q_{i} q_{j}}{4 \pi \varepsilon_{0} \varepsilon r_{i j}}\right\}$ which contains the van der Waals (VDW) interactions term and the electrostatic interaction term, represent the non-bonded interactions in the force field. The van der Waals interactions are represented by a 6-12 Lennard-Jones (LJ) potential and the electrostatic interaction is represented by the Coulomb potential. All atom pairs contribute to the non-bonded energy in the potential function via the VDW interaction.

The repulsive $1 / \mathrm{r}^{12}$ part of the van der Waals interactions can be explained on the basis of the Pauli exclusion principle, and the attractive $1 / \mathrm{r}^{6}$ part is due to induced dipole-dipole interactions. The electrostatic interaction only occurs between atoms with a net charge, $q_{i}$. In equation 2.1, $\sigma$ is the collision diameter between two atoms in the $\mathrm{LJ}$ term, $\epsilon$ is the $\mathrm{LJ}$ potential well depth, and $r_{i j}$ is the intra-atomic distance. In the CHARMM force field the hydrogen bond interactions are accounted through electrostatic and van der Waals 
interactions and therefore there is no explicit energy term for the hydrogen bond interaction in equation 2.1. There are $\mathrm{N}^{2} / 2$ distinct atom pairs to compute the non-bonded interactions in a system of $\mathrm{N}$ atoms. This large number of interactions makes computations of non-bonded interactions in MD simulations more expensive in terms of computational time than bonded interactions. In general, distance cut-offs are used to limit the number of non-bonded interactions that are calculated; atoms separated by a distance greater than this cut-off have their non-bonded interactions set equal to zero without a calculation, thus saving computational time. Since the non-bonded interactions decrease in magnitude as the separation distance $r_{i j}$ increases, a distance cut-off is set large enough, e.g. $12 \AA$, so that atoms separated by this distance have non-bonded interactions that are assumed to be weak enough to ignore.

The empirical potential functions used in classical MD simulations are a compromise between accuracy and efficiency. There are several limitations in the potential functions that give inaccurate calculations of energies and forces in the biomolecular system. One of the major limitations is the setting of fixed charges on the atoms throughout the simulations, which cannot incorporate changes in atomic charges due to reaction mechanisms in a system. In systems where changes in charge are known to be important, more detailed computational approaches are use such as hybrid simulations combining Quantum Mechanical with Molecular Mechanical (QM/MM) models. In the systems that we explore, this is not necessary and our work employs only MM techniques. A crucial aspect of MM techniques is the correct algorithms for the discrete steps through time. 


\subsection{MOLECULAR MECHANICS: TIME INTEGRATION}

Newton's Second Law of Motion is the fundamental principle in MD simulations. Integration with respect to time of the equations of motion gives the time trajectories of the atoms. Discrete time steps are used in MM simulations. The simulations provide the atoms' positions, velocities and accelerations at each time. The acceleration of each particle at each time step is necessary to calculate the next position and velocity of the particles. The acceleration of any particle at a time $t$ can be calculated using $a=\frac{F}{m}$, where $F$ is the force acting on the particle at time $t$ and $m$ is the mass of the particle. The gradient of the potential functions expressed in equation 2.1 gives the force acting on the

particle at a given time through the relation, $F=-\frac{d U}{d r}$. If an initial configuration of a protein is given that contains the $(\mathrm{x}, \mathrm{y}, \mathrm{z})$ coordinates of each atom, then $\mathrm{U}$ can be calculated from equation 2.1 for each particle and then the force experienced by each particle. If the initial velocities of each particle are also provided, then we can determine the future evolution of their positions and velocities with time. Thus, MD simulations are deterministic. In actual MD simulations, velocities are sometimes given random changes, which removes determinacy from the simulations. However, if the random velocity increments are assigned to each particle by following a specific physical distribution, such as the Maxwell-Boltzmann velocity distribution, the dynamics of a protein should be similar from one simulation to the next if both simulations are initialized with the same spatial configuration.

In MD simulations of biomolecules, the initial positions of the atoms can be obtained from x-ray crystallographic structures or NMR structures, and initial velocities 
are randomly chosen using a Maxwell-Boltzmann or Gaussian distributions for the desired temperature. Due to the complex nature of the biomolecules, there is no analytical solution to a Hamiltonian incorporating the potential function of equation 2.1. Instead, numerical algorithms with time increments are used in MD simulations. These integration algorithms are based on the assumption that positions, velocities, and accelerations can be expanded in terms of Taylor series expansions. Below are some commonly used algorithms for performing the numerical, step-by-step time integration.

\subsubsection{VERLET ALGORITHM}

Let $\Delta t$ be the small time step. Then the Taylor series expansion of position as a function of time can be written as:

$$
r(t+\Delta t)=r(t)+\frac{d r}{d t} \Delta t+\frac{1}{2} \frac{d^{2} r}{d t^{2}}(\Delta t)^{2}+\cdots
$$

Similarly,

$$
r(t-\Delta t)=r(t)-\frac{d r}{d t} \Delta t+\frac{1}{2} \frac{d^{2} r}{d t^{2}}(\Delta t)^{2}+\cdots
$$

After addition of the above two equations, we get

$$
r(t+\Delta t)=2 r(t)-r(t-\Delta t)+a(t)(\Delta t)^{2}
$$

in which the first order term in $\Delta t$ does not appear. Equation (2.2) gives the position of a particle at a time $t+\Delta t$, by using the position and acceleration at the current time, $t$, and the position at the previous time, $t-\Delta t$. The advantage of the Verlet algorithm is that it is correct to second order in time $\left(\Delta t^{2}\right)$ but does not require a first order term in $\Delta t$ that depends on velocity explicitly to calculate the new position of the particle. 


\subsubsection{LEAP-FROG ALGORITHM}

In the Leap-Frog algorithm, the position of the particle at time $t+\Delta t$ is calculated by using the relation

$$
r(t+\Delta t)=r(t)+v\left(t+\frac{\Delta t}{2}\right) \Delta t
$$

where the definition for the velocity is

$$
v\left(t+\frac{\Delta t}{2}\right)=\frac{d r\left(t+\frac{\Delta t}{2}\right)}{d t}
$$

The expansion of the velocity in the time step $\frac{\Delta t}{2}$ gives

$$
\begin{aligned}
& v\left(t+\frac{\Delta t}{2}\right)=v(t)+\frac{d v(t)}{d t} \frac{\Delta t}{2}+\frac{1}{2} \frac{d^{2} v(t)}{d t^{2}}\left(\frac{\Delta t}{2}\right)^{2}+\cdots \\
& v\left(t-\frac{\Delta t}{2}\right)=v(t)-\frac{d v(t)}{d t} \frac{\Delta t}{2}+\frac{1}{2} \frac{d^{2} v(t)}{d t^{2}}\left(\frac{\Delta t}{2}\right)^{2}+\cdots
\end{aligned}
$$

Subtraction of these two equations gives

$$
v\left(t+\frac{\Delta t}{2}\right)=v\left(t-\frac{\Delta t}{2}\right)+a(t) \Delta t
$$

where $a(t)=\frac{d v(t)}{d t}$. Equation (2.4) is used to calculate the velocity at time $\left(t+\frac{\Delta t}{2}\right)$ to be inserted in equation 2.3. With this velocity, the position at $(t+\Delta t)$ can be calculated using equation (2.3). The velocities at time $t$ can be approximated by the relationship

$$
v(t)=\frac{1}{2}\left[v\left(t+\frac{\Delta t}{2}\right)+v\left(t-\frac{\Delta t}{2}\right)\right]
$$

The advantage of the Leap-Frog method is that $r(t+\Delta t)$ is calculated more accurately by using $v(t+\Delta t / 2)$ and $a(t)$ than if $v(t)$ is used with $a(t)$. 


\subsubsection{VELOCITY VERLET ALGORITHM}

In the velocity Verlet algorithm, position and velocity at time $t+\Delta t$ can be calculated by using the following relations.

$$
\begin{aligned}
& r(t+\Delta t)=r(t)+v(t) \Delta t+\frac{1}{2} a(t)(\Delta t)^{2}, \\
& v(t+\Delta t)=v(t)+\frac{1}{2}[a(t)+a(t+\Delta t)] \Delta t .
\end{aligned}
$$

In this algorithm, the acceleration at $t$ and at $(t+\Delta t)$ are averaged to calculate positions and velocities. This algorithm is more accurate than the others.

\subsection{MOLECULAR DYNAMICS: SYSTEM SETUP}

In previous sections, we discussed the potential energy function and integration algorithms that are used with Newton's equations of motions to create a trajectory of the motions of the atoms in the molecular system under investigation. As explained above, the computations require an initial $(t=0)$ configuration of the protein molecule containing the $(\mathrm{x}, \mathrm{y}, \mathrm{z})$ positions of all the atoms, as well as the initial velocity of each atom. In this section, we summarize the initialization of the MD simulation. The description provided is based on the CHARMM simulation package.

\section{Initial Structure}

In molecular dynamics we need an initial structure of the system of study to begin simulations. For the simulation of biomolecules, the initial conformation of a molecule can be obtained on-line from the Protein Data Bank (PDB). The structures contained in the PDB are obtained from x-ray crystallography or NMR methods and deposited in the PDB. The structural files obtained from the PDB are in a special format known as a pdb file. In order to use the pdb file for a specific protein in a MD simulation, a careful study 
of the pdb file is required first. Some of the residues or atoms in the protein may be missing in the pdb file. Also, the $\mathrm{x}$-ray structure of a protein contains none of the hydrogen atoms, which means that the hydrogen atoms are not present in the pdb file. The software package MODELLER (40) uses educated guesses from similar sequences in other proteins to add missing residues in the system. The HBUILD command in CHARMM adds the necessary hydrogen atoms to the molecular system.

Once the structure of the molecular system is complete, it may be necessary to change the notation for the names of some atoms to make the pdb file compatible with MD packages. The MMTSB software toolset (41) is a good package for the system setup for use with CHARMM.

\section{Solvation}

Solvation is the process at the beginning of the computational simulation in which biomolecules such as proteins and nucleic acids are surrounded by water to mimic the natural environment of biomolecules. The size of the water solvation box should be sufficiently large to have several layers of molecules outside every part of the protein, including when the protein changes its shape during the MD simulation. This may require a solvation box that is more than twice the length of the initial protein configuration in each direction $(\mathrm{x}, \mathrm{y}, \mathrm{z})$, which can greatly increase the computational time. An alternative method that allows the use of a solvation box that is only a little bigger than the protein is to use periodic boundary conditions (PBC). In this approach, if residues approach the edge of the solvation box, they experience the solvation of water molecules located at the opposite face of the box. In order to prevent residues at one edge of the solvation box from experiencing aphysical interactions with residues on the other side of 
the protein, the size of the solvation water box should be at least the length of the longest axis of the protein plus twice the cutoff distance for non-bonded interactions. This size also usually prevents water molecules at one edge of the box from experiencing interactions with residues from the other side of the protein unless the protein becomes especially elongated.

\section{Neutralization}

Some amino acids have a positive or negative electric charge. Macromolecules may have an overall net positive or negative charge. The Particle Mesh Ewald (PME) (42) method is the technique used to calculate long range electrostatic interactions when PBC is used. To use PME, the system should be overall neutral. In the neutralization step, counter ions are added to make the system neutral. Energy minimization is required after these steps.

\section{Energy Minimization}

After creating a complete pdb file and solvating the protein, it will be possible to start an MD simulation. However, the MD simulation may quickly crash after only a few time steps because the initial positions of the atoms may be physically unrealistic. The positions of the atoms based upon x-ray crystallography, MODELLER insertion of amino acids, and solvation involve guessing. If the initial guesses place atoms too close by even a fraction of an angstrom, the potential energy in some locations in the molecule calculated using equation 2.1 may result in forces and accelerations that are unphysically large. This will lead to unphysically large velocities and gigantic changes in positions that makes the MD simulations numerically unstable, which, for example, might break bonds that, in actuality, do not break. MD simulation packages have built-in tests to determine if 
energies and energy gradients are unphysically large, and to stop (crash) the calculations if the test criteria are violated. To avoid these problems in the MD simulations, MD packages contain routines that move atoms by small amounts relative to their initial positions in order to minimize the molecule's energy. This initial energy minimized configuration may be a local minimum and not the global energy minimum structure. However, this initial energy-minimized configuration is physically realistic and allows the full MD simulation to be performed. The energy minimization steps follow similar procedures as followed during the full MD simulations. However, during energy minimization, the MD program allows unphysically large energies and forces in order to make small rearrangements of the atoms. The PME criteria and potential energy cutoff parameters used during energy minimization should be exactly the same as for the planned MD simulation. There is no definitive number of steps that must be used during energy minimization of a system. Once the change in energy from one time step to the next time step becomes very small $(<0.001 \mathrm{kcal} / \mathrm{mol})$, the system is close to convergence to a local energy minimum and the minimization procedure can be terminated.

\section{Heating and Equilibration}

Once we have a neutral, solvated biomolecular system in its minimum energy configuration we are ready for the next steps before performing the actual MD simulation. If the atoms are assigned velocities according to a Maxwell-Boltzmann distribution corresponding to biologically relevant temperatures $(\sim 300 \mathrm{~K})$, some atoms may be given velocities that are large enough to cause unphysical motions of the atoms that lead to unrealistic changes in configuration. To avoid this, the velocities of the atoms are initially given small values corresponding to low temperature $(<50 \mathrm{~K})$. The system is 
then allowed to perform a certain number of time steps (e.g. 500 or 1000) in order to gently allow the atoms to interact and reach new equilibrium positions. The velocities of the atoms are then given increments corresponding to a small temperature increase (10K or $20 \mathrm{~K}$ ), and the atoms are then allowed to relax again. This process of adding velocity increments to heat the system is repeated until the actual simulation temperature is reached. This incremental heating prevents structural distortion and instability of the system due to sudden large changes of velocity at high temperature. When the system reaches the final temperature, equilibrate is performed until the system properties like pressure, temperature, and energy become stable with time.

\section{Production Run}

The MD simulation that is used to investigate the actual dynamics of the protein is called the "production run". This is the final step of the MD simulation. The production run is performed for a length of time that is set to the expected time for a protein to exhibit an interesting change in configuration. The time evolution of the configuration of the system is called a trajectory which contains the $(x, y, z)$ position and velocity of each atom at each time step. This trajectory can be used to analyze the behavior of the protein. Unfortunately, many structural changes such as folding occur on millisecond or longer time scales that are too long to be feasibly accessible with current computer power. For example, a microsecond of dynamics for a protein with 100 residues may take months to simulate. This limits the type of investigations that can currently be performed with MD simulations. 


\subsection{FORCE FIELD PARAMETERS FOR CHROMOPHORE}

Equation 2.1 for the potential energy requires parameters (force constants and equilibrium bond distances and angles) for every pair of atoms. Most of these parameters are the same for a specified atomic pair in any protein, and the values of these parameters are assigned within the MD package. However, if bonding patterns of a group of atoms is not commonly found, then numerical values for the parameters are required that are

specific to the special arrangement found only in one protein, or a small number of closely related proteins. The numerical values for the parameters for the chromophore for GFP-like fluorescent proteins have been determined (parameterized) by Reuter et al. (43) by using the CHARMM force field parameterization protocol. Parameters for citrine and mCherry chromophores in their neutral and anionic form were adopted from reference 34. For the acylimine part in mCherry, parameters were taken from the CHARMM27 force field.

\subsection{IMPLICIT LIGAND SAMPLING (ILS)}

Implicit ligand sampling (ILS) (44) is a method to find pathways for the diffusion of small gas molecules inside the protein by using MD trajectories that were performed without the gas molecules. ILS is a computational method that computes the potential of mean force (PMF) corresponding to the placement of a given small ligand such as $\mathrm{O}_{2}$ and $\mathrm{CO}$, everywhere inside the protein. The calculated PMF describes the Gibb's free-energy cost of having a particle located at a given position, averaged over all orientations of the ligand at a given position. This free-energy can be determined for many locations in a single time frame of the trajectory, or averaged over all the frames in the trajectory. 
Calculated PMF values also show the area accessible to the ligand for various values of the associated free-energy cost.

The PMF can be estimated by the relation derived in ref. (45);

$$
G(r)=-k_{B} T \ln \sum_{i=1}^{N} \sum_{j-1}^{C} \frac{e^{\Delta E_{i, j}(r) / k_{B} T}}{N C}
$$

Where $G(r)$ is the ligand free-energy when placed at a position $r, C$ is the number of conformations of the ligand, $N$ is the number of MD trajectory frames, and $\Delta E_{i, j}(r)$ is the position dependent interaction energy of the ligand when added at position $r$ to the given frames.

The ILS calculation method can be implemented in the VMD package (46), which uses an MD trajectory as an input file. The protein structure file (psf) should be in XPLOR file format while using VMD to do ILS.

\subsection{LOCALLY ENHANCED SAMPLING (LES)}

In addition to ILS calculations to uncover oxygen diffusion pathways, a locally enhanced sampling (LES) technique (5) was employed. ILS maps out free-energy pathways by placing an oxygen molecule in various static locations in the frames of an MD simulation performed without oxygen. Since the oxygen is placed at fixed locations, the ILS maps out free-energy pathways but does not allow the oxygen to move. Therefore, it is not possible to tell if molecular gates, such as swinging sidechains, may greatly slow the diffusion to the point of being too slow to be of biological relevance. In order to determine if there are biologically relevant pathways for oxygen diffusion, it is necessary to include the oxygen molecule in the MD simulation. However, the important 
pathway may not be where one might expect from examining a frame with a static structure of the protein. Therefore, it is necessary to place many oxygen molecules around the outside of the protein so that, as in a real solution, oxygen molecules can probe the protein at many different locations.

In order to include many oxygen molecules without necessitating many different, time consuming MD simulations, the LES technique is used. In this method, a single oxygen molecule is first placed in the solvated box at a location outside the protein barrel. When the MD production run commences, multiple copies of the oxygen molecule are created, and each oxygen molecule is given a different velocity so that they head in different directions. Within a few time steps, there are oxygen molecules at many locations around the protein surface, interacting with the protein and probing for pathways. Importantly, in this method, one copy of the $\mathrm{O}_{2}$ molecule cannot see or interact with another copy, so they can occupy the same space or pass through each other. This allows a single MD simulation to include multiple oxygen molecules. The psfgen plugin implemented in VMD was used to generate a total of 15 non-interacting copies of the $\mathrm{O}_{2}$ molecule for enhanced searching for diffusion pathways. Simulations were performed with no biasing force or potential that might facilitate oxygen entry into the interior. The NAMD package (47) was used to implement the LES technique in MD simulations.

\subsection{SIMULATION CONDITIONS AND SYSTEM SETUP}

CHARMM and NAMD packages were used for the MD simulations. The MMTSB toolset (41) was used for system setup for the CHARMM package, and VMD was used for the NAMD package. Initial protein structures were obtained from the 
protein data bank (mCherry PDB code: $2 \mathrm{H} 5 \mathrm{Q}$, and citrine PDB code: $1 \mathrm{HUY}$ ). Missing residues were added by using MODELLER (40). The all-atom CHARMM27 force field was used for simulations in both of the packages. The initial structures of mCherry and citrine were separately solvated with TIP3P water molecules with a box cut-off of $10 \AA$ in each of the simulation setups. 


\section{FLUORESCENT PROTEIN BARREL FLEXIBILITY FOR VARIOUS CHROMOPHORE STATES}

In this chapter, we present the results of our work in determining how the flexibility of the protein barrel of two fluorescent proteins (FPs) depends on the chemical state of the chromophore. The reason why this is important is because oxygen must have access to the chromophore in order for the chromophore to undergo the chemical changes that allow it to mature from a tripeptide to the final structure found in FPs. However, after maturation, the protein barrel must act as a shield to prevent oxygen access to the mature chromophore in order to maintain the fluorescent properties. We performed our investigations on one of the most useful monomeric variants of red fluorescent proteins (RFP) mCherry (PDB code: 2H5Q), and also on a yellow variant (YFP) of green fluorescent protein (GFP) citrine (PDB code: 1HUY). Although citrine and mCherry belong to different FP families and the photobleaching mechanisms may be different, we compare the barrel structural integrity of these proteins because of two main reasons. First, citrine is a GFP homologue of mCherry with a similar barrel structure. Second, citrine is the most useful of the FP among all YFPs due to its reduced halide sensitivity and improved photostability $(48,49)$. Other GFPs variants are very sensitive to halides due to easy ion access via a solvent channel or cavity formed close to the dimer interface $(50,51)$ from which other YFPs are derived. In citrine, this cavity is filled by the mutation Gln69Met which prevents the access to the ion (48). A similar effect is desired in mCherry to increase its photostability. We performed MD simulations using these proteins and analyzed the structural dynamics of barrels and chromophores (52). 


\subsection{FLUORESCENT PROTEIN STRUCTURE}

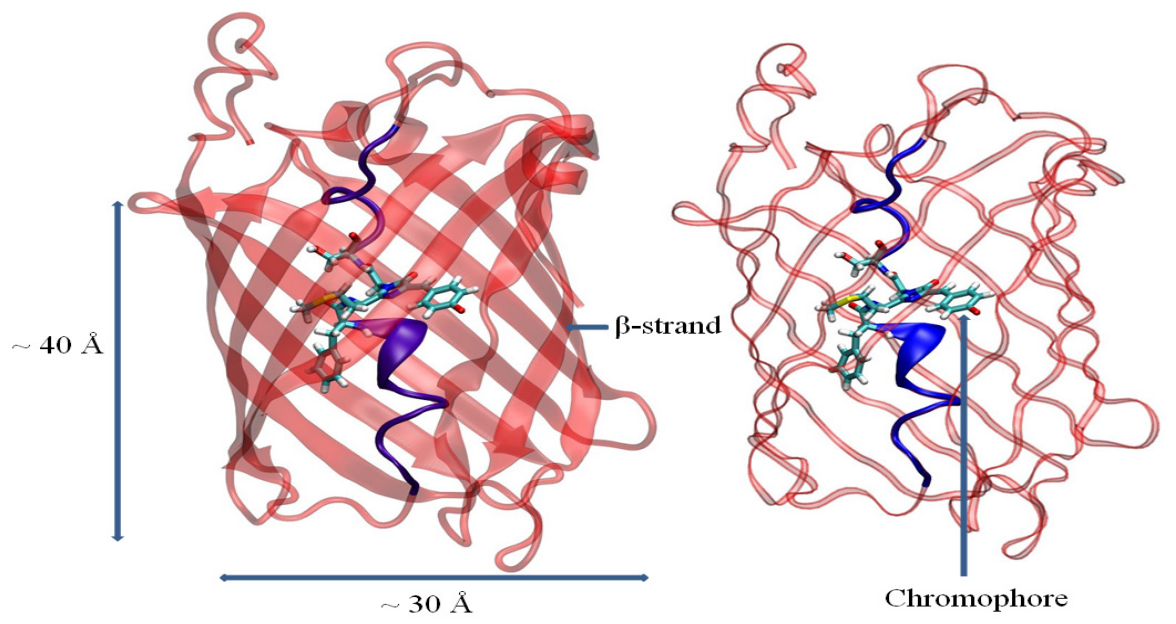

Figure 3.1 Fluorescent protein structure. The chromophore and two connecting residues are shown and the central $\alpha$-helix is colored blue.

Green fluorescent protein (GFP) was first discovered in the early 1960s (4) and its first protein data bank crystal structures (1EMA (11) and 1GFL (53)) were obtained in 1996 by two independent groups. In general, the monomeric variants of fluorescent proteins (FPs) are composed of $11 \beta$-strands which form a cylindrical barrel-like structure (Fig.3.1). Figure 3.1 shows a cartoon-ribbon representation of a fluorescent protein structure. The length and diameter of the cylindrical barrel is $\sim 40 \AA$ and $\sim 30 \AA$, respectively. A central $\alpha$-helix which runs down the geometric center of the $\beta$-barrel holds the chemically modified tripeptide called the chromophore. Figure 3.2 shows a detailed view of the structure of a chromophore. These FPs are formed by $\sim 220$ to 240 amino acids. Loops and short $\alpha$-helices on the top and bottom of the barrel seal the ends of the barrel which protects the chromophore from quenching by restricting oxygen access from the surrounding solvent, and also restricts access of other denaturants (53). 


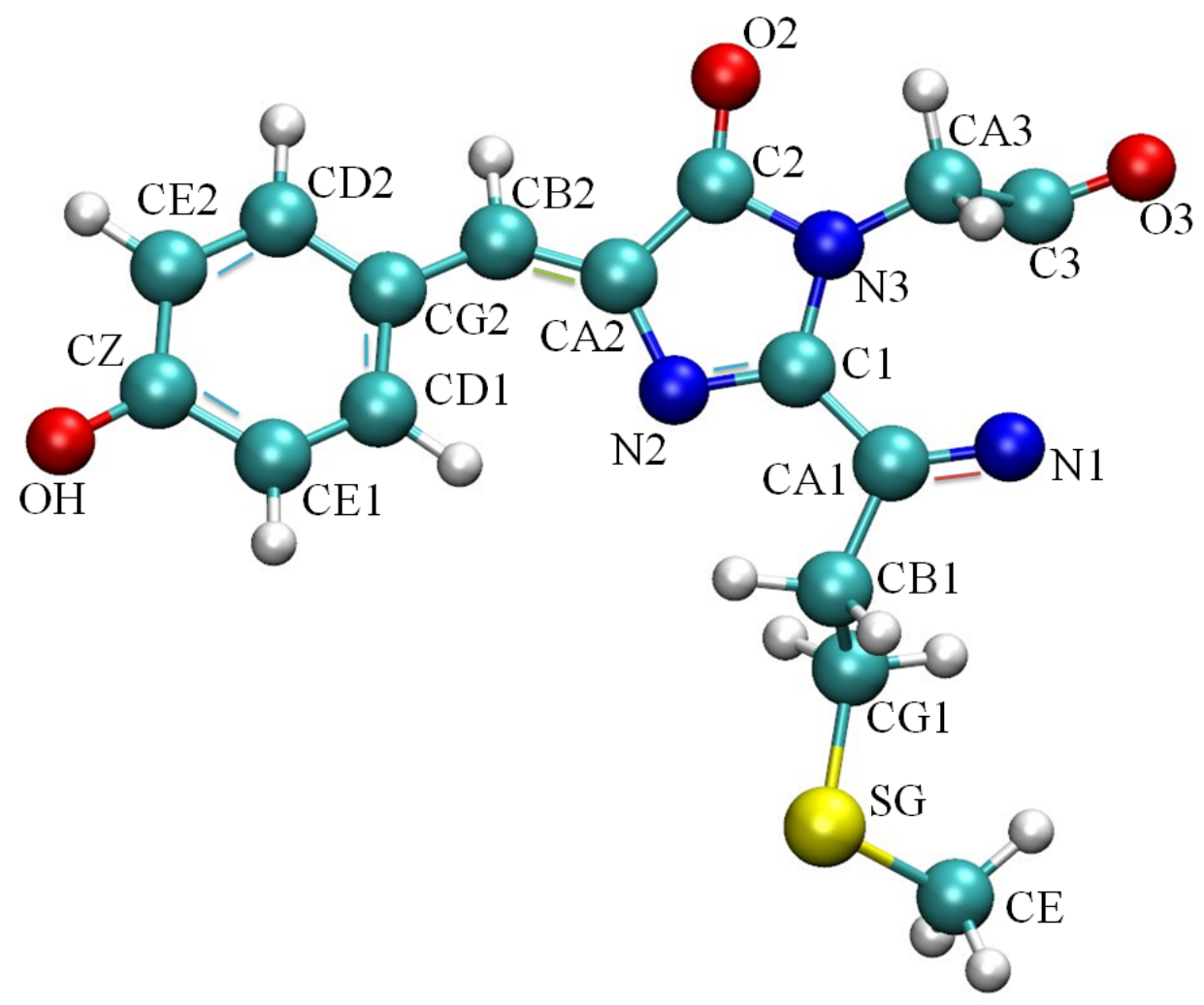

Figure 3.2 Chromophore structure of mCherry. Atoms name are according to pdb file 2H5Q.

\subsection{DIFFERENT CHEMICAL STATES OF THE CHROMOPHORE AND THE CHROMOPHORE MATURATION PROCESS}

In all fluorescent proteins, the chromophore is formed by chemical modification of three amino acids residues at the positions 65-67 (this numbering is based on the wild type GFP). The wild type GFP has Ser-Tyr-Gly as the tripeptide building block for the chromophore. Mutation at position 66 by an aromatic amino acid shifts the emission spectra towards cyne/blue (10). The amino acid residue at position 67 (GLY) is the only conserved amino acid in all fluorescent proteins, and different amino acids can be found at position 65 in different color variants of FPs (54). 


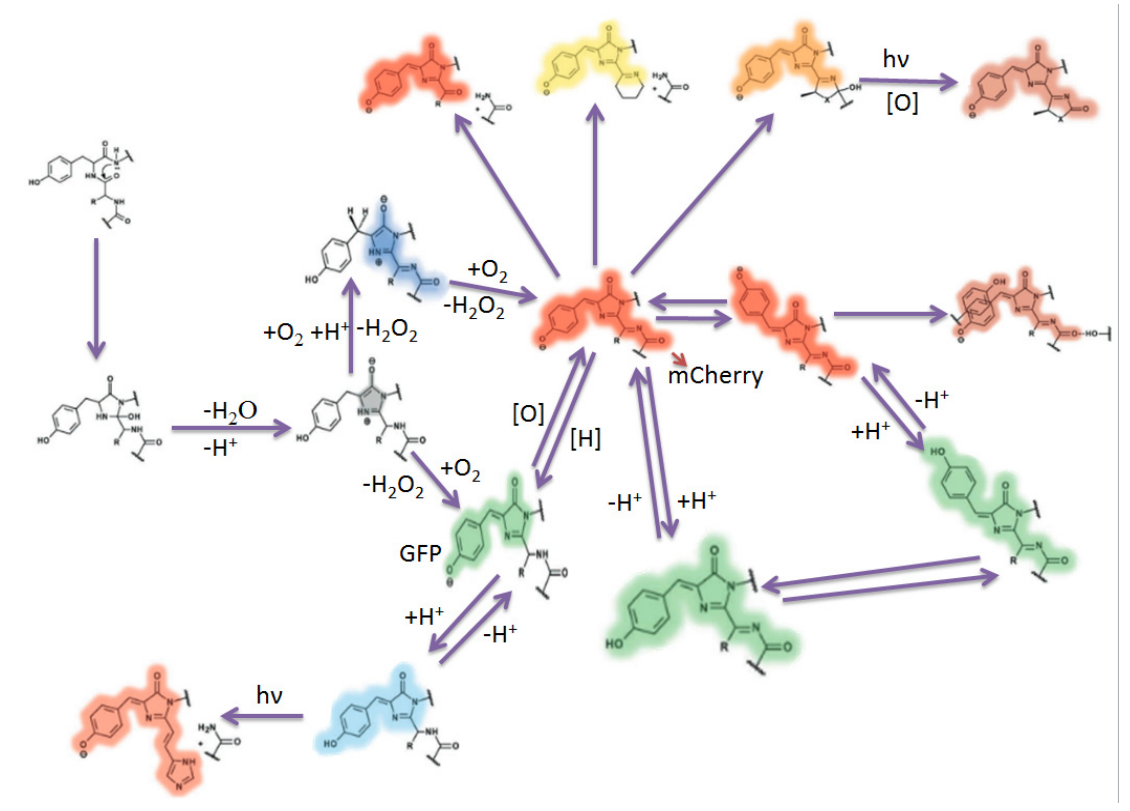

Figure 3.3 Chemical mechanisms of chromophore maturation (adapted from ref. 56). [O] and $[\mathrm{H}]$ represent oxidation and reduction respectively.

The chemical mechanisms of chromophore formation in FPs are cyclization, dehydration, and oxidation. The process starts by nucleophilic attacks on the carboxyl carbon atom at position 65 by the amide nitrogen of glycine at position 67 , followed by dehydration, and results in formation of an imidazolin-5-one heterocyclic ring system. Green fluorescence emission occurs when oxidation of the tyrosine $C_{\alpha}-C_{\beta}$ carbon bond by molecular oxygen extends conjugation of the imidazoline ring system to include the tyrosine phenyl ring. Red fluorescent protein chromophore formation involves a second oxidation step involving the $\mathrm{C}_{\alpha}$ and amide nitrogen at position 65 that further increases the extended $\pi$-bonding electron system to include the carboxyl group of the residue at position 64 (55). A detailed schematic representation of the chromophore maturation steps in GFP and GFP-like fluorescent proteins are shown in fig 3.3 which is adapted from reference (56). 


\subsubsection{TRIPEPTIDE, NEUTRAL AND ANIONIC STATES OF CHROMOPHORE}

The chromophores of citrine and mCherry are formed by the chemical modification of Gly-Tyr-Gly and Met-Tyr-Gly at the position 65, 66, and 67, respectively. The uncyclized three amino acid sequences are referred to as the tripeptide state. The cyclized form of the chromophore which is protonated at its tyrosine phenyl oxygen is called the neutral state, and the deprotonated tyrosine phenyl oxygen form is known as the anionic state. All three chromophore chemical states for each protein are shown in figure 3.4 .

a. Citrine

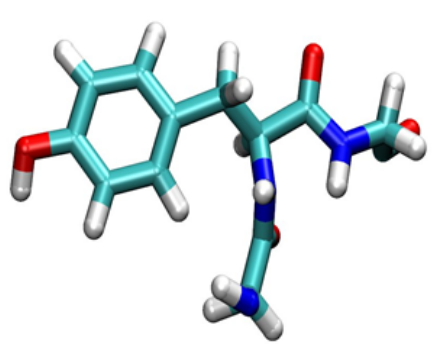

Tripeptide

b. mCherry

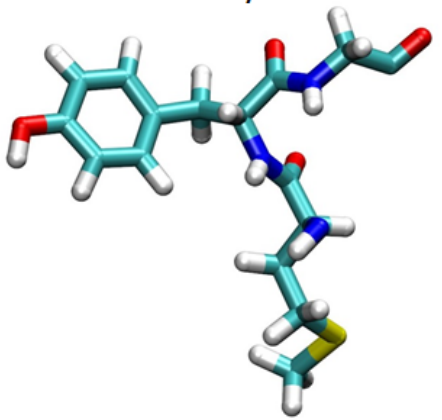

Tripeptide

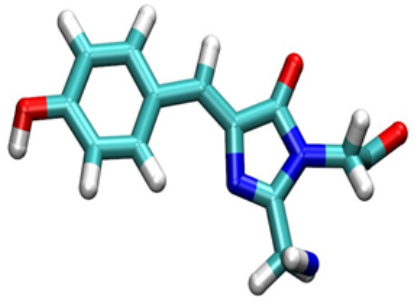

Neutral

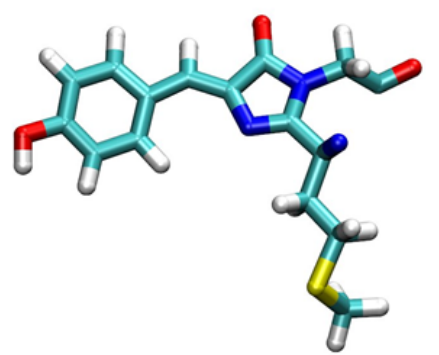

Neutral

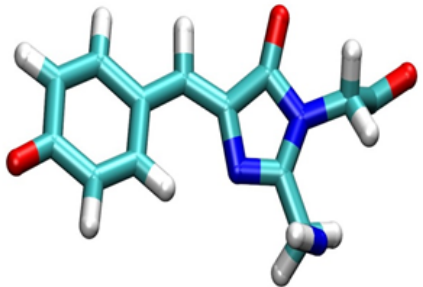

Anionic

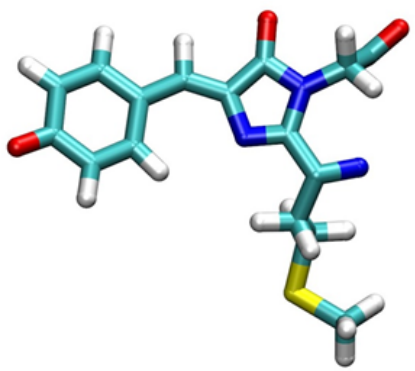

Anionic

Figure 3.4 Different states of chromophore in a) citrine b) mCherry 
We used molecular dynamics (MD) simulation to investigate the structural flexibility of the $\beta$-barrel and chromophore in the different maturation states of the chromophore in citrine and mCherry to determine why oxygen can enter mCherry more easily than citrine. MD simulations of $50 \mathrm{~ns}$ were performed and the last $40 \mathrm{~ns}$ of the MD trajectories are used to analyze the structural dynamics of the protein barrel and the chromophore. In addition, for the deprotonated form of the chromophore at the phenolic oxygen, the protonated Glu222 in citrine and the equivalent residue Glu215 in mCherry were used in their anionic forms.

\section{Methods: MD Setup}

The VMD package was used to setup the protein system for the MD simulations. The initial structures of citrine and mCherry including crystallographic water molecules were solvated by using the solvate plugin in VMD. Using a box cutoff of $10 \AA$, the dimensions of the simulation box were $75.9 \AA \mathrm{x} 73.2 \AA \mathrm{x} 74.3 \AA$ and $83.3 \AA \mathrm{x} 75.6 \AA \mathrm{x}$ $63.4 \AA$ for citrine and mCherry, respectively. The solvated system was electrically neutralized by randomly adding eight $\mathrm{Na}^{+}$ions for citrine and six $\mathrm{Na}^{+}$ions for mCherry in the bulk water using the VMD autoionize plugin. For each system, the Particle Mesh Ewald method was used to treat long-range interactions with a $12 \AA$ nonbonded cutoff. Energy minimization was performed using the conjugate gradient and line search algorithm. The system was then heated with a linear gradient of $20 \mathrm{~K} / \mathrm{ps}$ from 20 to 300 $\mathrm{K}$. At $300 \mathrm{~K}$, the system was equilibrated for $15 \mathrm{ps}$ with a 2 fs integration time step in the NVT (constant number, volume, and temperature) ensemble. Langevin dynamics was used to maintain the temperature at $300 \mathrm{~K}$. The last $40 \mathrm{~ns}$ of the NVT MD simulations with 2 fs time steps was used for analysis. 


\subsection{ROOT MEAN SQUARE FLUCTUATIONS (RMSF) OF THE PROTEIN BARREL AND CHROMOPHORE ATOMS}

Figure 3.5 displays the root mean square fluctuations (RMSF) of the $\mathrm{C}_{\alpha}$ atoms of the protein barrel for the three different states of the chromophore and shows that the overall flexibility of the $\mathrm{C}_{\alpha}$ atoms are almost same in all three states. Loop regions show higher flexibility for the atoms as compared to other regions. In some regions of the barrel, the flexibility of the tripeptide form of the chromophore is less as compared to the neutral and anionic forms of the chromophore. This indicates that the citrine barrel is already rigid even before the chromophore matures. The RMSF analysis of mCherry shows that more flexibility of the protein barrel with the tripeptide state of the chromophore than with the neutral and anionic form of the chromophores. As with citrine, loop regions are more floppy than other parts of the barrel.
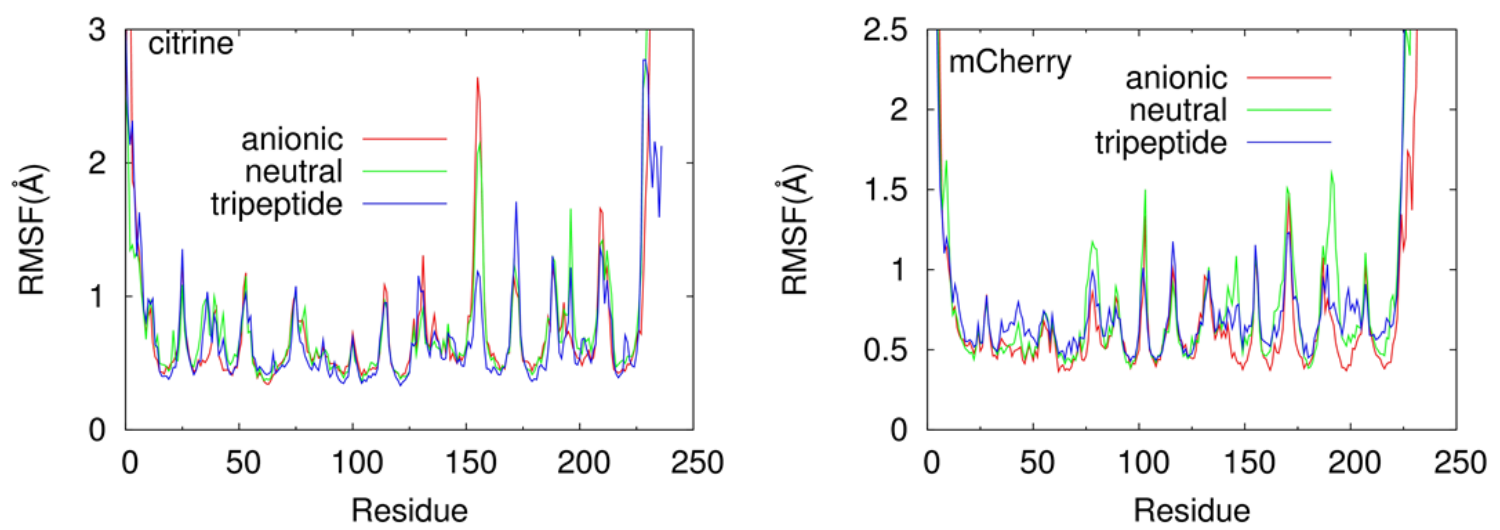

Figure 3.5 Root mean square fluctuations of $\mathrm{C}_{\alpha}$ atoms of protein barrel

Figure 3.6 shows RMSF values for the fluctuations of atoms in the imidazole ring and phenyl ring of the chromophore. The position of these atoms in the chromophore structure is shown in fig. 3.2. The RMSF values show that the atoms in the imidazole ring 

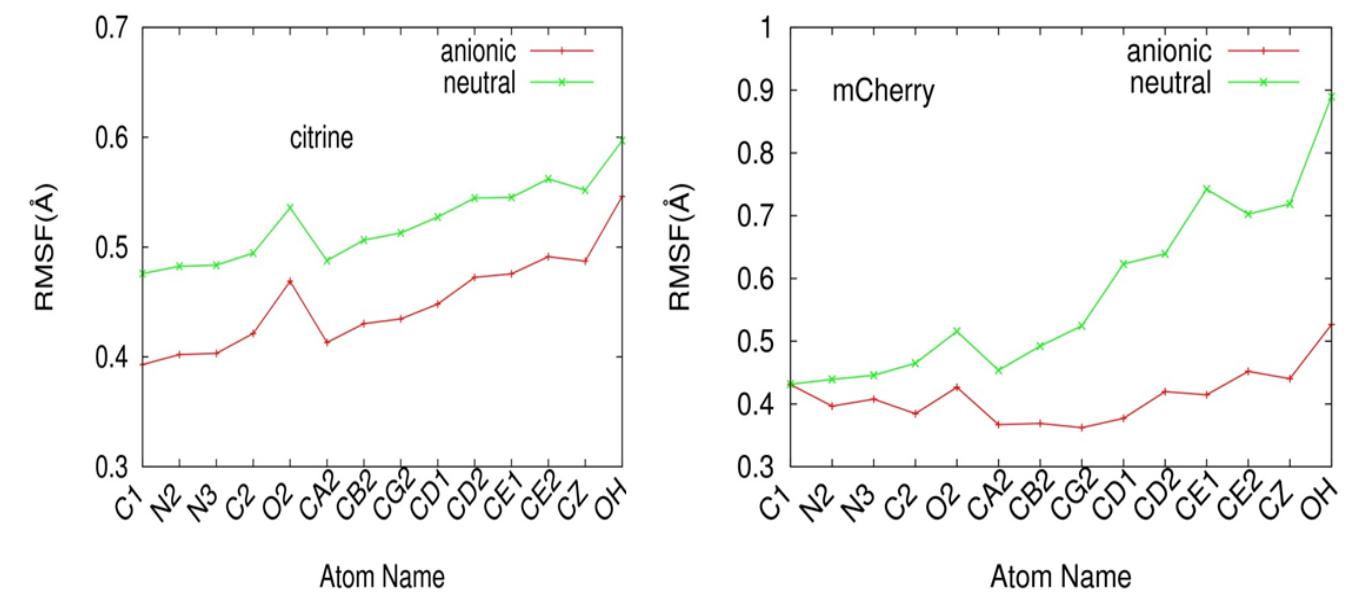

Figure 3.6 Root mean square fluctuations of atoms in imidazole ring and phenyl ring of chromophore. Position of atoms in chromophore structure is shown in fig. 3.2 and phenyl ring in the chromophore are more flexible in the neutral chromophore than in the anionic chromophore in both citrine and mCherry.

The peaks in Fig 3.6 correspond to the atoms in the chromophore which can form hydrogen bonds to the immediate chromophore environment. Flexibility of atoms is observed more in the phenyl ring as compared to the imidazole ring. The phenyl ring is far from the chromophore connection point to the main chain helix. This connection point anchors the chromophore inside the protein barrel. The neutral chromophore, with the protonated phenolic oxygen, makes a weak hydrogen bond with nearby protein residues, which allows increased flexibility of this ring. The rigidity of the chromophore inside the protein barrel is related to the quantum yields because a more rigid structure increases the quantum yield by damping non-radiative modes of de-excitation. The average RMSF of all atoms in the chromophore in the anionic form is $0.46 \AA$ in citrine and $0.50 \AA$ in mCherry and is consistent with the experimental observed quantum yield of 0.76 in citrine (48) and 0.22 in mCherry (19). 


\subsection{INVESTIGATIONS OF THE GAP BETWEEN THE $\beta$ 7- $\beta 10$ BARREL}

\section{STRANDS}
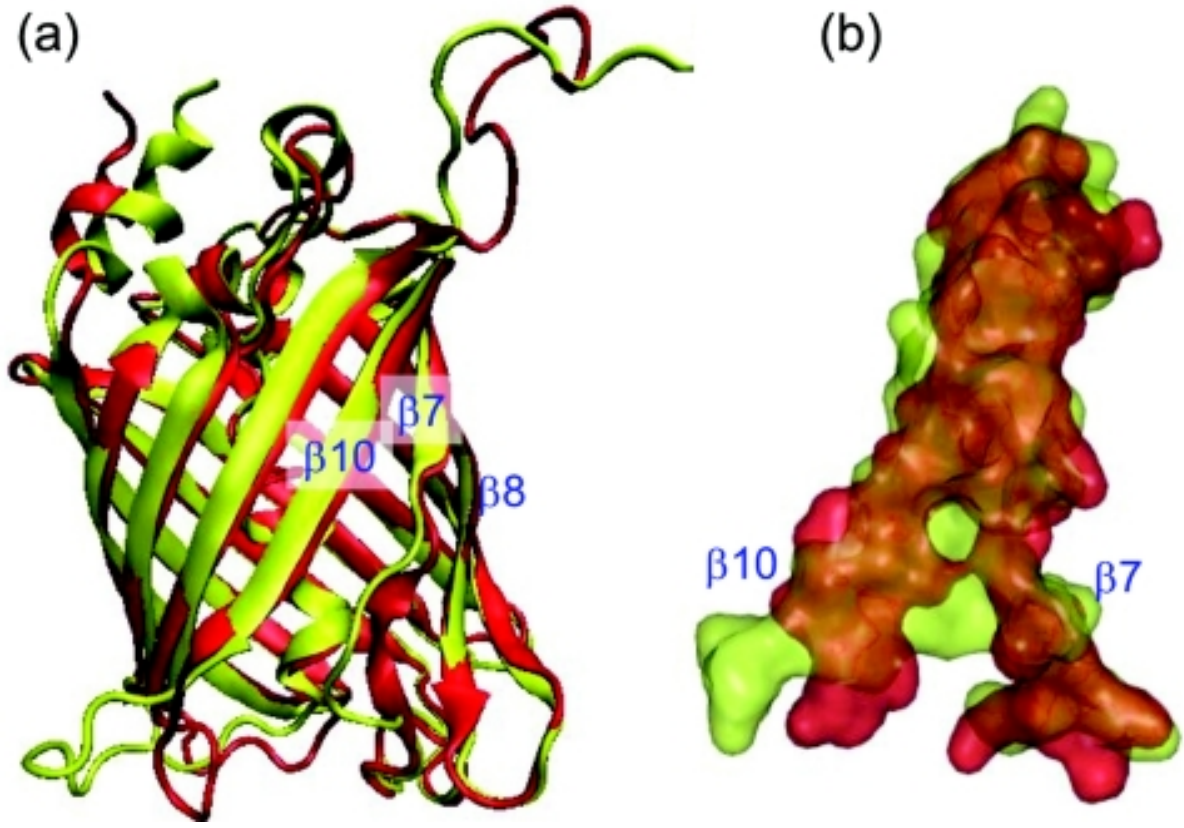

Figure 3.7 The $\beta 7-\beta 10$ region of mCherry (red) and citrine (yellow). a) Superposition of the ribbon structures of mCherry and citrine (52), b) Superposition of space filling structures of mCherry yellow citrine

Dimeric and tetrameric FPs are formed by joining monomers in the $\beta 7-\beta 10$ regions of the protein barrels. Therefore, the $\beta 7-\beta 10$ region is an obvious place to look for structural weakness in the monomeric forms. Figure 3.7 compares the structures of mCherry and citrine. Figure $3.7 \mathrm{a}$ is a superposition of the ribbon structures of the mCherry (red) and its GFP homologue citrine (yellow). Figure 3.7b displays a spacefilling model of the $\beta 7-\beta 10$ region and shows that the gap between $\beta 7$ and $\beta 10$ is smaller in citrine. We investigated the dynamics of this gap in citrine and mCherry using all three states of chromophore. 

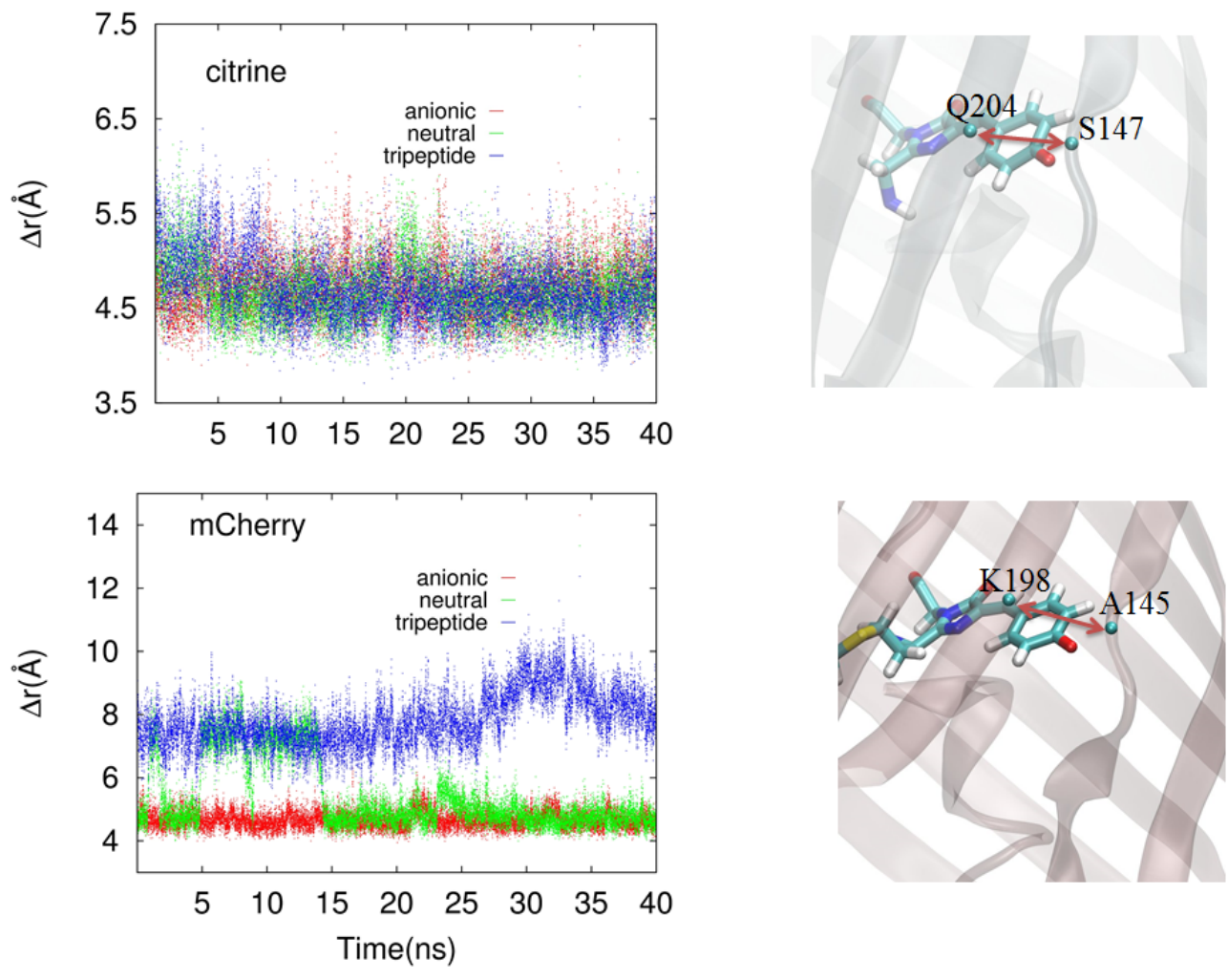

Figure 3.8 Gap fluctuations between $\beta 7-\beta 10$ strands in different chromophore states a) citrine b) mCherry

Figure 3.8 displays time series of the separation between strands $\beta 7$ and $\beta 10$. The plots represent the separation $\Delta \mathrm{r}$ between a pair of residues, one residue on $\beta 7$ and the other on $\beta 10$. In order to characterize the size of the gap, an atom is chosen on each residue that is closest to the other residue across the gap. For citrine, $\Delta \mathrm{r}$ is the distance between the $\mathrm{C}_{\alpha}$ of residues Ser147 and Gln204, and for mCherry, $\Delta \mathrm{r}$ is the distance between the $\mathrm{C}_{\alpha}$ of residues Ala145 and Lys198.

In citrine, the $\beta 7-\beta 10$ gap fluctuations is almost the same in all states of chromophore. The average gap distance from the $40 \mathrm{~ns}$ MD simulations of tripeptide, neutral and anionic states of chromophore are $4.70 \AA, 4.67 \AA$, and $4.70 \AA$, respectively. However, mCherry, which has a slightly bigger $\beta 7-\beta 10$ gap than citrine, shows a larger 
gap opening when the chromophore is in the tripeptide state with an average value of 7.8 $\AA$. In the anionic form of the chromophore the average gap distance of $4.67 \AA$ in mCherry is comparable to the gap in citrine. The neutral form of the chromophore in mCherry has a $5.4 \AA$ average gap that is slightly bigger than citrine.

These analyses show that gap fluctuations between $\beta 7-\beta 10$ is almost the same for all three different states of the chromophore in citrine, but in mCherry the gap flexibility decreases when the chromophore matures from the tripeptide to the neutral and anionic forms. The ability to form a hydrogen bond by the phenolic oxygen of the chromophore with a nearby residue in the $\beta 7$ strand has some effect on gap flexibility. Hydrogen bond calculation shows that in citrine the hydrogen bond between the chromophore phenolic oxygen to His 148 occurs in $38.8 \%$ and $52.4 \%$ of the MD frames when the chromophore is in the neutral and anionic forms, respectively. The equivalent residue Ser146 in mCherry makes a hydrogen bond with the phenolic oxygen of the chromophore only 11.6 $\%$ and $62 \%$ of the time when the chromophores is in the neutral and anionic forms. QM/MM energy calculations of the excited state of the chromophore in the wild-type wtGFP shown that the His148 hydrogen bond with the chromophore phenolate oxygen is sufficient to stabilize the chromophore (57). This shows that the hydrogen bond is important for the stability of the chromophore in fluorescent proteins.

\subsection{CORRELATION BETWEEN $\tau$ AND $\varphi$ DIHEDRAL ANGLES}

Conformational and configurational isomerization can take place when two adjacent bonds twist concertedly (58). This mechanism is called Hula Twist (HT). If the direction of rotation of the $\tau$ and $\varphi$ dihedral angles are in same direction then the correlation of these two angles is positive and called a positive Hula Twist, and if they 
move in opposite direction then the correlation between them is negative and is called negatively Hula Twist (59). The twist around the two rings of the chromophore has been proposed as a radiationless relaxation mechanism of the chromophore from its excited state (7). An NMR study of the fluorescent protein Dronpa also suggested that the fluorescence of the protein depends on the degree of flexibility of the chromophore (60).

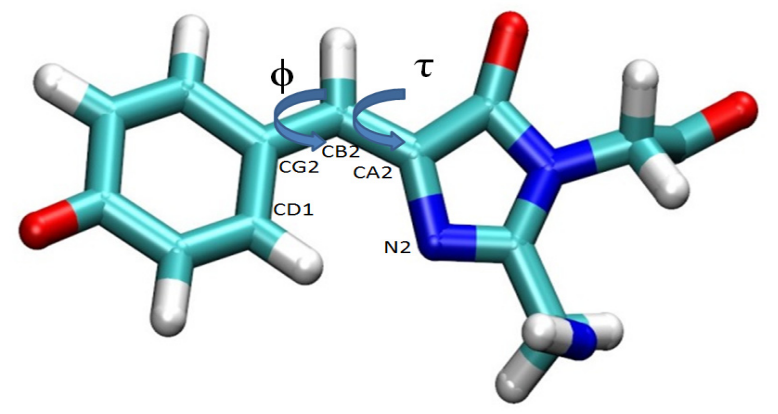

Figure 3.9 Chromophore dihedral angles $\tau$ (N2-CA2-CB2-CG2) and $\varphi$ (CA2-CB2-CG2CD1).

We analyzed the dynamics of two dihedral angles of the chromophore: the tau $(\tau)$ dihedral angle formed by the atoms N2-CA2-CB2-CG2, and the phi ( $\varphi)$ dihedral angle formed by the atoms CA2-CB2-CG2-CD1. These two dihedral angles are adjacent to each other as shown in fig 3.9. There is a double bond between atoms CA2 and CB2 and a single bond between CB2 and CG2 in all GFP and GFP-like fluorescent proteins.

Table 3.1 Dihedral angle (deg) parameters of citrine and mCherry

\begin{tabular}{|l|l|l|l|l|l|l|l|l|l|}
\hline & & $\tau_{\max }$ & $\tau_{\min }$ & $\tau_{\text {avg }}$ & $\phi_{\max }$ & $\phi_{\min }$ & $\phi_{\text {avg }}$ & $\tau_{\text {range }}$ & $\phi_{\text {range }}$ \\
\hline \multirow{3}{*}{ citrine } & anionic & 44.7 & -23.4 & 4.7 & 37.5 & -27.6 & 6.0 & 68.1 & 65.1 \\
\cline { 2 - 10 } & neutral & 22.5 & -27.5 & -0.5 & 36.9 & -36.3 & 3.0 & 50.0 & 73.2 \\
\hline \multirow{2}{*}{ mCherry } & anionic & 40.8 & -26.7 & 6.2 & 36.3 & -38.4 & 4.2 & 67.5 & 74.7 \\
\cline { 2 - 9 } & neutral & 26.6 & -36.0 & 0.6 & 45.8 & -52.0 & -5.7 & 62.6 & 97.8 \\
\hline
\end{tabular}


Table 3.1 summarizes parameters from the MD trajectories for the $\tau$ and $\varphi$ dihedral angles in citrine and mCherry in their neutral and anionic forms of the chromophores. The average $\tau$-dihedral angle in both fluorescent proteins in their neutral form is around $0^{\circ}$ but the anionic forms twist this angle to $\sim 5^{\circ}$. The $\varphi$-dihedral angle analysis shows this angle deviates from $0^{\circ}$ in all situations. This shows that the single bond $\varphi$-dihedral angle conformations are out of planar in these fluorescent proteins. The $\tau_{\text {range }}$ of dihedral conformations in anionic citrine is $18.1^{\circ}$ larger than in the neutral form of citrine, but the $\varphi_{\text {range }}$ is only $8.1^{\circ}$ with the neutral form having the larger range. Similarly, mCherry also has the same trend but the differences in range are $4.9^{\circ}$ and $23.1^{\circ}$. This analysis shows that the neutral form of the mCherry chromophore has more rotational freedom in its $\varphi$-dihedral angle than all other cases. Figure 3.10 shows the probability plots of $\tau-\varphi$ dihedral angles in the neutral and anionic form of the chromophores in citrine and mCherry. These two angles are found to be negatively correlated with a correlation magnitude of 0.53 and 0.49 for anionic and neutral chromophores in citrine, and 0.49 and 0.07 in mCherry.

In conclusion, analysis shows that citrine has a more rigid protein barrel than mCherry. A series of mutations on the tetrameric form of the DsRed fluorescent protein was performed to engineer the monomeric form of mCherry. In this process, mCherry may have lost structural rigidity as compared to citrine which is also an engineered variant from GFP. Flexibility of the chromophore is found to be least in the anionic form of the chromophore as compared to the neutral form in both citrine and mCherry. The hydrogen bond between the chromophore and the barrel residue His148 in citrine and Ser146 in mCherry is found to be critical for stabilizing the chromophore by reducing the 
flexibility. Dihedral angle correlations between the $\tau$-dihedral and $\varphi$-dihedral angles of the chromophore also increase for the chromophore in the anionic form in both of the variants. Cis-trans isomerization of the chromophore have not observed during the MD simulations in any of the cases. The gap between the $\beta 7-\beta 10$ strands seems to be related to the ability to form hydrogen bond between the phenolate oxygen of the chromophore and His148 (in citrine) and Ser146 (in mCherry).
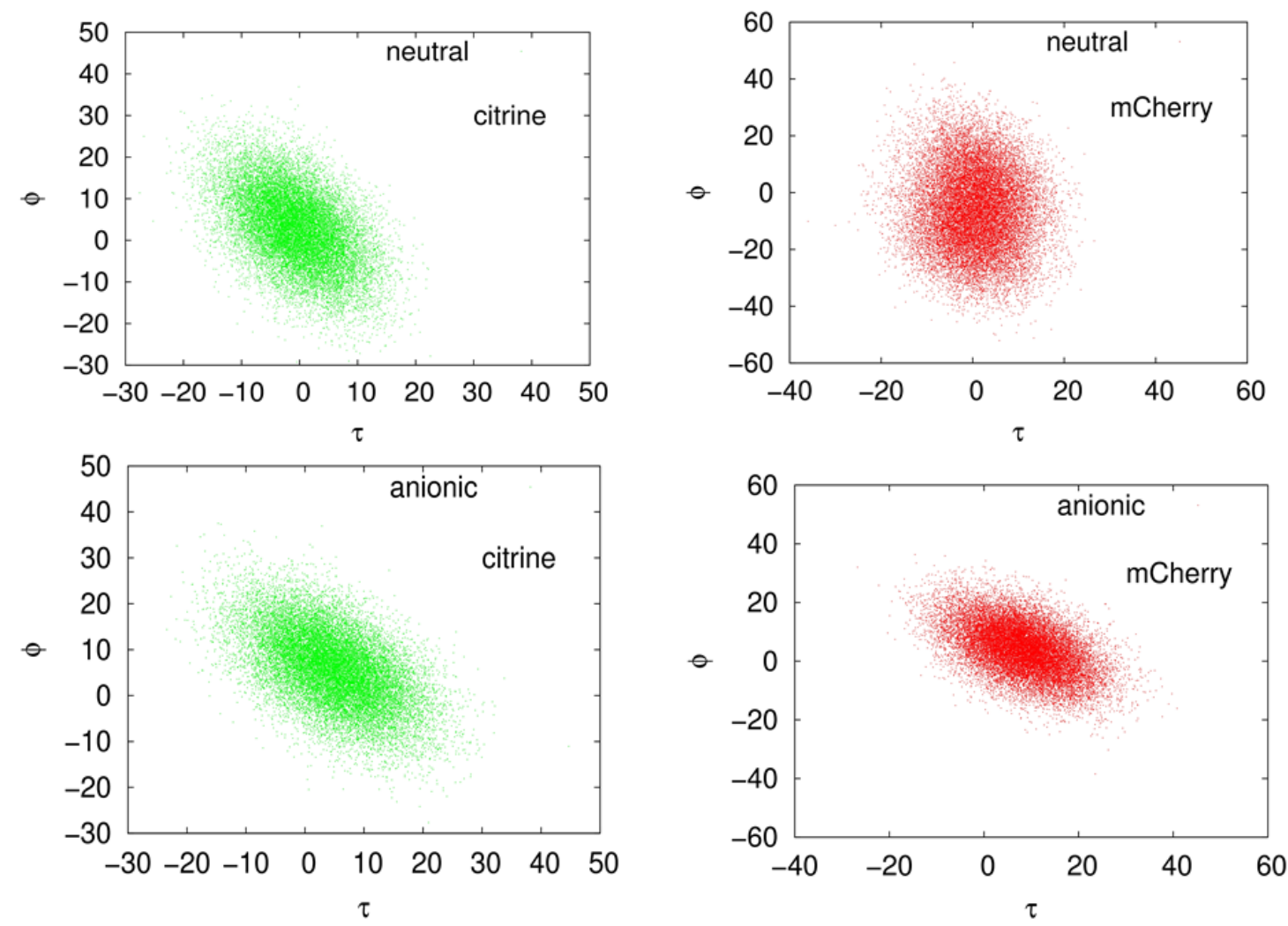

Figure 3.10 Probability distribution for chromophore dihedral angles $\tau$ (N2-CA2-CB2CG2) and $\varphi(C A 2-C B 2-C G 2-C D 1)$ from MD simulation. 


\section{MOLECULAR OXYGEN DIFFUSION IN mCHERRY AND CITRINE: IMPLICIT LIGAND SAMPLING}

Recent developments in efficient computational sampling methods have allowed thorough scanning of the possible pathways for gas diffusion in the interior of interior of proteins (45, 61-64). For example, such computational investigations have proved useful in understanding gas diffusion in may protein systems such as molecular dioxygen pathways via dynamic oxygen access channels in flavoproteins (65-67), ammonia transport in carbamoyl phosphate synthetase (68-70), and gas diffusion and channeling in hemoglobin $(45,71)$. We employ implicit ligand sampling (ILS) to determine oxygen pathways from the bulk solvent into the mCherry active site. Using these results as a guide, we show that the barrel fluctuations and the oxygen pathways can be altered or blocked with strategic amino acid substitutions.

Following the folding of the protein, the chromophore formation involves cyclization of tripeptide and oxidation, which requires molecular oxygen (72). Therefore, the maturation times can depend on the accessibility of molecular oxygen. For example, it is shown that a water-filled pore was essential for fast maturation of TurboGFP chromophore (73). The pore that leads from outside of the barrel to the inside chromophore possibly facilitates molecular oxygen entry. Upon comparing the crystal structures of GFPs and mFruits, structural differences in the beta barrels are observed in Figure 3.7. The tetramer subunit interactions present in the naturally occurring red fluorescent protein DsRed are not present in the mFruit monomeric forms and therefore the latter is expected to have less structural integrity. The crystal structures show larger openings in the mFruits' protein structure, which may be transiently increased further by 
more pronounced thermal fluctuations. These larger openings may allow oxygen to pass more easily to the chromophore which may have implications to both chromophore maturation speed as well as photobleaching due to oxidation.

Figure 3.7a is a superposition of ribbon structures of the RFP mCherry (PDB code 2H5Q) and its GFP homologue citrine (PDB code 1HUY). Figure 3.7b displays a spacefilling model of the $\beta 7-\beta 10$ region and shows that the gap between $\beta 7$ and $\beta 10$ is smaller in citrine. We show that differences in this region create pathways in mCherry for dioxygen diffusion through the barrel to the chromophore.

\section{Methods: MD setup}

The MMTSB toolset was used to set-up the system for simulations. The initial structures of mCherry and citrine were separately solvated in octahedral boxes under periodic boundary conditions with TIP3P water molecules with a box cut-off of $10 \AA$. For mCherry, 11,319 water molecules were used, and for citrine, 9,290 water molecules were used. All water molecules overlapping with the protein were removed. The particle mesh Ewald method(42) was used to treat long range interactions with a 9- $\AA$ non-bonded cutoff. Energy minimization was performed using the adopted basis Newton-Raphson (ABNR) method. Each system was then neutralized by adding sodium counter ions: six sodium ions for RFP and eight sodium ions for YFP. Water molecules that overlapped with the sodium ions were removed and ABNR energy minimization was performed again. The systems were then heated with a linear gradient of $50 \mathrm{~K} / \mathrm{ps}$ from $50 \mathrm{~K}$ to $300 \mathrm{~K}$.

At $300 \mathrm{~K}$, the systems were equilibrated for $2 \mathrm{~ns}$ with a 2 fs integration time step in the NVT (constant number, volume, and temperature) ensemble. The SHAKE algorithm was 
used to constrain the bonds connected with hydrogen atoms. This was followed by a 10 ns NVT dynamics simulation with 2 fs time steps for each protein that was used for analysis.

\subsection{IMPLICIT LIGAND SAMPLING (ILS) FOR MOLECULAR OXYGEN}

We applied PMF/ILS calculations to the frames from our MD simulations to determine locations in a protein that are especially important for blocking, or facilitating oxygen passage, and to quantify the differences at these locations between FP variants. A total of 5,000 protein conformations from a 10-ns MD trajectory were used for ligand sampling. Therefore, the free-energy value at each of the locations is the average obtained from ILS performed every 2 ps for the 10-ns MD simulation trajectory. For the free energy calculation at each location, 20 different rotational orientations of molecular dioxygen were sampled at each gird position with a volume element size of $1 \AA^{3}$. The free-energy is compared to a dioxygen molecule placed outside the protein in the surrounding water, where the free-energy is defined to be zero. In the figures, all locations with a free-energy below $-2.0 \mathrm{kcal} / \mathrm{mol}$ are colored red, and all locations with a free-energy above $+10.0 \mathrm{kcal} / \mathrm{mol}$ are colored blue. The values of the free energy as a function of reaction coordinate were calculated for specific positions separated by

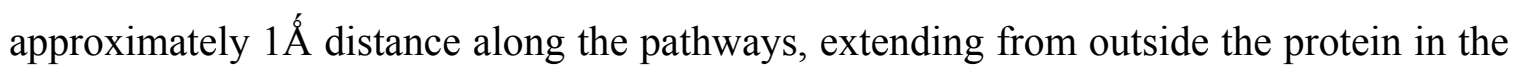
solvent, into the protein and leading to the chromophore. The pathways were determined from visual inspection as well as from the 3D grid data of free-energy values from ILS simulations. 


\section{Dioxygen Access Routes to the Chromophore in mCherry}

The large and fluctuating gap between strands $\beta 7$ and $\beta 10$ displayed in Fig. 3.8 for mCherry makes this region a prime candidate for dioxygen access in mCherry.

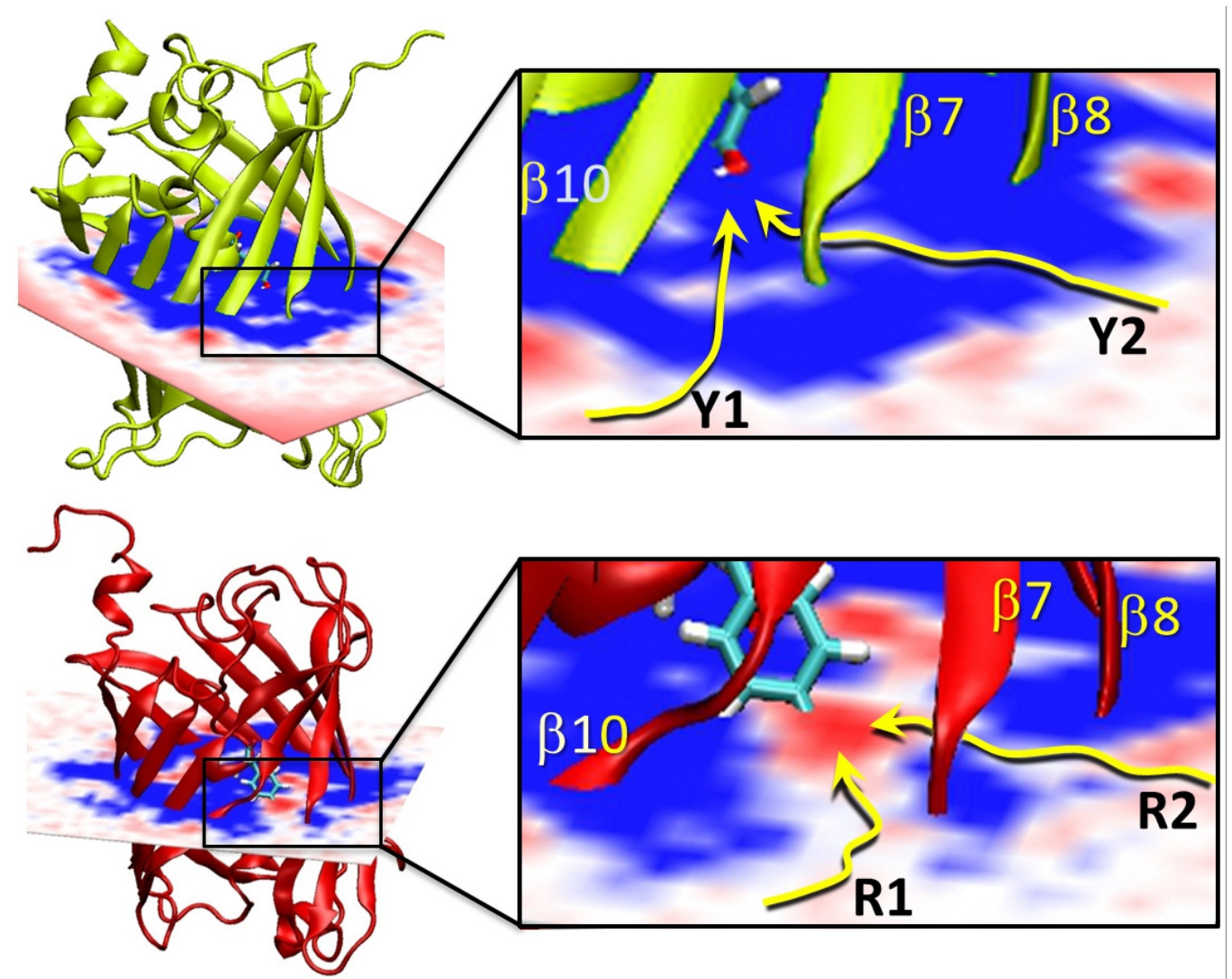

Figure 4.1 Free-energy isosurfaces for molecular dioxygen in (a) citrine and (b) mCherry. The free-energy slice shown includes the $\beta 7-\beta 10$ region as well as the $\beta 7-\beta 8$ region. The color red represents low free-energy ( $<-2 \mathrm{kcal} / \mathrm{mole}$ ) locations, blue represents high freeenergy $(>+10 \mathrm{kcal} / \mathrm{mol})$ locations, and white represents locations for which the oxygen has intermediate free-energy. Neither the $\beta 7-\beta 10$ region nor the $\beta 7-\beta 8$ region in citrine offers low free-energy routes for dioxygen entry, whereas in mCherry both regions display gaps representing low free-energy access routes. 
To determine which regions or pathways allow the molecular oxygen to enter the protein barrel, we calculated the free-energy of placing molecular oxygen in and around the entire protein barrel using ILS, which uses potential of mean force (PMF) calculations to determine the free-energy of placing a small molecule such as dioxygen at a specific location in a protein. Figure 4.1a displays ensemble-averaged free-energy diagrams for a dioxygen molecule if it is placed in, or around, mCherry and compare that with citrine in Fig 4.1b. We calculated the free-energy of the dioxygen using the ILS routine implemented in the VMD molecular dynamics package (46). Fig. 4.1a, display a slice that includes the $\beta 7$ region. The color red represents low free-energy $(<-2 \mathrm{kcal} / \mathrm{mole})$ locations, white represents intermediate free energy locations, and blue represents high free-energy $(>+10 \mathrm{kcal} / \mathrm{mol})$ locations.

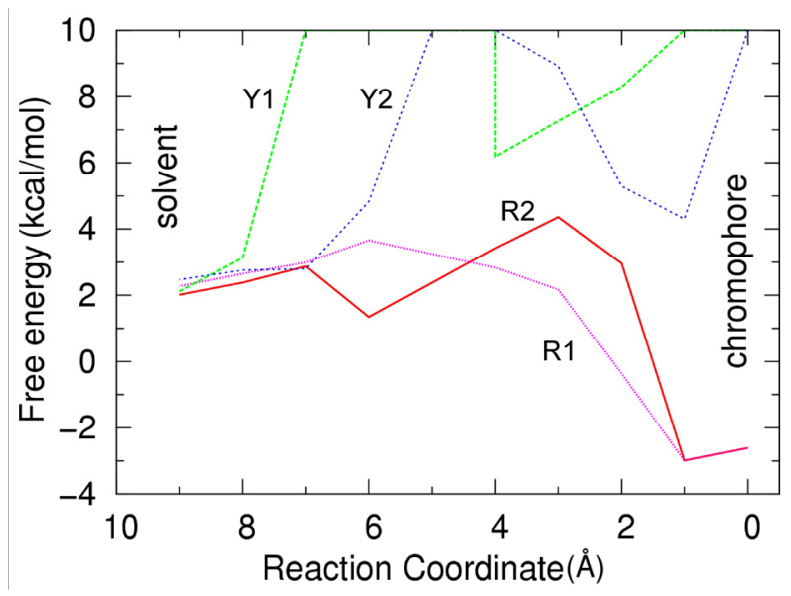

Figure 4.2 Free-energy values of dioxygen at locations along the pathways shown in Fig. 4.1 leading from the solvent outside the protein (9 $⿱$ ) into the chromophore (0 ̊́). The mCherry has two easy routes that can be accessed by entering through either the $\beta 7$ $\beta 10$ gap (R1) or the $\beta 7-\beta 8$ gap (R2). The routes for citrine through either the $\beta 7-\beta 10$ (Y1) region or $\beta 7-\beta 8$ (Y2) region are blocked by a high free-energy barrier. 
In order for the chromophore to have access to molecular oxygen, a pathway without substantial free-energy barriers must exist from the region outside the protein, through the protein barrel, to the chromophore location in the interior. Fig. 4.1a shows that mCherry displays two low free-energy routes through the barrel: one through the $\beta 7$ $\beta 10$ gap (R1) and the other through the $\beta 7-\beta 8$ gap (R2). These two entry routes for dioxygen lead all the way to the chromophore. In contrast, citrine has no easy pathway, including the $\beta 7-\beta 10$ region (Y1) or the $\beta 7-\beta 8$ region (Y2) both of which involve high free-energy (blue) barriers.

Figure 4.2 quantifies the value of the free-energy along the pathways shown in Fig. 4.1. The reaction coordinate is the position of the oxygen molecule along the route. The oxygen molecule is placed at steps along the path that are separated by $1 \AA$. The

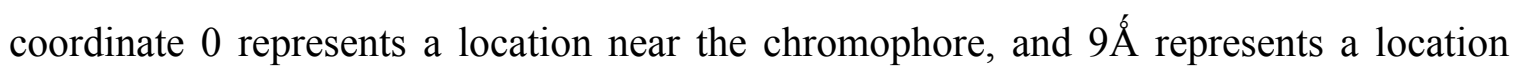
outside the protein in the solvent. It is seen in this figure that both routes in citrine (Y1, Y2) face large free energy barriers due to the protein barrel, whereas there is no substantial barrier for either of the pathways in mCherry (R1, R2). The identification of these pathways is used later to guide mutations of key residues in order to create barriers in mCherry to block these routes and prevent dioxygen access to the chromophore.

\subsection{IMPORTANCE OF SIDECHAINS IN CONTROLLING GAP SIZE}

The information presented in the previous sections show that the $\beta 7-\beta 10$ gap and the $\beta 7-\beta 8$ gap in mCherry are large enough to allow dioxygen to pass through the protein barrel to the chromophore. An aim of this work is to determine if site-specific amino acid mutations can alter these routes. In order to ascertain more details of the structural 
fluctuations in the barrel, we determined if the large fluctuations in the mCherry $\beta 7-\beta 10$ gap is due to motion of strand $\beta 7$ or strand $\beta 10$, and similarly for the $\beta 7$ - $\beta 8$ gap.

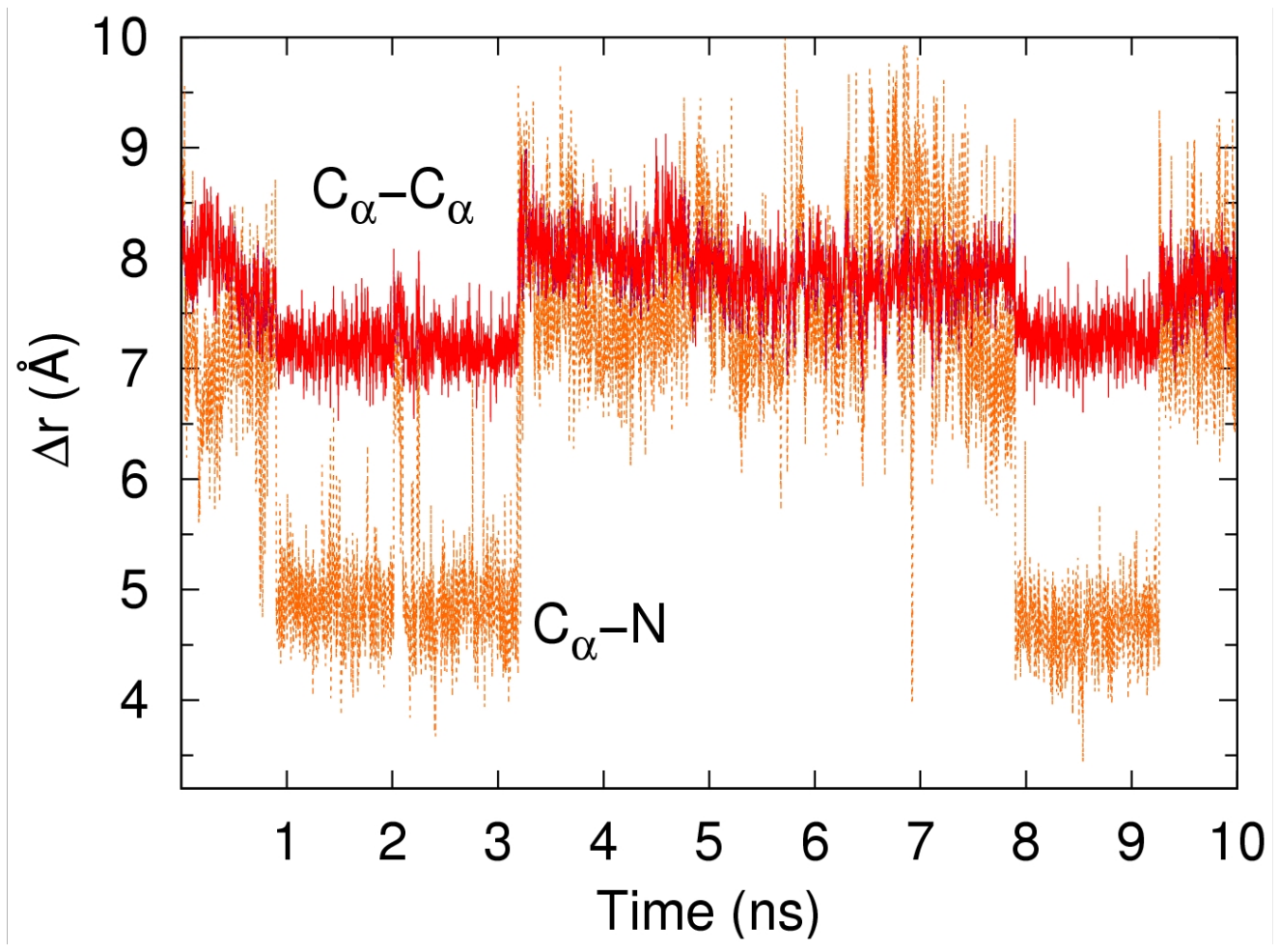

Figure 4.3 Fluctuations in the $\beta 7-\beta 8$ gap in mCherry determined by the distance between $\mathrm{C}_{\alpha}$ atoms of $\beta 7$-Ala145 and $\beta 8$-Gln163 (darker line) compared to the separation determined by the distance between $\mathrm{C}_{\alpha}$ on $\beta 7$-Ala145 and the $\mathrm{N}$ on the sidechain of $\beta 8$ Gln163 (lighter line). The sidechain of Gln163 narrows the gap significantly more than the backbone.

In comparing the time series of the fluctuations in the size of the mCherry $\beta 7-\beta 10$ gap and the $\beta 7$ - $\beta 8$ gap, we found that the openings and closings of the gaps were out of phase with each other. When the $\beta 7-\beta 10$ gap is large, the $\beta 7-\beta 8$ is small, and vice versa, implying that the fluctuations in the $\beta 7-\beta 10$ gap and the $\beta 7-\beta 8$ gap are mostly due to movement of $\beta 7$. In addition, to provide more information for guiding the mutations, we 
wish to determine why Figs. 4.1 and 4.2 both show that the dioxygen pathway through the $\beta 7-\beta 8$ gap (R2) is not as easy as the pathway through the $\beta 7-\beta 10$ gap (R1) even though the backbone separations are similar.

Figure 4.3 show that amino acid sidechains play an important role in closing the $\beta 7-\beta 8$ gap. Figure 4.3 displays the results of $10 \mathrm{~ns}$ MD simulations for the separation between stands $\beta 7$ and $\beta 8$ determined in two different ways. For both time series, the separation is measured between Ala145 on $\beta 7$ and Gln163 on $\beta 8$. One plot shows the time series of fluctuations in the separation between the amino acids' $\mathrm{C}_{\alpha}$ atoms. The other time series displays the fluctuating distance between the $\mathrm{C}_{\alpha}$ of Ala145 (on $\beta 7$ ) and the $\mathrm{N}$ on the sidechain of Gln163 (on $\beta 8$ ). Figure 4.3 shows that the sidechain of Gln163 narrows the gap significantly more than the backbone.

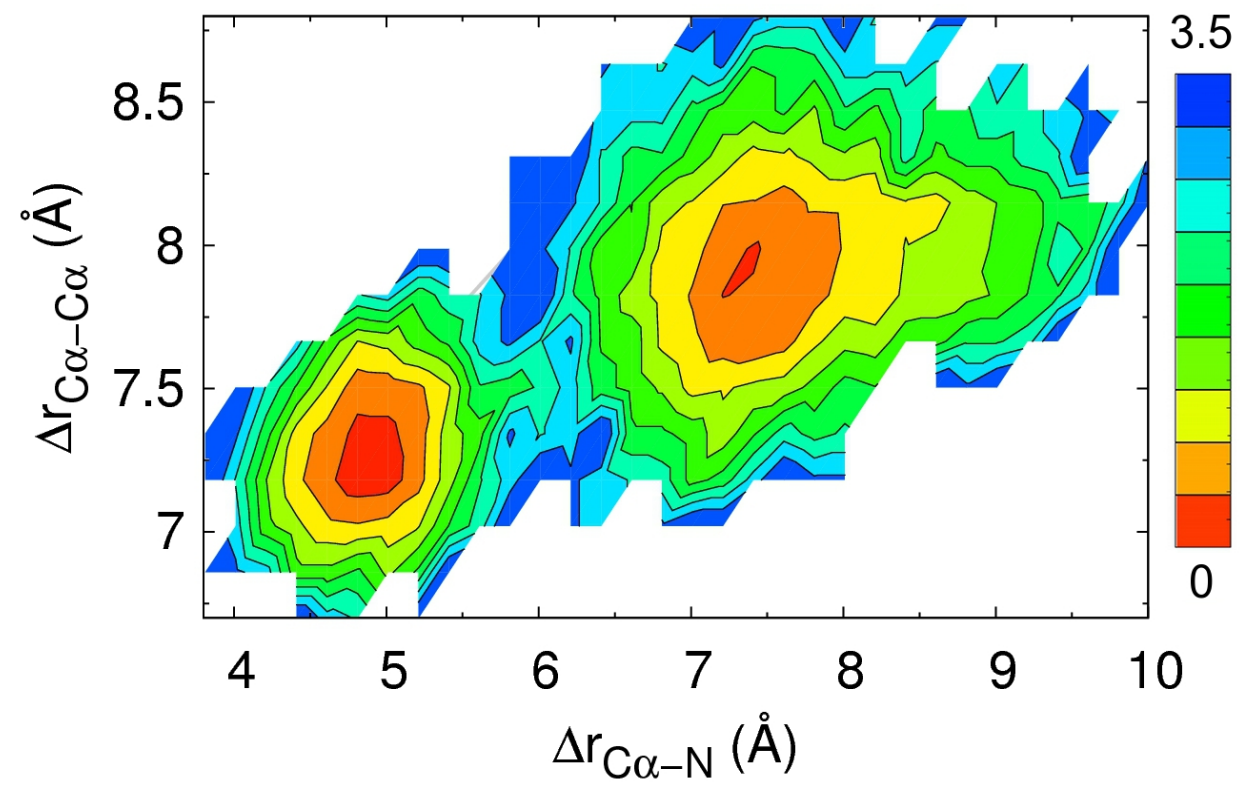

Figure 4.4 Free-energy plot (in $\mathrm{kcal} / \mathrm{mol}$ ) of the $\beta 7-\beta 8$ strands. The horizontal axis is the separation between the $C_{\alpha}$ on $\beta 7$ - Ala145 and the $N$ on the sidechain of $\beta 8$-Gln 163 , the vertical axis is the separation between the $\mathrm{C}_{\alpha}$ of $\beta 7$ - Ala145 and the $\mathrm{C}_{\alpha}$ of $\beta 8$-Gln 163 . 
There are two distinct islands of low free-energy, showing that the $\beta 7$ strand spends most time in these two distinct positions. Additionally, when the $\mathrm{C}_{\alpha^{-}} \mathrm{C}_{\alpha}$ separation is large, the sidechain undergoes larger fluctuations, which restricts the gap from opening widely.

Figure 4.4 further quantifies the importance of sidechains for determining gap sizes. In Fig. 4.4, we present a contour plot of the free energy of Ala145 on $\beta 7$ and Gln163 on $\beta 8$ as a function of their separation, measured in the same two ways that are used in Fig. 4.3. The vertical axis is the separation between the $\mathrm{C}_{\alpha}$ of residue $\beta 7$-Ala145 and the $\mathrm{C}_{\alpha}$ of residue $\beta 8$-Gln163 (dark line in Fig. 4.3) and the horizontal axis is the separation between the $\mathrm{C}_{\alpha}$ of $\beta 7-\mathrm{Ala} 145$ and the $\mathrm{N}$ on the sidechain of residue $\beta 8$-Gln 163 (light line in Fig. 4.3). Figure 4.4 shows that there are two islands of low free energy, which implies that $\beta 7$ is stable at two distinct separations from $\beta 8$, with the larger separation meaning that $\beta 7$ is closer to $\beta 10$. Another important feature is that when $\beta 7$ is further from $\beta 8$, the range of fluctuations in the sidechain of $\beta 8$ are also larger. This allows the large side chain of $\beta 8$ to partially close the gap even when $\beta 7$ is far away.

\subsection{AMINO ACID MUTATIONS MADE IN mCHERRY TO CONTROL DIOXYGEN ACCESS TO THE CHROMOPHORE}

Figures 4.1 and 4.2 show that the easiest pathway for oxygen access in mCherry is through the gap between $\beta 7$ and $\beta 10$, and Figs. 4.3 and 4.4 show that sidechains play a role in closing the $\beta 7-\beta 8$ gap. Therefore, our aim in making amino acid mutations is to decrease the $\beta 7-\beta 10$ gap without substantially increasing the size of the gap between $\beta 7$ and $\beta 8$. 
On comparing amino acid properties of the residues in the $\beta 7, \beta 8$, and $\beta 10$ strands of mCherry and citrine, it is seen that there are more charged residues in mCherry as compared to just two in $\beta 8$ of citrine. The citrine residues in the region of interest are mostly polar (Ser, Tyr, Asn, His) or hydrophobic (Ala, Val, Phe, Ile, Leu).

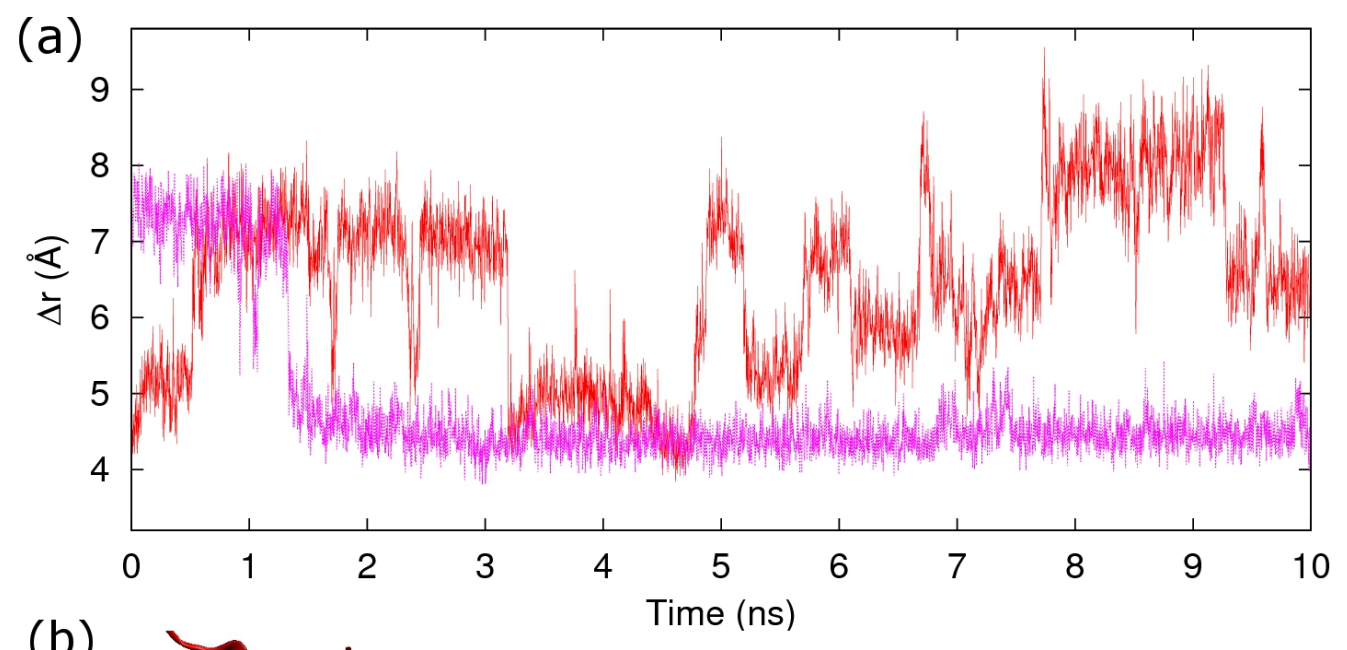

(b)

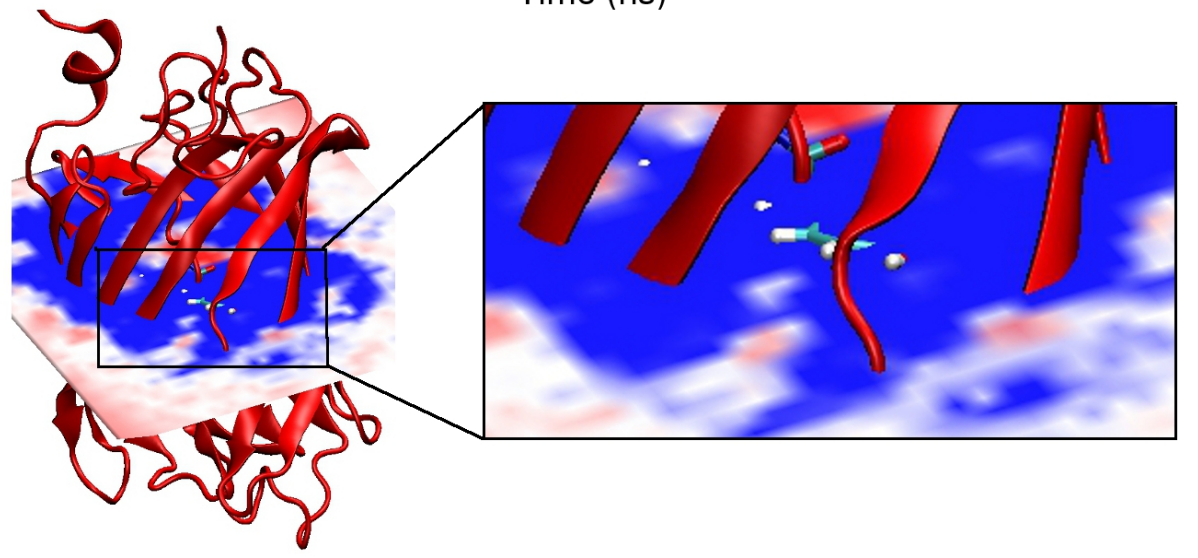

Figure $4.5 \beta 7-\beta 10$ gap for mCherry compared to its mutant (a) Results from the $10 \mathrm{~ns}$ MD simulation of the $\beta 7-\beta 10$ gap for mCherry compared to its mutant (purple). The $\beta 7$ $\beta 10$ gap in mut-RFP is greatly reduced compared to mCherry. (b) Free-energy isosurface for molecular dioxygen in mut-RFP. As compared to the isosurface displayed in Fig. 4.1b for mCherry, mut-RFP isosurface shows significantly less favorable pathways for oxygen entry with high free-energy barriers (blue). 
We first attempted a few mutations in mCherry such that the charged amino acids are replaced by polar or hydrophobic residues, similar to the pattern in citrine. This change, however, either made the fluctuations worse or the $\beta 7-\beta 10$ gap increased even further. Since the $\beta 7-\beta 10$ gap fluctuates the most, a possible strategy to reduce this fluctuation is to create appropriate ionic interactions. In this region of mCherry, the interstrand charged amino acid residues participate in inter-strand ionic interactions and give rise to salt-bridges. For example, the attractive interaction between Glu144(-) in $\beta 7$ and $164 \operatorname{Arg}(+)$ in $\beta 8$ swings $\beta 7$ towards $\beta 8$ and helps to open the gap between $\beta 7-\beta 10$. To reduce the barrel flexibility in this region, two amino acid replacements were made one in $\beta 7$ and one in $\beta 8$. Polar aminoacid $143 \operatorname{Trp}$ was replaced in $\beta 7$ by a positively charged 143Lys(+), and the $164 \operatorname{Arg}(+)$ in $\beta 8$ was replaced by $164 \mathrm{Glu}(-)$. The mutations introduce two possible electrostatic interactions that might close the $\beta 7-\beta 10$ gap. The attraction between the mutated $\beta 7$ 143 $\mathrm{Lys}(+)$ and $\beta 10$ 200ASP(-) pulls $\beta 7$ towards $\beta 10$, and the repulsion between $\beta 7$ Glu144(-) and the mutated $\beta 8$ 164Glu(-) pushes $\beta 7$ away from $\beta 8$ and towards $\beta 10$. Since the location of the gap is close to the original tetramer-breaking mutations, the barrel folding in monomeric form can be sensitive to new mutations such as the one presented here. A new set of mutations must still allow the barrel to fold and chromophore to mature. If this is achieved, the marked reduction in the barrel fluctuations that limit the oxygen entry may result in a more photostable FP.

The success of the mutations in closing the $\beta 7-\beta 10$ gap in the mutated RFP (mutRFP) is shown in Fig.4.5. The two curves display time series for mCherry and mut-RFP for the $\beta 7-\beta 10$ gap. The $\beta 7-\beta 10$ gap in Fig. 4.5 for the mut-RFP starts out at approximately $7.5 \AA$, which is as large as it ever gets for mCherry. This is expected 
because the initial positions for the atoms in our proposed mut-RFP are given by the PDB file for mCherry. Within a short time, Fig. 4.5a shows that new interactions in mut-RFP greatly reduce the $\beta 7-\beta 10$ gap compared to mCherry which significantly reduces the ease of oxygen permeability as displayed by the free-energy isosurface in Fig. 4.5b. 


\section{MOLECULAR OXYGEN DIFFUSION IN mCHERRY: LOCALLY ENHANCED SAMPLING}

Recent investigations have shown that protein flexibility plays a major role in gas access into many proteins (23-28). Conformational flexibility of the side chains of the residues involved in forming transient cavities or pathways can alter the sizes of the bottlenecks for gas diffusion, as well as changing the gating mechanism at the protein surface $(29-32,74)$. In FPs, in addition to affecting the structures of both the chromophore and the protein barrel (34), the chromophore-barrel interaction can also affect the fluctuations of the barrel, which in turn can modify the spectral properties and lifetime of the fluorescence (35). It is shown in a recent important work on cyan fluorescent protein that the reduction in the flexibility of a beta strand in the barrel has led to a dramatic improvement in fluorescence quantum yield (75).

In an important work (76), Roy et al. investigated the diffusion pathways of oxygen in the phototoxic KillerRed protein. In this protein, reactive oxygen is generated from molecular oxygen that diffuses into the interior of the protein. They were able to identify the pores and channels for the oxygen to escape through the protein barrel to the bulk solvent. This study also suggested that the ease of molecular oxygen diffusion through a channel is the cause of the high susceptibility for photobleaching (76). In our work (ref. (52)), oxygen diffusion pathways in mCherry were investigated by implicit ligand sampling techniques which we explained in earlier chapter 4. In that study, an immature tripeptide form of the chromophore was used, and crystallographic water molecules were not included in order to quicken barrel fluctuations so that they could be observed in shorter simulation time scales. To better understand the diffusion process in 
a more realistic setting, we performed molecular dynamics (MD) simulations with explicit molecular oxygen in the system. We use force field parameters for a mature chromophore and also include the crystallographic water in the simulations. The results of these computations describe a pathway that allows oxygen molecules to enter from the solvent and travel through the protein. The pathway contains several oxygen hosting pockets, which are identified by the amino acid residues that form the pocket. We calculate the free-energy of an oxygen molecule at any point along the path. The results provide a better understanding of the mechanism of molecular oxygen access into the fully folded mCherry protein barrel and provide insight into the photobleaching process in these proteins.

\section{Methods: MD setup}

The VMD package was used to setup the system for simulations. The initial structure of mCherry with crystallographic water molecules and one molecular oxygen was solvated by using the solvate plugin in VMD. Using a box cutoff of $10 \AA$, the dimensions of the simulation box were $83.3 \AA$ x $75.6 \AA$ x $63.4 \AA$. The solvated system was electrically neutralized by adding six $\mathrm{Na}^{+}$ions randomly in the bulk water using the VMD autoionize plugin. The final system contained a total of 37,276 atoms. All water molecules overlapping with the protein were removed. The particle mesh Ewald method was used to treat long-range interactions with a $12 \AA$ nonbonded cutoff. Energy minimization was performed using the conjugate gradient and line search algorithm. The system was then heated with a linear gradient of $20 \mathrm{~K} / \mathrm{ps}$ from 20 to $300 \mathrm{~K}$. At $300 \mathrm{~K}$, the system was equilibrated for 15 ps with a 2 fs integration time step in the NVT (constant number, volume, and temperature) ensemble. Langevin dynamics was used to maintain 
the temperature at $300 \mathrm{~K}$. An $80 \mathrm{~ns}$ NVT dynamics simulation with 2 fs time steps was used for analysis.

\section{Structural Features of mCherry}

The first monomeric variant of the red fluorescent protein, mRFP1 was derived from the Discosoma sp. fluorescent protein DsRed $(19,77)$. Development of the monomeric RFP overcame the problems of tetramerization and slow maturation of the parent protein DsRed. However, mRFP1 suffered from lower quantum yield and lower photostability, possibly due to the compromised barrel structure caused by the mutations introduced to break the tetramer interactions at the interface. The monomeric variant mCherry is one of the next-generation monomeric RFPs derived from mRFP1 and has significantly improved photophysical properties (19). Among the mutations introduced to obtain mCherry, Q66M enhanced the maturation, V7I and M182K enhanced the folding, and M163Q removed an unwanted absorbance peak, in addition to significantly enhancing the mCherry photostability (22). As discussed later, our simulation results show that the M163Q mutation in mCherry significantly reduces molecular oxygen entry into the barrel, which might help explain the role of molecular oxygen in permanent photobleaching of FPs and improving the photostability in mCherry.

As with other mFruit variants, the barrel structural integrity is compromised, especially in the $\beta 7$ and $\beta 10$ region, due to missing tetrameric interactions present in the naturally occurring DsRed. Transient thermal fluctuations (52) can allow easier oxygen access to the chromophore. This may help chromophore maturation but can cause fluorescence quenching or faster photobleaching due to oxidation. In cyan fluorescent protein, $\beta 7$ flexibility has been attributed to cause collisional fluorescent quenching due 
to the collision of the Ile146 side chain with the chromophore. In a recent work, structure guided amino acid replacements to reduce $\beta 7$ flexibility have led to a significantly brighter and highly photostable fluorescent protein mTurquoise2, with the highest quantum yield (93\%) among monomeric fluorescent proteins (75).

In ref. (52), the barrel structure of mCherry is compared with that of citrine. Implicit oxygen ligand sampling showed that the $\beta 7-\beta 10$ gap in mCherry provides an easy path for oxygen entry. In addition, static structural comparison also shows differences at the top and the bottom of the barrels. For example, the top of the citrine barrel contains an extra $\alpha$-helix which is not present in mCherry. In this region of mCherry, there is a random coil (or loop segment), and therefore, larger structural fluctuation can be expected. In the earlier study with implicit ligand sampling, no clear entry path for oxygen was observed from the top of the barrel. However, this does not conclusively prove that an oxygen pathway does not exist because implicit ligand sampling may not capture a dynamic pathway that opens and closes but with gatekeeping amino acids that open for a very short time. Moreover, the presence of an actual oxygen molecule can slightly modify the environment in a way that might allow the oxygen to enter the protein barrel. The explicit oxygen simulations carried out in the present investigations are able to capture these possibilities.

\subsection{MOLECULAR OXYGEN DIFFUSION PATHWAYS IN MCHERRY}

For enhanced search statistics, our explicit oxygen calculation employed 15 copies of oxygen in our NAMD LES calculations. The oxygen molecules do not interact with each other, but interact with the rest of the system. With these 15 copies of noninteracting oxygen molecules in the system, an 80 -ns production run was performed. 
The simulations reveal several different types of events of molecular oxygen entry into the protein barrel from various locations, which are pictured in Figure 5.1 and described below. Several protein pockets were observed far from the chromophore where the molecular oxygen can enter and remain for an extended time. Some of these pockets are dead-ends with no access to the chromophore, but some of the pockets join to pathways that ultimately lead to the chromophore (78).

a)

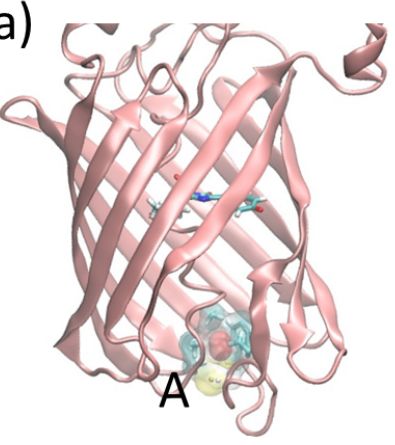

d)

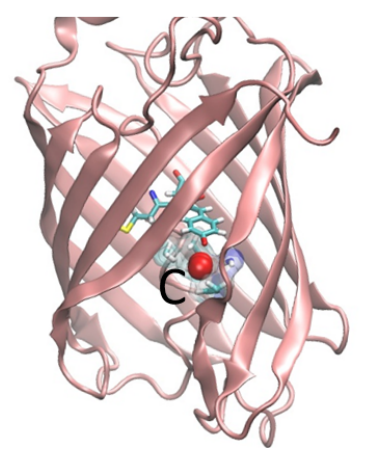

b)

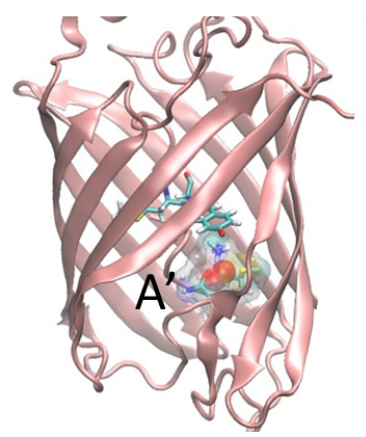

e)

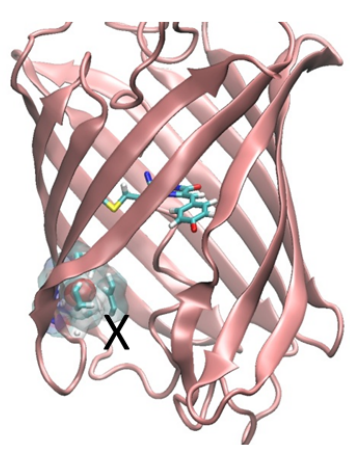

c)

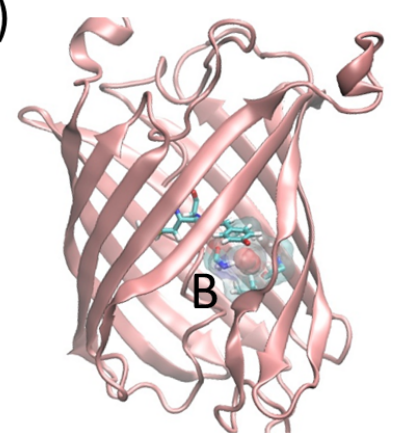

f)

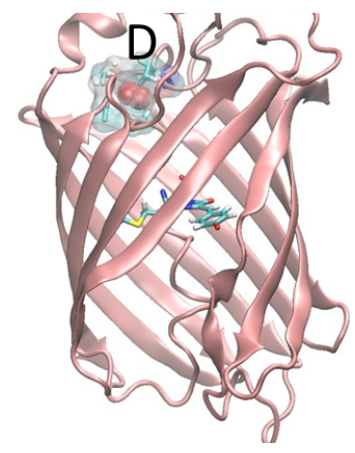

Figure 5.1 Various oxygen-hosting pockets in the mCherry protein barrel. (a-d) Pockets $\mathrm{A}, \mathrm{A}^{\prime}, \mathrm{B}$, and $\mathrm{C}$ are part of the same oxygen diffusion channel. Pocket $\mathrm{C}$ is in the vicinity of the chromophore. Other pockets exist (X and D) but do not connect to the channel. (e) Pocket $\mathrm{X}$ close to the middle of the barrel but off to the side (f) pocket $\mathrm{D}$ near the top of the barrel. 


\section{Diffusion of Oxygen into Pockets Far from the Chromophore}

In our simulations, the first oxygen entry occurred at the bottom of the barrel at approximately $4 \mathrm{~ns}$ into the production run. This oxygen-hosting pocket is formed by the side chains of PHE56, ILE60, PHE129, and MET136. This pocket is labeled as pocket A and displays it in Figure 5.1a. Oxygen enters this pocket through residues HIS25, PRO55, and MET136, which act as gateway residues for multiple entries and exits of the oxygen molecules. It is found that the oxygen can also enter pocket A through residues PRO134 and TYR173. Entry through this gate took $5 \mathrm{~ns}$ to reach pocket A.

We focus our attention on the gate composed of residues HIS25, PRO55, and MET136. The first oxygen that enters at 4 ns escapes back to the solvent after only 200 ps in the pocket. However, another $\mathrm{O}_{2}$ molecule enters again through this gate at $10 \mathrm{~ns}$. This time, the oxygen stays in the pocket for approximately $1 \mathrm{~ns}$ and then moves further into the protein to a second pocket (pocket B) formed by residues GLN64, ARG95, MET97, and VAL105 as shown in Fig.5.1c. Multiple back and forth transitions of the oxygen between pockets A and B were observed. These transitions are shown in Figure 5.2, which displays the distance of the oxygen molecule from a reference point on the chromophore. We chose nitrogen $\mathrm{N} 3$ of the imidazoline ring as the reference point because this part of the chromophore is least flexible. The oscillations of the oxygen (red) between pockets $\mathrm{A}$ and $\mathrm{B}$ occurs rapidly and shows no intermediate pocket. During further simulations, we observed a different oxygen molecule enter pocket $\mathrm{A}$ and also make back and forth transitions between pockets $\mathrm{A}$ and $\mathrm{B}$, but via an intermediate pocket (pocket A' of Figure 1b) formed by residues LEU61, MET97, LEU124, T127, and GLY126. The MET97 side chain forms a part of both pockets A' and B, so the movement 
of this side chain allows the transition between these pockets. Figure 5.2 shows a second trajectory (green) for the oxygen movement that occurs via the intermediary pocket (pocket A'). Ultimately, this oxygen molecule makes the transition to pocket C (Figure 5.1d) and then escapes the protein barrel (Supplementary Movie S1 in ref. 78) as described later. In the $7 \mathrm{~ns}$ time window shown in Figure 5.2, only the second trajectory (green) includes an oxygen transition to pocket $\mathrm{C}$, whereas the first trajectory (red) shows oscillations between pockets A and B only. Supplementary Movie S1 shows approximately the last 4 ns of the green trajectory.

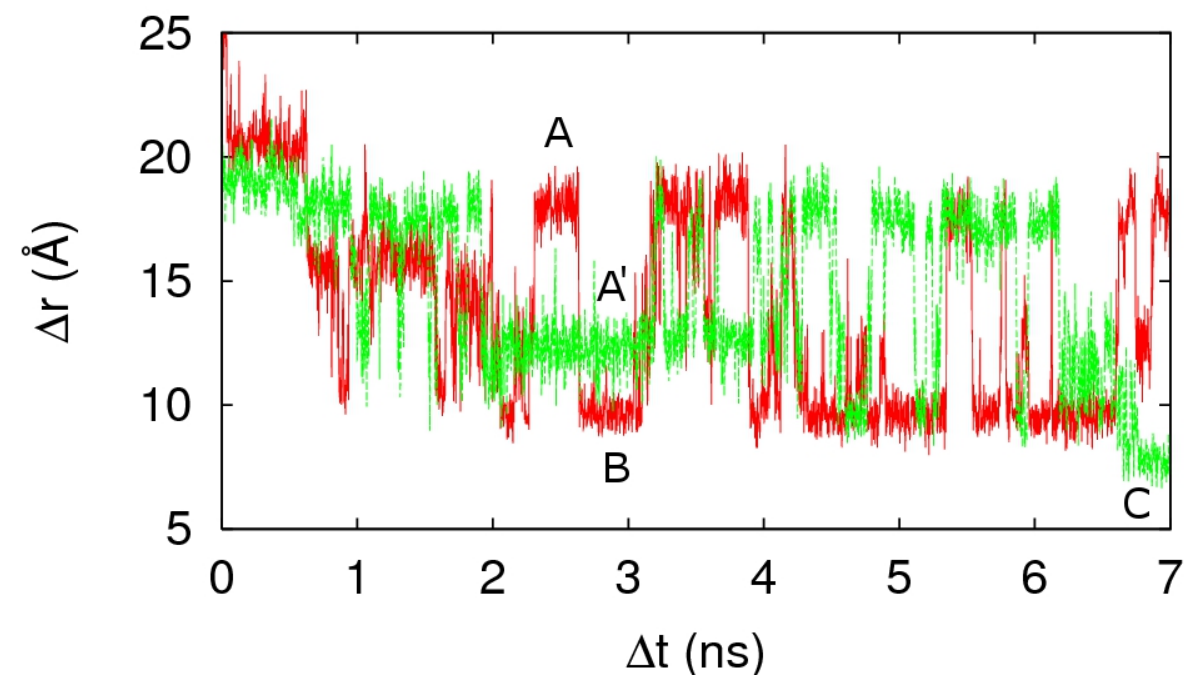

Figure 5.2 Trajectories of molecular oxygen showing its distance from the chromophore for a 7-ns window. One trajectory (red) involves oscillations between two pockets in the protein (pockets A and B of Figure 5.1). The other trajectory (green) involves oscillation between the same two pockets, but also includes oxygen movement in an intermediate pocket, pocket A' of Figure 5.1b, and to pocket $\mathrm{C}$ close to the chromophore. The starting point $\Delta t=0$ corresponds to $52 \mathrm{~ns}$ into the simulation for the green trajectory and $66 \mathrm{~ns}$ for the red trajectory. 
An interesting pocket (pocket $X$ of Figure 5.1e) observed during the simulation is formed by residues LEU46, VAL48, TYR208, THR209, and VAL211, and lies in the barrel interior but off to a side, close to the $\beta 3$ and $\beta 11$ strands of the barrel. This mostly hydrophobic pocket hosts the oxygen for a very long time. Oxygen is observed to enter this pocket at $34 \mathrm{~ns}$ and remain there for the rest of the 80 -ns simulation. Although the oxygen in this pocket is far from the chromophore, $18 \AA$ from the phenolate oxygen, and is not part of any oxygen diffusion channel, we cannot rule out the possibility that an oxygen in this pocket can affect the chromophore, especially since it stays in the pocket for tens of nanoseconds. Another pocket (pocket D, Figure 5.1f) was observed near the top of the barrel and is formed by residues ILE8, PRO37, ALA71, TYR72 and PHE118, with the side chain of TYR72 blocking the oxygen from diffusing further into the barrel. All of these residues are conserved in the mFruits. An oxygen that enters pocket D escapes back to the solvent in less than a nanosecond via the same route that it entered. This pocket may be important because oxygen enters and leaves this pocket multiple times.

We also observed a transient cavity at the barrel surface that is formed by the side chains of LEU54, PHE56, TRP58, and LEU61. These amino acids belong to the central $\alpha$-helix inside the barrel. The oxygen enters this cavity at 9 ns and escapes back to the solvent at $10 \mathrm{~ns}$. No pathways to the chromophore or to other pockets are found, so an oxygen molecule that enters this cavity will quickly escape to the solvent. The side chain of LEU61 separates the chromophore from this cavity and therefore this residue plays a crucial role in preventing the oxygen from reaching the chromophore. Comparison of amino acid sequences shows that these four amino acids are conserved throughout the 
mFruit family, as well as the parent DsRed. Except for LEU54, the other three amino acid residues are also conserved in GFP.

\section{Diffusion of Oxygen into Pockets in the Vicinity of the Chromophore}

The $\beta 7-\beta 10$ region of the barrel is important for oxygen entry because the $\beta 7-\beta 10$ gap undergoes thermal fluctuations that repeatedly produce a large opening. The amino acid residues near this gap, TRP143 of $\beta 7$, GLN163 of $\beta 8$, and LEU199 of $\beta 10$, form a small gateway pocket just inside the surface. This gateway pocket is important because it provides access both to the chromophore and to other pockets further inside the protein, but it is transient, depending on the fluctuations of $\beta 7$ and $\beta 10$. Later in this article, we focus our attention on residue 163 because the side chain of GLN163 is found to be responsible for hindering the diffusion of molecular oxygen further into the barrel. This provides a possible molecular basis for the improved photostability in mCherry, as compared to its predecessor variant, which has MET163 at that position.

In order to investigate the $\beta 7-\beta 10$ gateway pocket in more detail, we cut-out uninteresting computational time during which the oxygen molecule moved around in the solvent outside the protein. The initial entry of an oxygen molecule from the solvent into this gateway pocket required $71 \mathrm{~ns}$. In order to avoid the computational wait-time for the oxygen in the solvent to get to the gate point between $\beta 7-\beta 10,20$ new simulations were

run. Instead of LES with 15 different oxygen molecules, we used 20 independent simulations, each with just one oxygen molecule placed at the $\beta 7-\beta 10$ gate. This expedited the search for pathways into the protein through the $\beta 7-\beta 10$ gate. A simulation was terminated if the oxygen molecule escaped the protein and went out to the solvent. We considered the oxygen molecule to have completely escaped and terminated the 
simulation run if the oxygen's distance from the chromophore's phenolate oxygen exceeded $15 \AA$. This distance ensures that a simulation will not be terminated if the oxygen has not truly escaped the $\beta 7-\beta 10$ gate and an oxygen that remains in the vicinity just outside the pocket will be given time to reenter. In nine runs, the oxygen molecule escaped immediately (within picoseconds). In the other 11 of these 20 simulations, the oxygen molecule entered completely into the gateway pocket. For 10 of these 11 simulations, the oxygen molecule remained in the pocket for $13 \mathrm{~ns}$ on average, before escaping back into the solvent. Only in one simulation did the oxygen molecule manage to diffuse further into another pocket, pocket C shown in Figure 5.1d and Supplementary Movie S2 (ref. 78).

Pocket $\mathrm{C}$ is formed by several amino acids including ARG95, MET97, TRP143, GLN163, and VAL177. This pocket is especially important because it is very close to the chromophore (just below the chromophore). As we show later, a mutation of GLN163 allows easier entry for the molecular oxygen from the gateway pocket to pocket $\mathrm{C}$.

\section{Oxygen Diffusion Channels Connecting Multiple Pockets}

Analysis of the trajectories of individual oxygen molecules showed two vulnerable regions of the protein through which molecular oxygen can enter and ultimately reach pocket $\mathrm{C}$, which is directly underneath the chromophore. As described above, one of these regions on the barrel close to pocket $\mathrm{C}$ is a gap on the surface between strands $\beta 7$ and $\beta 10$. The other entry region is near pocket $A$ and further away from the chromophore. Residues HIS25, PRO55, MET136, PRO134 and TYR173 act as the gateway residues in this region (near pocket A). 
a)

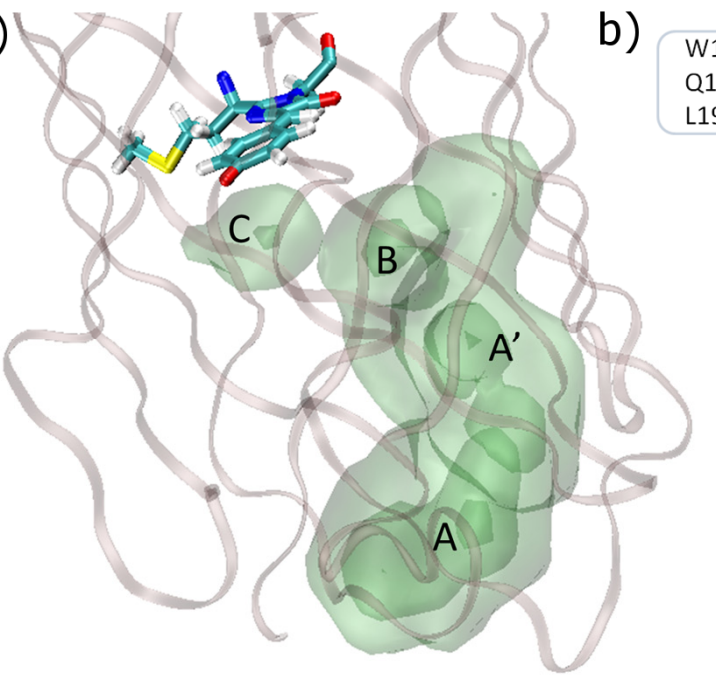

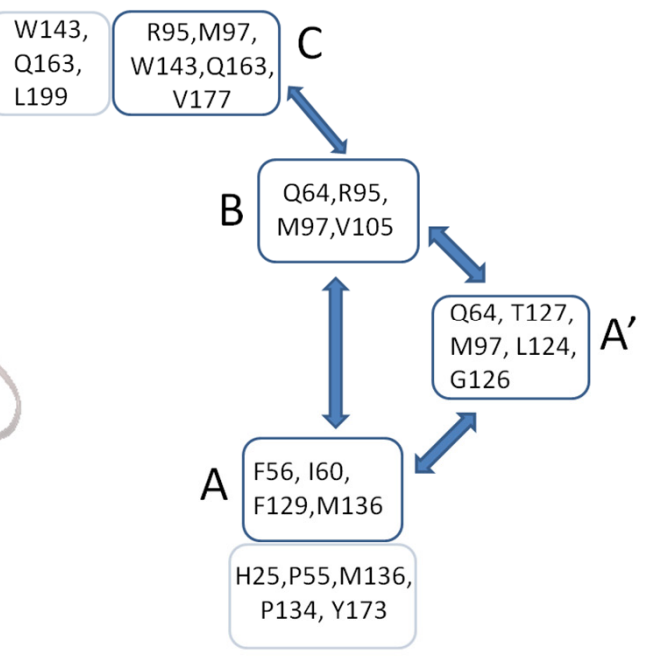

Figure 5.3 (a) Volumetric trace of the oxygen diffusion channel from one side of the protein to the other. (b) Amino acid residues involved in the oxygen diffusion channel. Common amino acid residues shared by different pockets indicate that the side chains of these residues separate the pockets. In addition to the amino acids involved in defining the different pockets, the gateway residues are also shown in light colored boxes just outside pocket $\mathrm{A}$ and pocket $\mathrm{C}$.

A complete passage of an oxygen molecule that enters from one side of the protein and leaves through the other side (solvent $\rightarrow$ pocket $\mathrm{A} \leftrightarrow \mathrm{B} \rightarrow$ pocket $\mathrm{C} \rightarrow \beta 7$ $\beta 10$ gateway pocket $\rightarrow$ solvent) was observed from 51 ns to $63 \mathrm{~ns}$ of simulation. During this time, we also observed the diffusion of oxygen between pocket $A \leftrightarrow$ pocket $A^{\prime}$. The reverse pathway was observed at $71 \mathrm{~ns}$ in which an oxygen molecule entered through the gap between $\beta 7-\beta 10$ and traveled a path from solvent $\rightarrow \beta 7-\beta 10$ gateway pocket $\rightarrow$ pocket $\mathrm{C} \rightarrow$ pocket $\mathrm{B} \rightarrow$ pocket $\mathrm{A} \rightarrow$ solvent. The oxygen molecule that entered the barrel at $71 \mathrm{~ns}$ through $\beta 7-\beta 10$ ultimately escaped from the bottom of the barrel at $73 \mathrm{~ns}$. 
This pathway is a complete channel from the $\beta 7-\beta 10$ gateway near pocket $\mathrm{C}$ into the barrel interior and then exiting near pocket A. Figure 5.3 summarizes the pathways and the residues involved in forming the channel for oxygen diffusion from one side of the protein to the other.

\subsection{FREE-ENERGIES ALONG THE PATHWAY CALCULATED FROM IMPLICIT LIGAND SAMPLING}

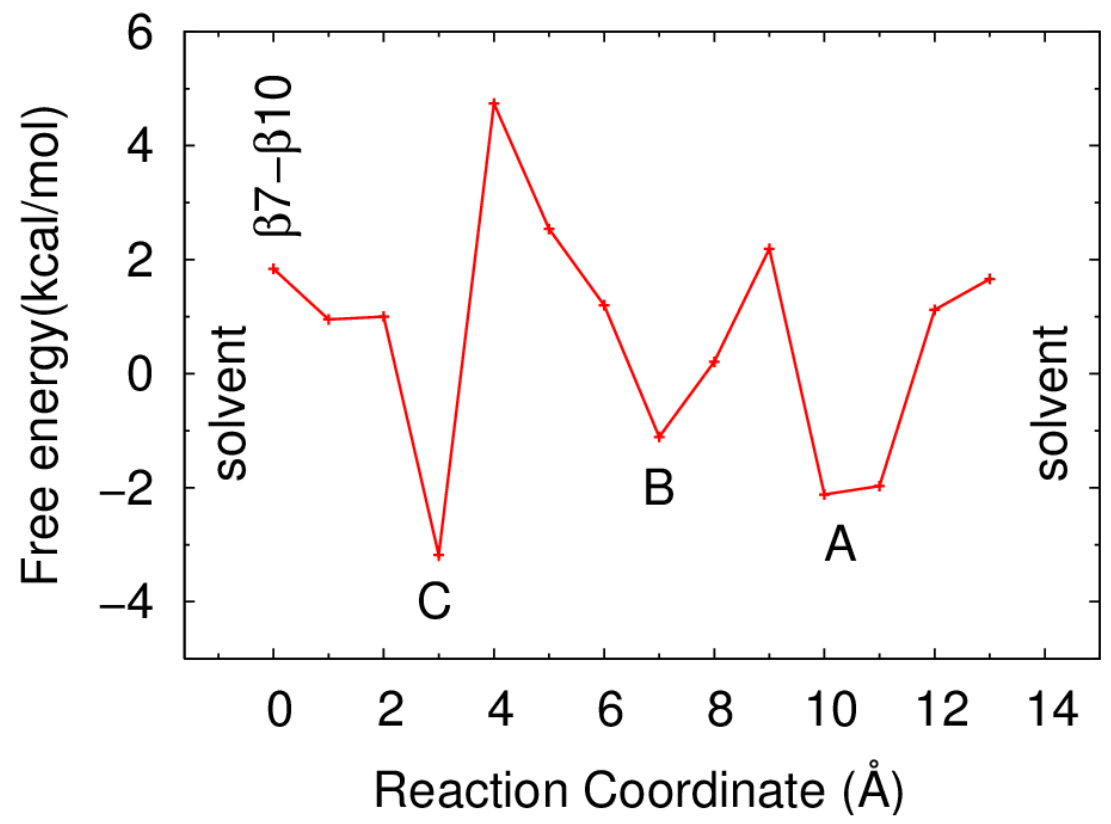

Figure 5.4 Free-energy values for the oxygen molecule at locations along the curved pathway (channel) of Figure 5.3 connecting the solvent and the protein interior. The reaction coordinate is the distance of the oxygen molecule with respect to the chromophore.

Implicit ligand sampling was performed to calculate the free-energy of an oxygen molecule at positions along the channel displayed in Figure 5.3. Figure 5.4 displays the free-energy along a reaction coordinate that represents the oxygen diffusion pathway (channel) which allows an oxygen molecule in the solvent to enter the protein and 
approach the chromophore. The free-energy curve in Figure 5.4 is based upon reaction coordinate points that are at $1 \AA$ separations along the channel. There are clear free-energy minima for pockets $\mathrm{A}, \mathrm{B}$, and $\mathrm{C}$. The barrier between pocket $\mathrm{A}$ and pocket $\mathrm{B}$ is relatively small, which indicates that it is relatively easy for the oxygen to move back and forth between these pockets. This is consistent with the short oscillation times displayed in the red curve of Figure 5.2. In contrast, the barrier between pocket $\mathrm{B}$ and $\mathrm{C}$ is much higher. The high free-energy barrier makes it more difficult for the oxygen molecule to travel between these pockets. During the 80 ns LES simulation, only a total of three B $\leftrightarrow$ C barrier crossing events: two from pocket $\mathrm{B}$ to pocket $\mathrm{C}$ and one from pocket $\mathrm{C}$ to pocket B were observed.

\subsection{M163Q MUTATION AND THE ENHANCED PHOTOSTABILITY OF mCHERRY}

During the directed evolution of mCherry from mRFP1, the mutation M163Q was experimentally determined to be solely responsible for enhanced photostability in mCherry (22). In order to understand the role of molecular oxygen in fluorescent protein photostability, we performed simulations with the reverse mutation Q163M in mCherry and investigated the oxygen diffusion pathways in the mutant mCherry-Q163M. We performed both LES as well as a number of independent simulations. A 30-ns LES simulation with 15 copies of molecular oxygen was performed. As with the mCherry simulations, the 15 copies were placed just outside the protein barrel. Within the 30 -ns simulation, a total of five molecular oxygen entries were observed into the barrel, three through the gateway pocket near the $\beta 7-\beta 10$ gap, and two from the bottom of the barrel. (In contrast, there was no molecular oxygen entry into the mCherry barrel through the 
gateway pocket near the $\beta 7-\beta 10$ gap until $71 \mathrm{~ns}$.) The first close approach of molecular oxygen to the chromophore in mCherry-Q163M occurs fairly quickly, at $11 \mathrm{~ns}$, and this oxygen enters through the gateway pocket near the $\beta 7-\beta 10$ gap. The distance of closest approach between the phenolic oxygen in the chromophore and the molecular oxygen was $2.5 \AA$. The oxygen molecule then quickly transitions further into pocket $\mathrm{C}$.

In two other oxygen entries (at $14 \mathrm{~ns}$ and $26 \mathrm{~ns}$ ) through this gateway pocket in mCherry-Q163M, the molecular oxygen continued diffusing further into the protein but through a new route, not observed in mCherry. Rather than diffusing into pocket $\mathrm{C}$, these oxygen molecules slide through a barrel-chromophore interface and ultimately reach the back of the chromophore, making contact with the MET part of the chromophore. These oxygen molecules remain in this region for the rest of the 30 -ns simulations. Molecular oxygen entries from the bottom of the protein barrel were observed at $17 \mathrm{~ns}$ and $23 \mathrm{~ns}$ following a similar route as in mCherry (i.e. pocket $\mathrm{A} \leftrightarrow$ pocket $\mathrm{B} \leftrightarrow$ pocket $\mathrm{C}$ ). Thus, the LES simulations show that the same oxygen diffusion channels in mCherry were also observed in mCherry-Q163M. However, the rate of entry as well as the number of oxygen molecules was found to be significantly higher in mCherry-Q163M as compared to mCherry, implying a correlation between reduced oxygen permeability and the improved photostability in mCherry.

In order to further investigate differences in the mechanism of oxygen diffusion, as was done with mCherry, for mCherry-Q163M 20 independent simulations were performed in which an oxygen molecule was placed just outside the protein barrel but near the chromophore (close to the gateway pocket). The initial location of the molecular oxygen is comparable to the location of the oxygen used in the mCherry simulations: the 
distance between the chromophore phenolic oxygen and the molecular oxygen was 2.69 $\AA$ in mCherry versus $2.70 \AA$ in mCherry-Q163M. In the 20 runs with mCherry-Q163M, molecular oxygen was observed to enter the gateway pocket in 14 runs. In 5 of these runs, molecular oxygen diffused further into pocket C. In contrast, simulations in mCherry showed the diffusion of molecular oxygen into pocket $\mathrm{C}$ in only one run. Also, in mCherry-Q163M, we observed molecular oxygen making back and forth transitions between the gateway pocket and pocket $\mathrm{C}$, but did not observe this in mCherry.

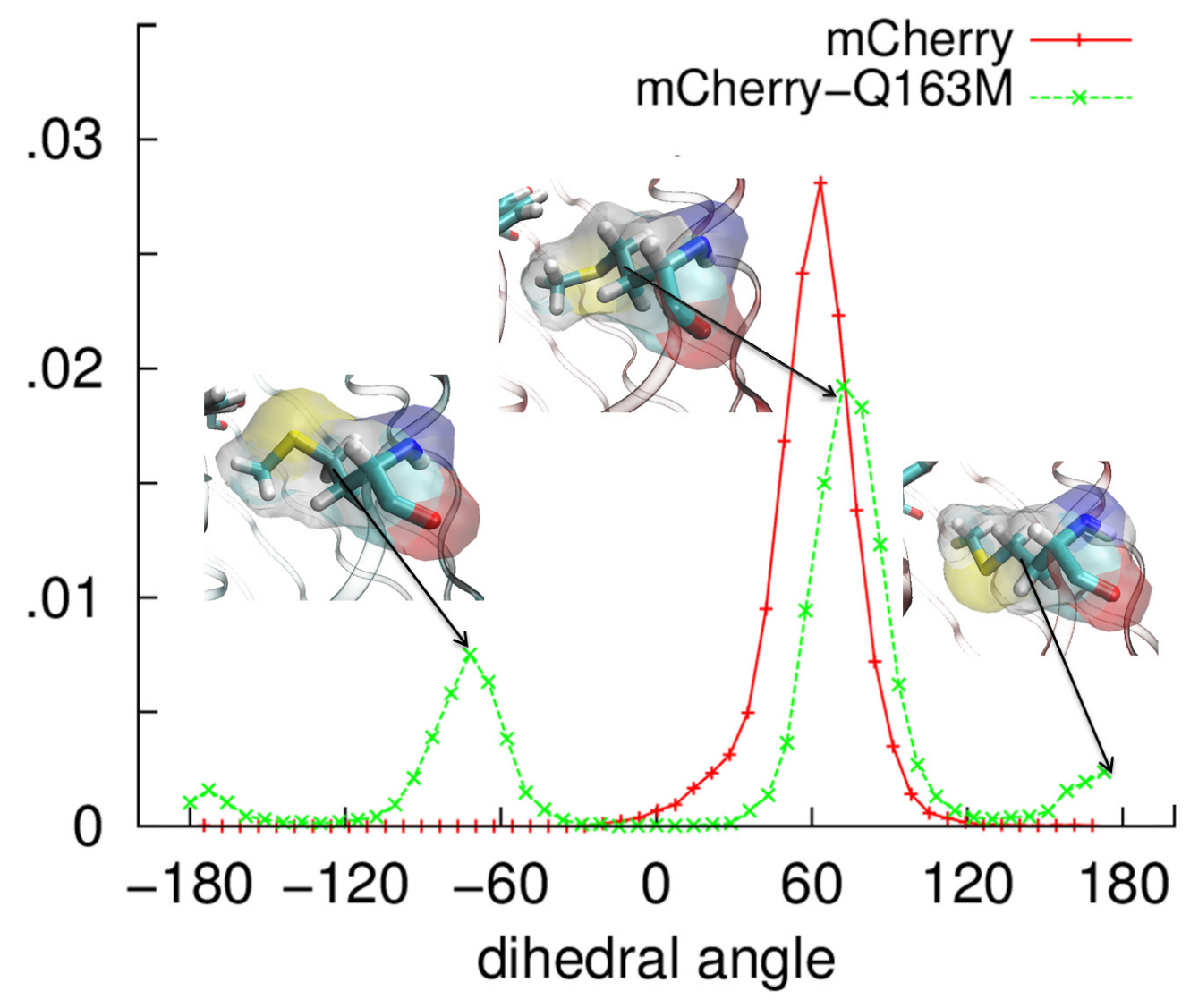

Figure 5.5 Distribution and schematic of side chain dihedral angles for the gateway residue GLN163 in mCherry versus MET163 in mCherry-Q163M. The MET163 is much more flexible and is more likely to allow diffusion of molecular oxygen into the protein compared to the more rigid GLN163. 
In order to understand the structural basis for the differences in the oxygen diffusion between gateway pocket $\leftrightarrow$ pocket $\mathrm{C}$, we inspected the dynamics of the amino acid side chain involved in the interface between these pockets. The crucial side chain at the interface is residue 163, which is GLN163 in mCherry versus MET163 in mCherryQ163M. Although the sizes of the GLN and MET side chains are quite similar, we found their dynamics to be different. In Figure 5.5 we plot the distribution of the dihedral angles (C-C-C-N and C-C-S-C) of GLN163 in mCherry and MET163 in mCherry-Q163M. The distributions of dihedral angles are obtained from a 25-ns window within their respective LES simulations. The dihedral angle distributions clearly show that the GLN163 side chain in mCherry is more rigid than the MET163 side chain in mCherry-Q163M.

The side chain of MET163 can flip between two structures with very different peak dihedral angles of $-72^{\circ}$ and $+72^{\circ}$. In addition, there is a probability for the MET163 side chain to assume a dihedral angle of $180^{\circ}$, which is not observed for GLN163. Although a combination of chromophore positions as well as the position of nearby atoms plays a role in the gateway pocket $\leftrightarrow$ pocket $\mathrm{C}$ oxygen transitions, a number of such oxygen transitions was observed when the dihedral angle was close to $180^{\circ}$. We also observed that the mutation at 163 causes the rearrangement of nearby residues, such as a shift in the dihedral angle distribution of MET97, which might alter other oxygen diffusion pathways. 


\section{CONCLUSIONS}

Access for molecular oxygen to get inside the protein barrel is required for chromophore maturation in fluorescent proteins. However, oxygen access can also cause irreversible photobleaching and significantly reduce the photostabilty of an FP. In this work, we performed molecular dynamics simulations to investigate the diffusion of molecular oxygen into the protein barrel of the monomeric RFP variant mCherry. A clear channel for oxygen diffusion into the protein was described, and the free-energy of an oxygen molecule at any point along the path has been calculated. The pathway contains several oxygen hosting pockets, which are identified by the amino acid residues that form each pocket. One end of the channel is accessed from the solvent through the floppy $\beta 7$ $\beta 10$ gap, which leads immediately to a gateway pocket that provides some access to the chromophore. Diffusion of molecular oxygen deeper into the protein, providing better access to the chromophore, is hindered by GLN163, but our calculations show that it becomes easier upon the mutation Q163M, which is consistent with experimental observations of significantly lower photostability for mCherry-Q163M as compared to that of mCherry. Another entrance from the solvent is at the bottom of the protein barrel and leads through other pockets to join the same pocket next to the chromophore. The oxygen access to the chromophore through regions close to $\beta 7$ not only leads to collisional quenching but also affects the protein's fluorescence lifetime. Such computational identifications of oxygen diffusion pathways can be helpful in guiding mutagenesis efforts to design fluorescent proteins with improved photophysical properties. We have also shown that specific point mutations can alter the oxygen pathways in the RFPs. 
Blocking or altering these pathways through the barrel can have an effect on FP maturation as well as on its photostability. For example, easy oxygen access may significantly reduce the photostability whereas it may be useful for chromophore maturation, especially at low oxygen conditions. The computational approach can provide important insights for guiding efficient mutagenesis experiments to improve the maturation speed and photostability of mFruits. 


\section{REFERENCES}

1. Tsien RY. The green fluorescent protein. Annu Rev Biochem. 1998;67:509-44. Epub 1998/10/06. doi: 10.1146/annurev.biochem.67.1.509. PubMed PMID: 9759496.

2. Zimmer M. Green fluorescent protein (GFP): applications, structure, and related photophysical behavior. Chem Rev. 2002;102(3):759-81. Epub 2002/03/14. doi: cr010142r [pii]. PubMed PMID: 11890756.

3. Chalfie M, Tu Y, Euskirchen G, Ward WW, Prasher DC. Green fluorescent protein as a marker for gene expression. Science. 1994;263(5148):802-5. Epub 1994/02/11. PubMed PMID: 8303295.

4. Shimomura O, Johnson FH, Saiga Y. Extraction, purification and properties of aequorin, a bioluminescent protein from the luminous hydromedusan, Aequorea. Journal of cellular and comparative physiology. 1962;59:223-39. PubMed PMID: 13911999.

5. Roitberg A, Elber R. Modeling side chains in peptides and proteins: Application of the locally enhanced sampling and the simulated annealing methods to find minimum energy conformations. The Journal of Chemical Physics. 1991;95(12):9277-87. doi: doi:http://dx.doi.org/10.1063/1.461157.

6. Kumagai A, Ando R, Miyatake H, Greimel P, Kobayashi T, Hirabayashi Y, et al. A bilirubin-inducible fluorescent protein from eel muscle. Cell. 2013;153(7):1602-11. doi: 10.1016/j.cell.2013.05.038. PubMed PMID: 23768684.

7. Webber NM, Litvinenko KL, Meech SR. Radiationless Relaxation in a Synthetic Analogue of the Green Fluorescent Protein Chromophore. The Journal of Physical Chemistry B. 2001;105(33):8036-9. doi: 10.1021/jp011430u.

8. Shaner NC, Patterson GH, Davidson MW. Advances in fluorescent protein technology. Journal of cell science. 2007;120(Pt 24):4247-60. doi: 10.1242/jcs.005801. PubMed PMID: 18057027.

9. Heim R, Cubitt AB, Tsien RY. Improved green fluorescence. Nature. 1995;373(6516):663-4. Epub 1995/02/23. doi: 10.1038/373663b0. PubMed PMID: 7854443.

10. Heim R, Prasher DC, Tsien RY. Wavelength mutations and posttranslational autoxidation of green fluorescent protein. Proceedings of the National Academy of Sciences of the United States of America. 1994;91(26):12501-4. Epub 1994/12/20. PubMed PMID: 7809066; PubMed Central PMCID: PMCPMC45466. 
11. Ormo M, Cubitt AB, Kallio K, Gross LA, Tsien RY, Remington SJ. Crystal structure of the Aequorea victoria green fluorescent protein. Science. 1996;273(5280):1392-5. PubMed PMID: 8703075.

12. Matz MV, Fradkov AF, Labas YA, Savitsky AP, Zaraisky AG, Markelov ML, et al. Fluorescent proteins from nonbioluminescent Anthozoa species. Nature biotechnology. 1999;17(10):969-73. Epub 1999/10/03. doi: 10.1038/13657. PubMed PMID: 10504696.

13. Lin MZ, McKeown MR, Ng HL, Aguilera TA, Shaner NC, Campbell RE, et al. Autofluorescent proteins with excitation in the optical window for intravital imaging in mammals. Chem Biol. 2009;16(11):1169-79. Epub 2009/11/28. doi: S10745521(09)00360-3 [pii] 10.1016/j.chembiol.2009.10.009. PubMed PMID: 19942140; PubMed Central PMCID: PMCPMC2814181.

14. Goulding A, Shrestha S, Dria K, Hunt E, Deo SK. Red fluorescent protein variants with incorporated non-natural amino acid analogues. Protein Eng Des Sel. 2008;21(2):101-6. Epub 2008/01/22. doi: gzm075 [pii] 10.1093/protein/gzm075. PubMed PMID: 18203801.

15. Yarbrough D, Wachter RM, Kallio K, Matz MV, Remington SJ. Refined crystal structure of DsRed, a red fluorescent protein from coral, at 2.0-A resolution. Proceedings of the National Academy of Sciences of the United States of America. 2001;98(2):462-7. Epub 2001/02/24. doi: 10.1073/pnas.98.2.462 98/2/462 [pii]. PubMed PMID: 11209050; PubMed Central PMCID: PMCPMC14609.

16. Campbell RE, Tour O, Palmer AE, Steinbach PA, Baird GS, Zacharias DA, et al. A monomeric red fluorescent protein. Proceedings of the National Academy of Sciences of the United States of America. 2002;99(12):7877-82. Epub 2002/06/13. doi: 10.1073/pnas.082243699 99/12/7877 [pii]. PubMed PMID: 12060735; PubMed Central PMCID: PMCPMC122988.

17. Baird GS, Zacharias DA, Tsien RY. Biochemistry, mutagenesis, and oligomerization of DsRed, a red fluorescent protein from coral. Proceedings of the National Academy of Sciences of the United States of America. 2000;97(22):11984-9. Epub 2000/10/26. doi: 10.1073/pnas.97.22.11984 97/22/11984 [pii]. PubMed PMID: 11050229; PubMed Central PMCID: PMCPMC17281.

18. Merzlyak EM, Goedhart J, Shcherbo D, Bulina ME, Shcheglov AS, Fradkov AF, et al. Bright monomeric red fluorescent protein with an extended fluorescence lifetime. Nature methods. 2007;4(7):555-7. Epub 2007/06/19. doi: nmeth1062 [pii] 10.1038/nmeth1062. PubMed PMID: 17572680.

19. Shaner NC, Campbell RE, Steinbach PA, Giepmans BN, Palmer AE, Tsien RY. Improved monomeric red, orange and yellow fluorescent proteins derived from 
Discosoma sp. red fluorescent protein. Nature biotechnology. 2004;22(12):1567-72. Epub 2004/11/24. doi: nbt1037 [pii] 10.1038/nbt1037. PubMed PMID: 15558047.

20. Shaner NC, Steinbach PA, Tsien RY. A guide to choosing fluorescent proteins. Nature methods. 2005;2(12):905-9. Epub 2005/11/22. doi: nmeth819 [pii] 10.1038/nmeth819. PubMed PMID: 16299475.

21. Subach FV, Verkhusha VV. Chromophore transformations in red fluorescent proteins. Chem Rev. 2012;112(7):4308-27. Epub 2012/05/09. doi: 10.1021/cr2001965. PubMed PMID: 22559232; PubMed Central PMCID: PMCPMC3394910.

22. Shaner NC, Lin MZ, McKeown MR, Steinbach PA, Hazelwood KL, Davidson $\mathrm{MW}$, et al. Improving the photostability of bright monomeric orange and red fluorescent proteins. Nature methods. 2008;5(6):545-51. Epub 2008/05/06. doi: nmeth.1209 [pii] 10.1038/nmeth.1209. PubMed PMID: 18454154.

23. Amara P, Andreoletti P, Jouve HM, Field MJ. Ligand diffusion in the catalase from Proteus mirabilis: a molecular dynamics study. Protein Sci. 2001;10(10):1927-35. Epub 2001/09/22. doi: 10.1110/ps.14201. PubMed PMID: 11567083; PubMed Central PMCID: PMCPMC2374231.

24. Carlson ML, Regan RM, Gibson QH. Distal cavity fluctuations in myoglobin: protein motion and ligand diffusion. Biochemistry. 1996;35(4):1125-36. Epub 1996/01/30. doi: 10.1021/bi951767k bi951767k [pii]. PubMed PMID: 8573567.

25. Bossa C, Anselmi M, Roccatano D, Amadei A, Vallone B, Brunori M, et al. Extended molecular dynamics simulation of the carbon monoxide migration in sperm whale myoglobin. Biophys J. 2004;86(6):3855-62. Epub 2004/06/11. doi: 10.1529/biophysj.103.037432 S0006-3495(04)74426-4 [pii]. PubMed PMID: 15189882; PubMed Central PMCID: PMCPMC1304287.

26. Lamb DC, Arcovito A, Nienhaus K, Minkow O, Draghi F, Brunori M, et al. Structural dynamics of myoglobin: an infrared kinetic study of ligand migration in mutants YQR and YQRF. Biophys Chem. 2004;109(1):41-58. Epub 2004/04/03. doi: 10.1016/j.bpc.2003.10.002 S0301462203002886 [pii]. PubMed PMID: 15059658.

27. Gibson QH, Regan R, Elber R, Olson JS, Carver TE. Distal pocket residues affect picosecond ligand recombination in myoglobin. An experimental and molecular dynamics study of position 29 mutants. The Journal of biological chemistry. 1992;267(31):22022-34. Epub 1992/11/05. PubMed PMID: 1429552.

28. Brunori M, Gibson QH. Cavities and packing defects in the structural dynamics of myoglobin. EMBO Rep. 2001;2(8):674-9. Epub 2001/08/09. doi: 10.1093/emboreports/kve159 2/8/674 [pii]. PubMed PMID: 11493595; PubMed Central PMCID: PMCPMC1083996. 
29. Cohen J, Kim K, King P, Seibert M, Schulten K. Finding gas diffusion pathways in proteins: application to $\mathrm{O} 2$ and $\mathrm{H} 2$ transport in $\mathrm{CpI}$ [FeFe]-hydrogenase and the role of packing defects. Structure. 2005;13(9):1321-9. Epub 2005/09/13. doi: S09692126(05)00239-X [pii] 10.1016/j.str.2005.05.013. PubMed PMID: 16154089.

30. Feher VA, Baldwin E, Dahlquist FW. Access of ligands to cavities within the core of a protein is rapid. Nature structural biology. 1996;3:516-21.

31. Lakowicz JR, Weber G. Quenching of protein fluorescence by oxygen. Detection of structural fluctuations in proteins on the nanosecond time scale. Biochemistry. 1973;12(21):4171-9. Epub 1973/10/09. PubMed PMID: 4200894.

32. Nadler W, Stein DL. Biological transport processes and space dimension. Proceedings of the National Academy of Sciences of the United States of America. 1991;88(15):6750-4. Epub 1991/08/01. PubMed PMID: 1713690; PubMed Central PMCID: PMCPMC52166.

33. Calhoun DB, Vanderkooi JM, Woodrow GV, 3rd, Englander SW. Penetration of dioxygen into proteins studied by quenching of phosphorescence and fluorescence. Biochemistry. 1983;22(7):1526-32. Epub 1983/03/29. PubMed PMID: 6342662.

34. Branchini BR, Nemser AR, Zimmer M. A computational analysis of the unique protein-induced tight turn that results in posttranslational chromophore formation in green fluorescent protein. J Am Chem Soc. 1998;120(1):1-6. doi: 10.1021/ja973019j.

35. Shu X, Shaner NC, Yarbrough CA, Tsien RY, Remington SJ. Novel chromophores and buried charges control color in mFruits. Biochemistry. 2006;45(32):9639-47. Epub 2006/08/09. doi: 10.1021/bi0607731. PubMed PMID: 16893165.

36. Karplus M, McCammon JA. Molecular dynamics simulations of biomolecules. Nature structural biology. 2002;9(9):646-52. doi: 10.1038/nsb0902-646. PubMed PMID: 12198485.

37. Brooks BR, Brooks CL, 3rd, Mackerell AD, Jr., Nilsson L, Petrella RJ, Roux B, et al. CHARMM: the biomolecular simulation program. Journal of computational chemistry. 2009;30(10):1545-614. doi: 10.1002/jcc.21287. PubMed PMID: 19444816; PubMed Central PMCID: PMC2810661.

38. Field MJ. A practical introduction to the simulation of molecular systems. Cambridge: Cambridge University Press; 1999.

39. Best RB, Zhu X, Shim J, Lopes PE, Mittal J, Feig M, et al. Optimization of the additive CHARMM all-atom protein force field targeting improved sampling of the 
backbone phi, psi and side-chain chi(1) and chi(2) dihedral angles. Journal of chemical theory and computation. 2012;8(9):3257-73. doi: 10.1021/ct300400x. PubMed PMID: 23341755; PubMed Central PMCID: PMC3549273.

40. Eswar N, Webb B, Marti-Renom MA, Madhusudhan MS, Eramian D, Shen MY, et al. Comparative protein structure modeling using Modeller. Current protocols in bioinformatics / editoral board, Andreas D Baxevanis [et al]. 2006; Chapter 5:Unit 56. doi: 10.1002/0471250953.bi0506s15. PubMed PMID: 18428767.

41. Feig M, Karanicolas J, Brooks CL, 3rd. MMTSB Tool Set: enhanced sampling and multiscale modeling methods for applications in structural biology. Journal of molecular graphics \& modelling. 2004;22(5):377-95. doi: 10.1016/j.jmgm.2003.12.005. PubMed PMID: 15099834.

42. Ulrich E, Lalith P, Max LB, Tom D, Hsing L, Lee GP. A smooth particle mesh Ewald method. The Journal of Chemical Physics. 1995;103(19):8577-93.

43. Reuter N, Lin H, Thiel W. Green Fluorescent Proteins: Empirical Force Field for the Neutral and Deprotonated Forms of the Chromophore. Molecular Dynamics Simulations of the Wild Type and S65T Mutant. The Journal of Physical Chemistry B. 2002;106(24):6310-21. doi: 10.1021/jp014476w.

44. Cohen J, Olsen KW, Schulten K. Finding gas migration pathways in proteins using implicit ligand sampling. Methods in enzymology. 2008;437:439-57. doi: 10.1016/S0076-6879(07)37022-5. PubMed PMID: 18433641.

45. Cohen J, Arkhipov A, Braun R, Schulten K. Imaging the migration pathways for $\mathrm{O} 2$, CO, NO, and Xe inside myoglobin. Biophys J. 2006;91(5):1844-57. Epub 2006/06/06. doi: S0006-3495(06)71896-3 [pii] 10.1529/biophysj.106.085746. PubMed PMID: 16751246; PubMed Central PMCID: PMCPMC1544290.

46. Humphrey W, Dalke A, Schulten K. VMD: visual molecular dynamics. Journal of molecular graphics. 1996;14(1):33-8, 27-8. PubMed PMID: 8744570.

47. Phillips JC, Braun R, Wang W, Gumbart J, Tajkhorshid E, Villa E, et al. Scalable molecular dynamics with NAMD. Journal of computational chemistry. 2005;26(16):1781-802. Epub 2005/10/14. doi: 10.1002/jcc.20289. PubMed PMID: 16222654; PubMed Central PMCID: PMCPMC2486339.

48. Griesbeck O, Baird GS, Campbell RE, Zacharias DA, Tsien RY. Reducing the environmental sensitivity of yellow fluorescent protein. Mechanism and applications. The Journal of biological chemistry. 2001;276(31):29188-94. doi: 10.1074/jbc.M102815200. PubMed PMID: 11387331. 
49. Miyawaki A, Nagai T, Mizuno H. Engineering fluorescent proteins. Advances in biochemical engineering/biotechnology. 2005;95:1-15. PubMed PMID: 16080263.

50. Wachter RM, Yarbrough D, Kallio K, Remington SJ. Crystallographic and energetic analysis of binding of selected anions to the yellow variants of green fluorescent protein. Journal of molecular biology. 2000;301(1):157-71. doi: 10.1006/jmbi.2000.3905. PubMed PMID: 10926499.

51. Rekas A, Alattia JR, Nagai T, Miyawaki A, Ikura M. Crystal structure of venus, a yellow fluorescent protein with improved maturation and reduced environmental sensitivity. The Journal of biological chemistry. 2002;277(52):50573-8. doi: 10.1074/jbc.M209524200. PubMed PMID: 12370172.

52. Chapagain PP, Regmi CK, Castillo W. Fluorescent protein barrel fluctuations and oxygen diffusion pathways in mCherry. J Chem Phys. 2011;135(23):235101. Epub 2011/12/24. doi: 10.1063/1.3660197. PubMed PMID: 22191901; PubMed Central PMCID: PMCPMC3248888.

53. Yang F, Moss LG, Phillips GN, Jr. The molecular structure of green fluorescent protein. Nature biotechnology. 1996;14(10):1246-51. doi: 10.1038/nbt1096-1246. PubMed PMID: 9631087.

54. Stepanenko OV, Stepanenko OV, Shcherbakova DM, Kuznetsova IM, Turoverov KK, Verkhusha VV. Modern fluorescent proteins: from chromophore formation to novel intracellular applications. BioTechniques. 2011;51(5):313-4, 6, 8 passim. doi: 10.2144/000113765. PubMed PMID: 22054544.

55. Miyawaki A, Nagai T, Mizuno H. Mechanisms of protein fluorophore formation and engineering. Current opinion in chemical biology. 2003;7(5):557-62. PubMed PMID: 14580558.

56. Subach FV, Piatkevich KD, Verkhusha VV. Directed molecular evolution to design advanced red fluorescent proteins. Nature methods. 2011;8(12):1019-26. doi: 10.1038/nmeth.1776. PubMed PMID: 22127219.

57. Grigorenko BL, Nemukhin AV, Polyakov IV, Morozov DI, Krylov AI. Firstprinciples characterization of the energy landscape and optical spectra of green fluorescent protein along the A-->I-->B proton transfer route. Journal of the American Chemical Society. 2013;135(31):11541-9. doi: 10.1021/ja402472y. PubMed PMID: 23837665.

58. Liu RS. Photoisomerization by hula-twist: a fundamental supramolecular photochemical reaction. Accounts of chemical research. 2001;34(7):555-62. PubMed PMID: 11456473. 
59. Megley CM, Dickson LA, Maddalo SL, Chandler GJ, Zimmer M. Photophysics and dihedral freedom of the chromophore in yellow, blue, and green fluorescent protein. The journal of physical chemistry B. 2009;113(1):302-8. doi: 10.1021/jp806285s. PubMed PMID: 19067572; PubMed Central PMCID: PMC2671006.

60. Mizuno H, Mal TK, Walchli M, Kikuchi A, Fukano T, Ando R, et al. Lightdependent regulation of structural flexibility in a photochromic fluorescent protein. Proceedings of the National Academy of Sciences of the United States of America. 2008;105(27):9227-32. doi: 10.1073/pnas.0709599105. PubMed PMID: 18574155; PubMed Central PMCID: PMC2453726.

61. Justin RG, Rosemary B, Klaus S. Reconstructing potentials of mean force through time series analysis of steered molecular dynamics simulations. J Comput Phys. 1999;151(1):190-211. doi: http://dx.doi.org/10.1006/jcph.1999.6218.

62. Roux B. The calculation of the potential of mean force using computer simulations. Comp Phys Commun. 1995;91(1-3):275-82.

63. Wang Y, Cohen J, Boron WF, Schulten K, Tajkhorshid E. Exploring gas permeability of cellular membranes and membrane channels with molecular dynamics. J Struct Biol. 2007;157(3):534-44. Epub 2007/02/20. doi: S1047-8477(06)00378-9 [pii] 10.1016/j.jsb.2006.11.008. PubMed PMID: 17306562.

64. Johnson BJ, Cohen J, Welford RW, Pearson AR, Schulten K, Klinman JP, et al. Exploring molecular oxygen pathways in Hansenula polymorpha copper-containing amine oxidase. The Journal of biological chemistry. 2007;282(24):17767-76. Epub 2007/04/06. doi: M701308200 [pii] 10.1074/jbc.M701308200. PubMed PMID: 17409383.

65. Saam J, Ivanov I, Walther M, Holzhutter HG, Kuhn H. Molecular dioxygen enters the active site of 12/15-lipoxygenase via dynamic oxygen access channels. Proceedings of the National Academy of Sciences of the United States of America. 2007;104(33):13319-24. Epub 2007/08/07. doi: 0702401104 [pii] 10.1073/pnas.0702401104. PubMed PMID: 17675410; PubMed Central PMCID: PMCPMC1948941.

66. Saam J, Rosini E, Molla G, Schulten K, Pollegioni L, Ghisla S. O2 reactivity of flavoproteins: dynamic access of dioxygen to the active site and role of a $\mathrm{H}+$ relay system in D-amino acid oxidase. J Biol Chem. 2010;285(32):24439-46. Epub 2010/05/26. doi: M110.131193 [pii] 10.1074/jbc.M110.131193. PubMed PMID: 20498362; PubMed Central PMCID: PMCPMC2915680.

67. Baron R, Riley C, Chenprakhon P, Thotsaporn K, Winter RT, Alfieri A, et al. Multiple pathways guide oxygen diffusion into flavoenzyme active sites. Proceedings of the National Academy of Sciences of the United States of America. 2009;106(26):10603- 
8. Epub 2009/06/23. doi: 0903809106 [pii] 10.1073/pnas.0903809106. PubMed PMID: 19541622; PubMed Central PMCID: PMCPMC2698890.

68. Fan Y, Lund L, Shao Q, Gao YQ, Raushel FM. A combined theoretical and experimental study of the ammonia tunnel in carbamoyl phosphate synthetase. J Am Chem Soc. 2009;131(29):10211-9. Epub 2009/07/03. doi: 10.1021/ja902557r. PubMed PMID: 19569682; PubMed Central PMCID: PMCPMC2748306.

69. Lund L, Fan Y, Shao Q, Gao YQ, Raushel FM. Carbamate transport in carbamoyl phosphate synthetase: a theoretical and experimental investigation. J Am Chem Soc.132(11):3870-8. Epub 2010/03/02. doi: 10.1021/ja910441v. PubMed PMID: 20187643; PubMed Central PMCID: PMCPMC2847351.

70. Wang XS, Roitberg AE, Richards NG. Computational studies of ammonia channel function in glutamine 5'-phosphoribosylpyrophosphate amidotransferase. Biochemistry. 2009;48(51):12272-82. Epub 2009/11/20. doi: 10.1021/bi901521d. PubMed PMID: 19921932.

71. Daigle R, Guertin M, Lague P. Structural characterization of the tunnels of Mycobacterium tuberculosis truncated hemoglobin $\mathrm{N}$ from molecular dynamics simulations. Proteins. 2009;75(3):735-47. Epub 2008/11/13. doi: 10.1002/prot.22283. PubMed PMID: 19003999.

72. Craggs TD. Green fluorescent protein: structure, folding and chromophore maturation. Chem Soc Rev. 2009;38(10):2865-75. Epub 2009/09/23. doi: 10.1039/b903641p. PubMed PMID: 19771333.

73. Evdokimov AG, Pokross ME, Egorov NS, Zaraisky AG, Yampolsky IV, Merzlyak EM, et al. Structural basis for the fast maturation of Arthropoda green fluorescent protein. EMBO Rep. 2006;7(10):1006-12. Epub 2006/08/29. doi: 7400787 [pii] 10.1038/sj.embor.7400787. PubMed PMID: 16936637; PubMed Central PMCID: PMCPMC1618374.

74. Wang PH, Blumberger J. Mechanistic insight into the blocking of $\mathrm{CO}$ diffusion in [NiFe]-hydrogenase mutants through multiscale simulation. Proceedings of the National Academy of Sciences of the United States of America. 2012;109(17):6399-404. Epub 2012/04/12. doi: 10.1073/pnas.1121176109 1121176109 [pii]. PubMed PMID: 22493222; PubMed Central PMCID: PMCPMC3340082.

75. Goedhart J, von Stetten D, Noirclerc-Savoye M, Lelimousin M, Joosen L, Hink $\mathrm{MA}$, et al. Structure-guided evolution of cyan fluorescent proteins towards a quantum yield of 93\%. Nat Commun. 2012;3:751. Epub 2012/03/22. doi: 10.1038/ncomms1738 ncomms1738 [pii]. PubMed PMID: 22434194; PubMed Central PMCID: PMCPMC3316892. 
76. Roy A, Carpentier P, Bourgeois D, Field M. Diffusion pathways of oxygen species in the phototoxic fluorescent protein KillerRed. Photochem Photobiol Sci. 2010;9(10):1342-50. Epub 2010/09/08. doi: 10.1039/c0pp00141d. PubMed PMID: 20820672.

77. Bevis BJ, Glick BS. Rapidly maturing variants of the Discosoma red fluorescent protein (DsRed). Nature biotechnology. 2002;20(1):83-7. Epub 2001/12/26. doi: 10.1038/nbt0102-83 nbt0102-83 [pii]. PubMed PMID: 11753367.

78. Regmi CK, Bhandari YR, Gerstman BS, Chapagain PP. Exploring the diffusion of molecular oxygen in the red fluorescent protein mCherry using explicit oxygen molecular dynamics simulations. The journal of physical chemistry B. 2013;117(8):224753. doi: 10.1021/jp308366y. PubMed PMID: 23363049; PubMed Central PMCID: PMC3587716. 


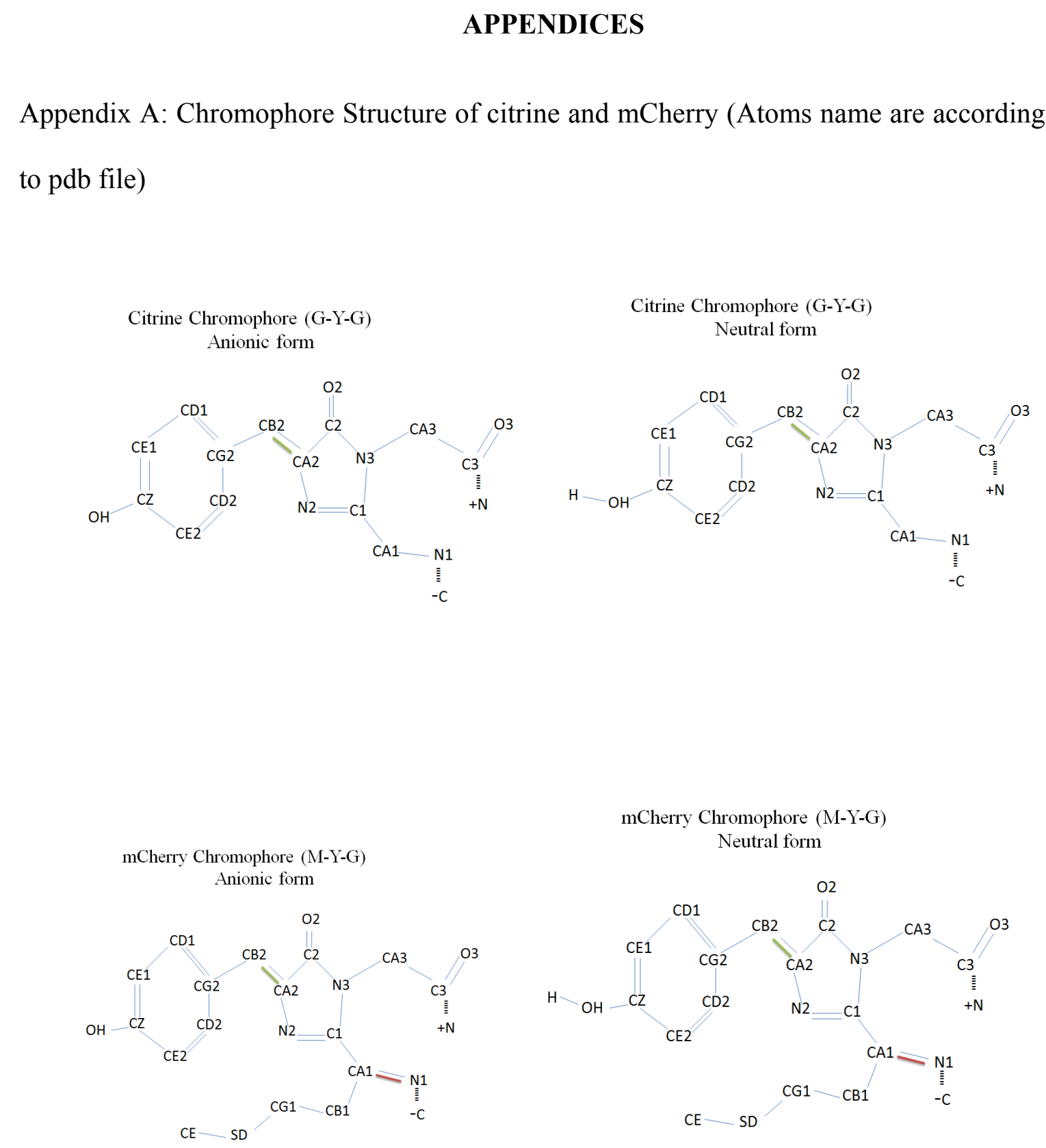


Appendix B: Residue topology and parameter files for the chromophore of mCherry

Table 5.1 Residue topology file for the chromophore of mCherry (anionic)

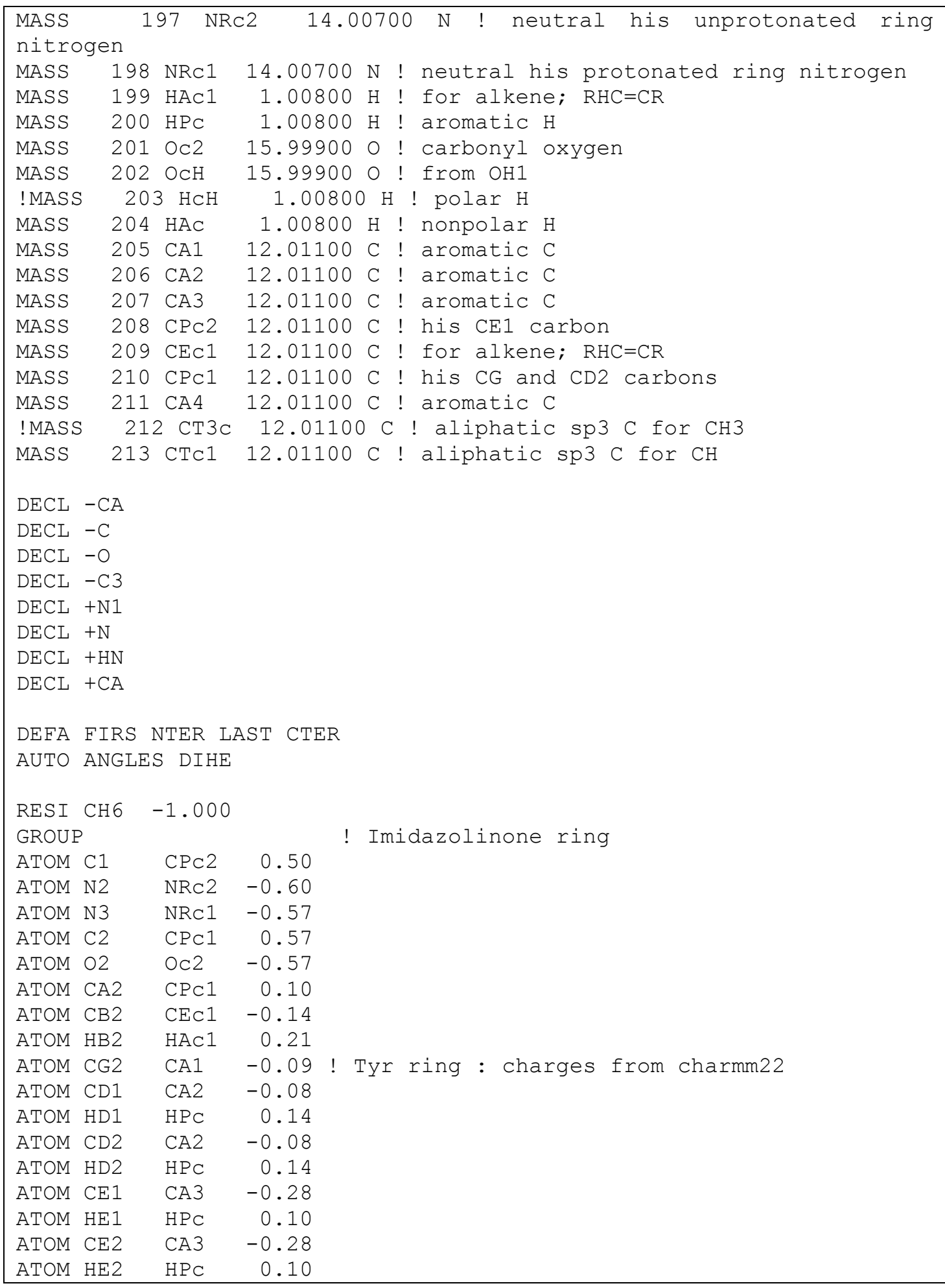




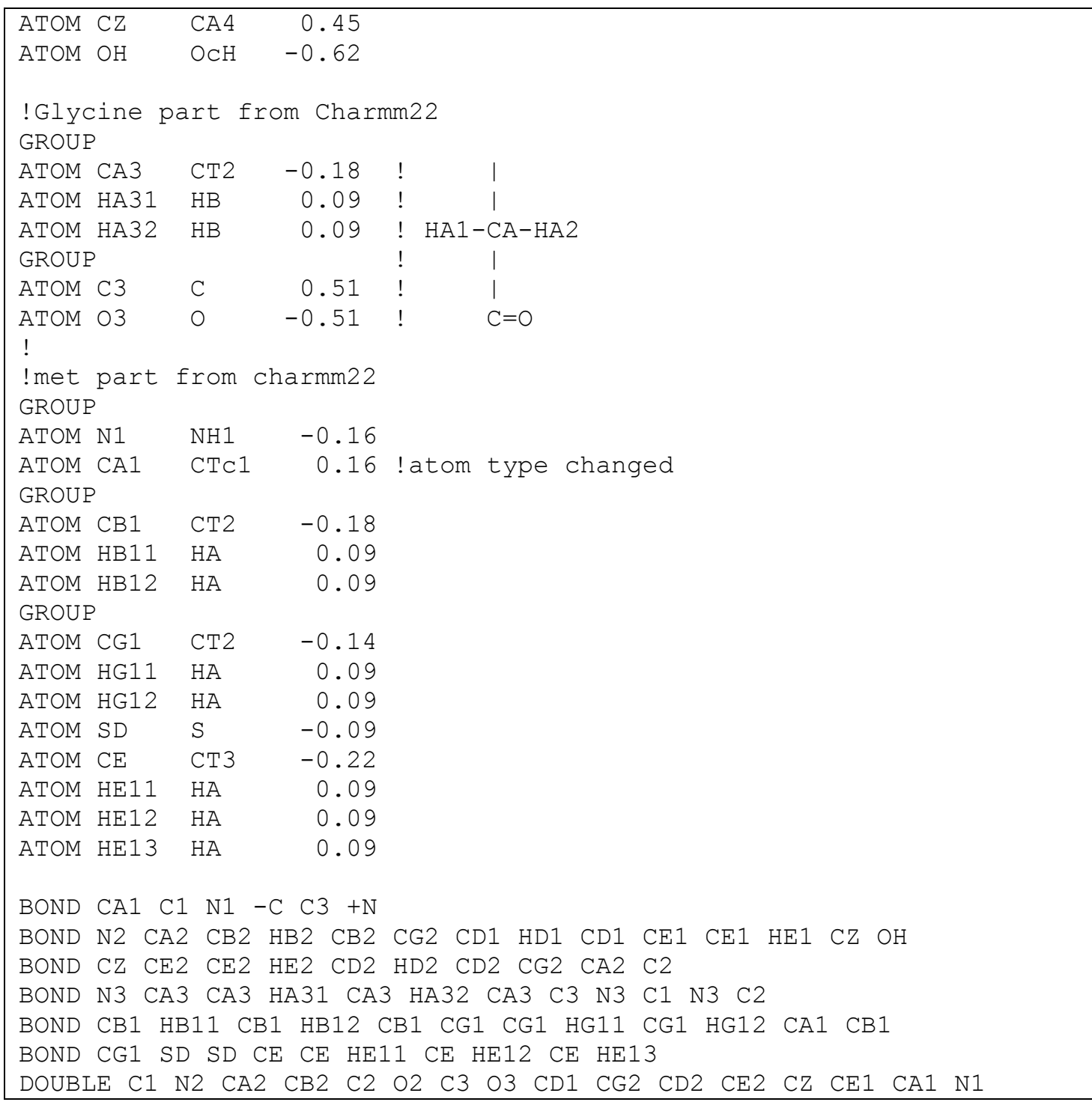

\section{Table 5.2 Parameter file for the chromophore of mCherry (anionic)}

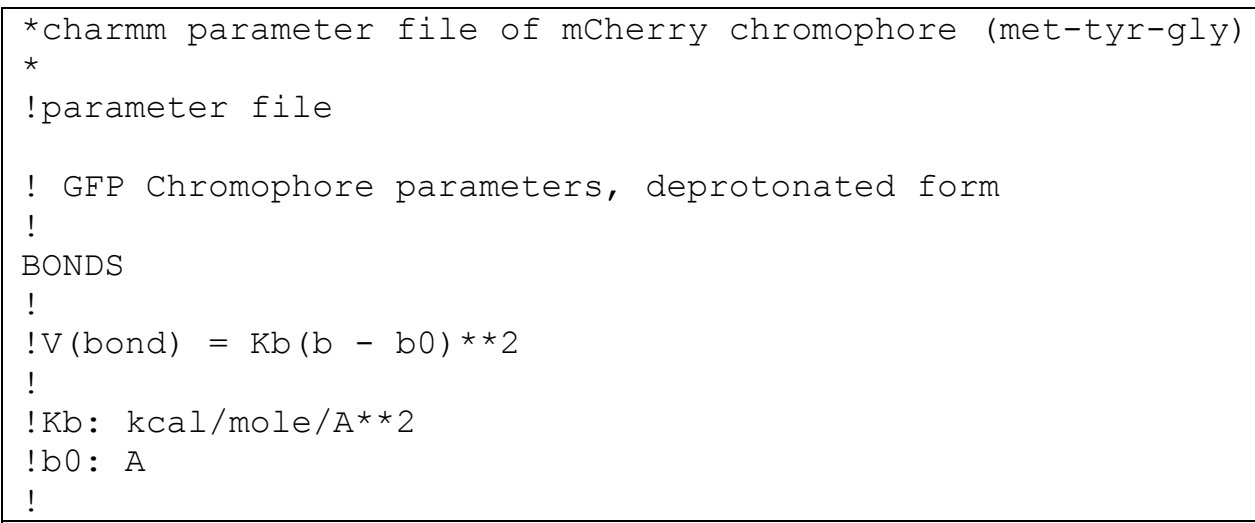




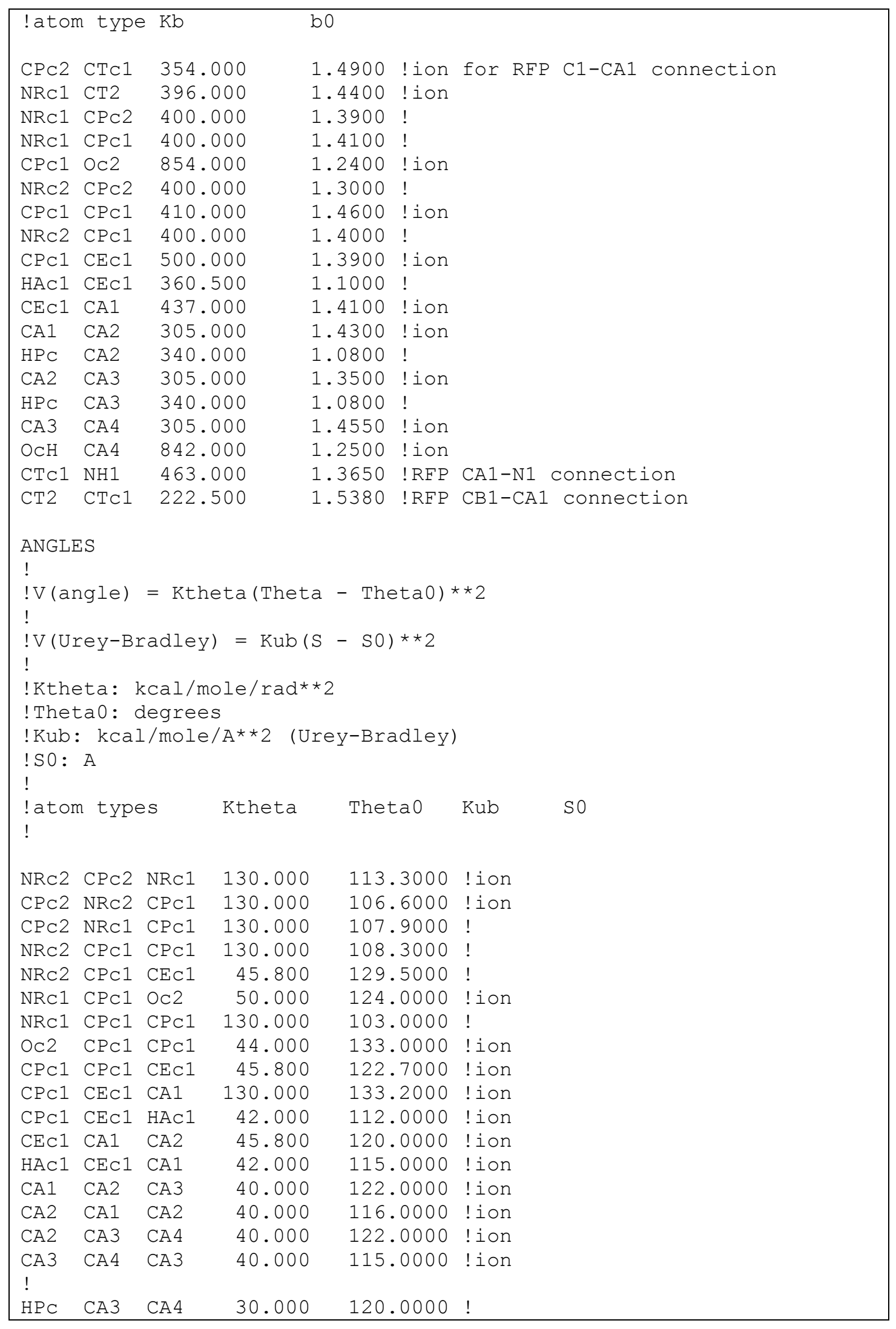




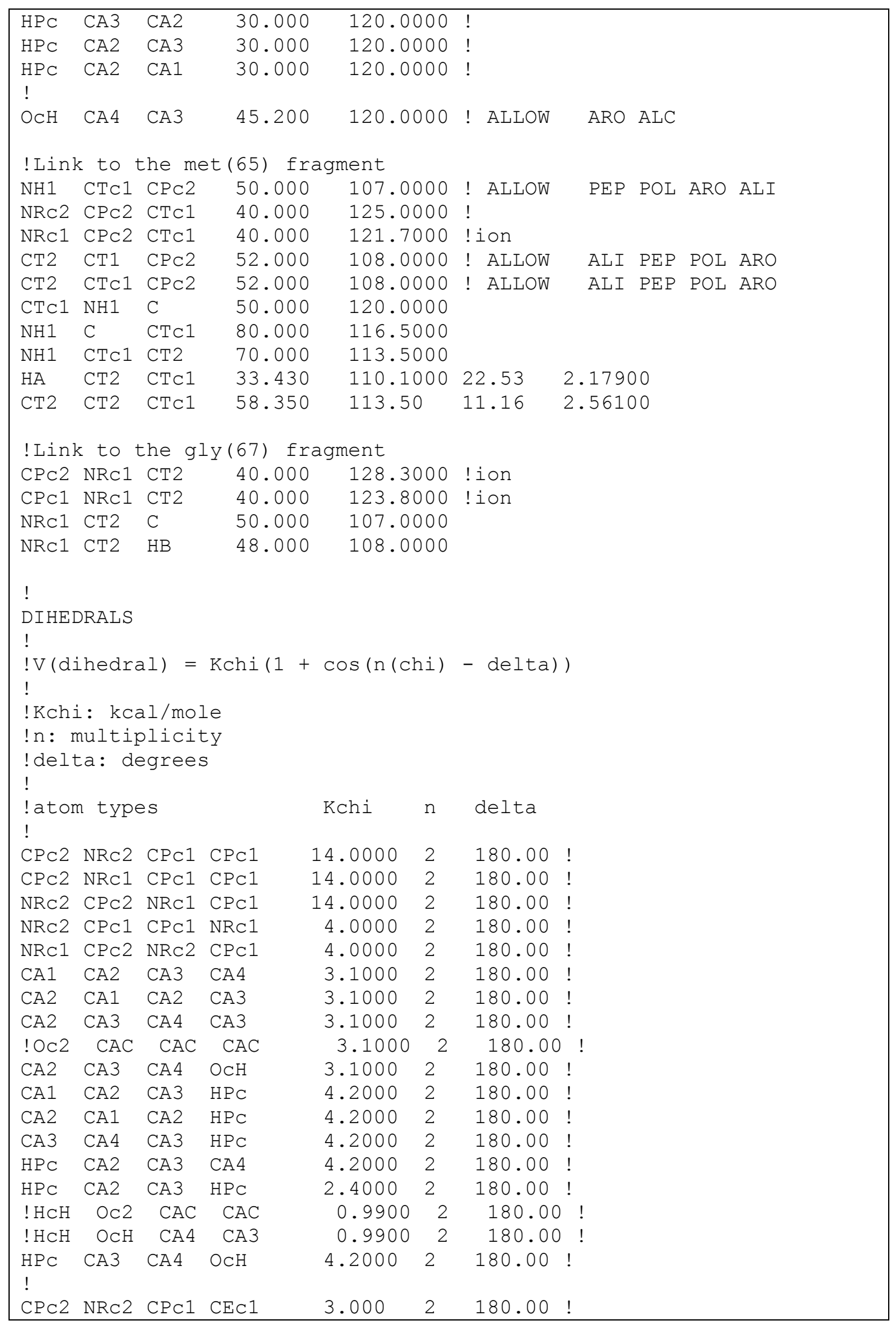




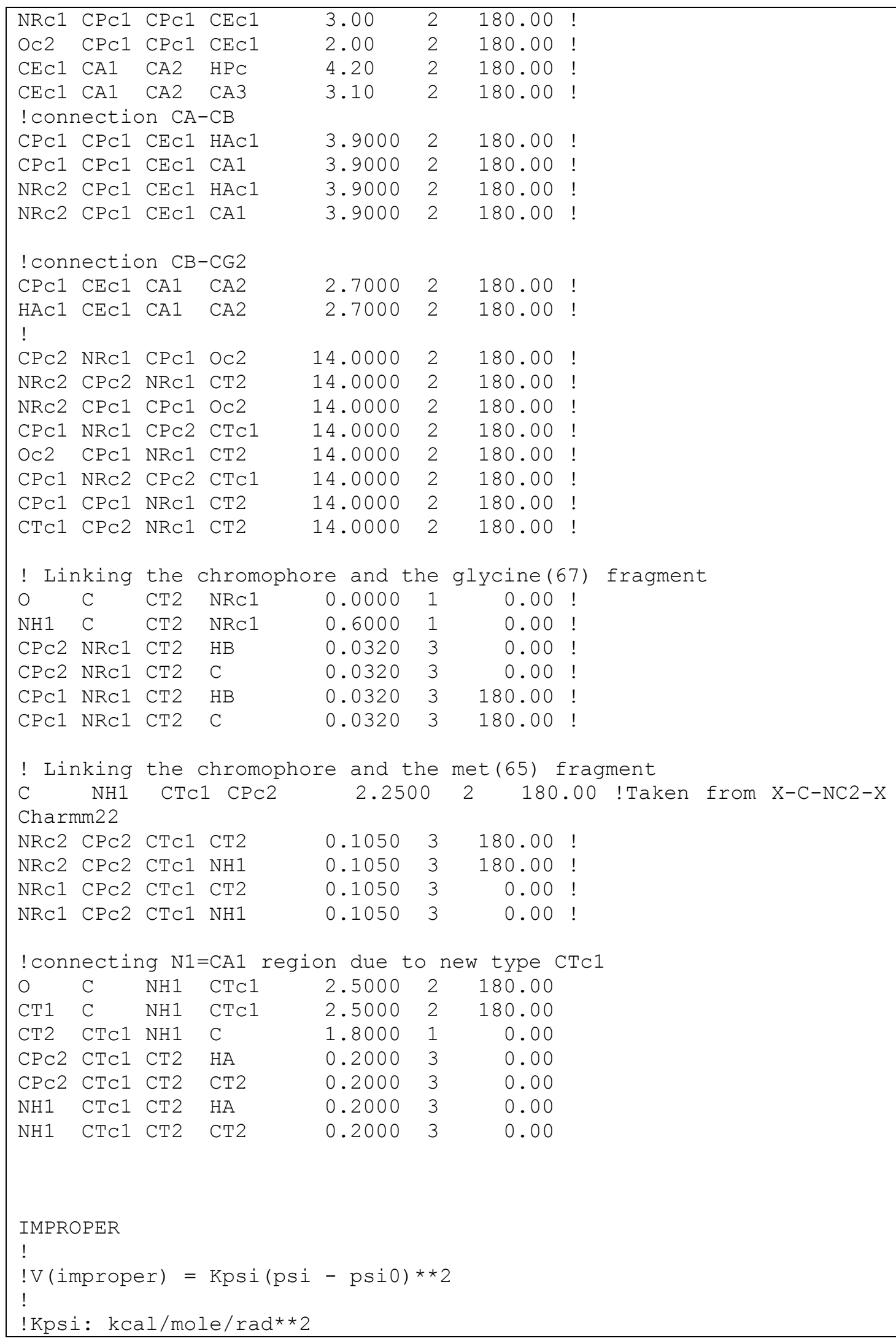




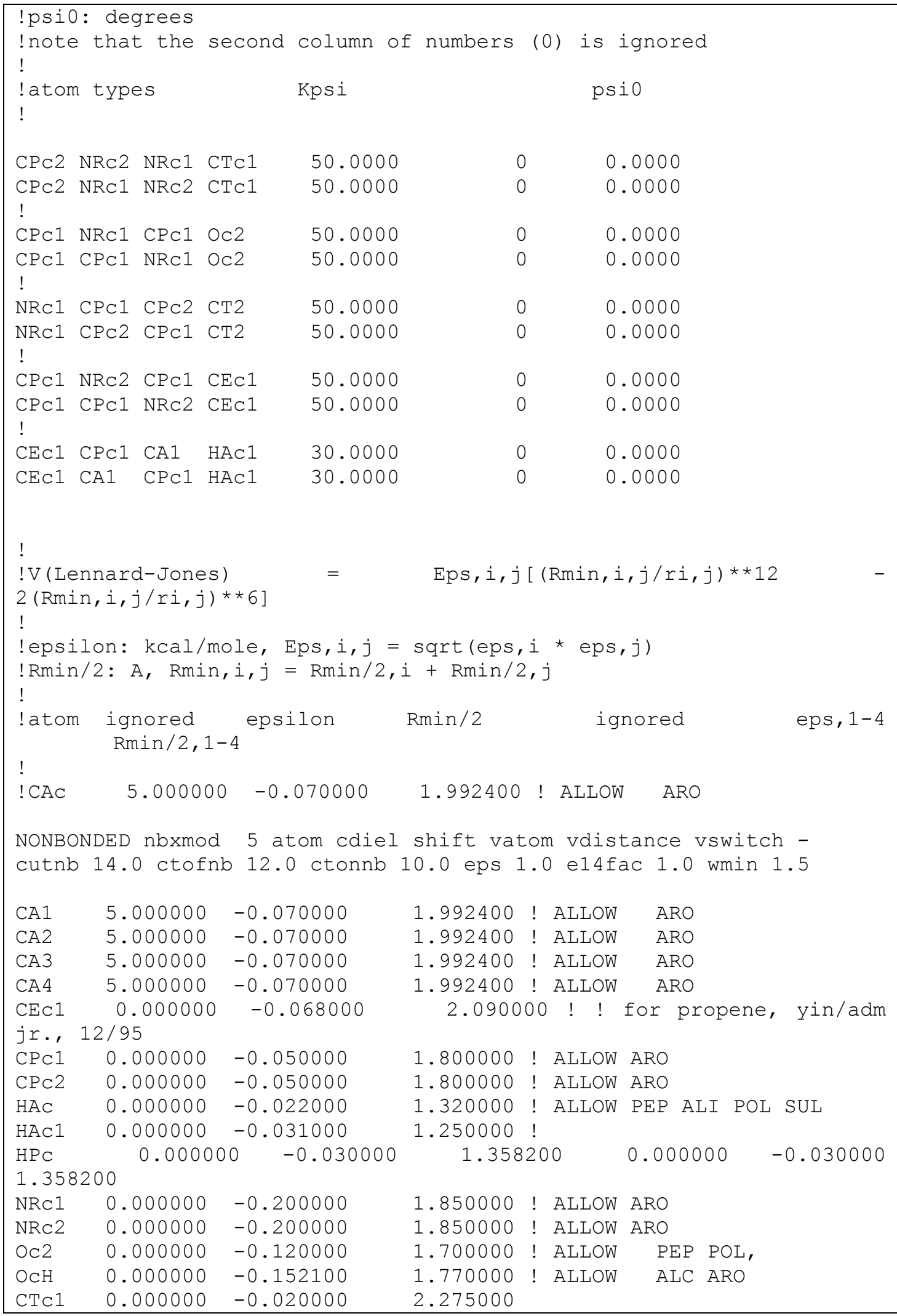


Appendix C: Residue topology and parameter files for the chromophore of citrine

Table 5.3 Residue topology file for the chromophore of citrine (anionic)

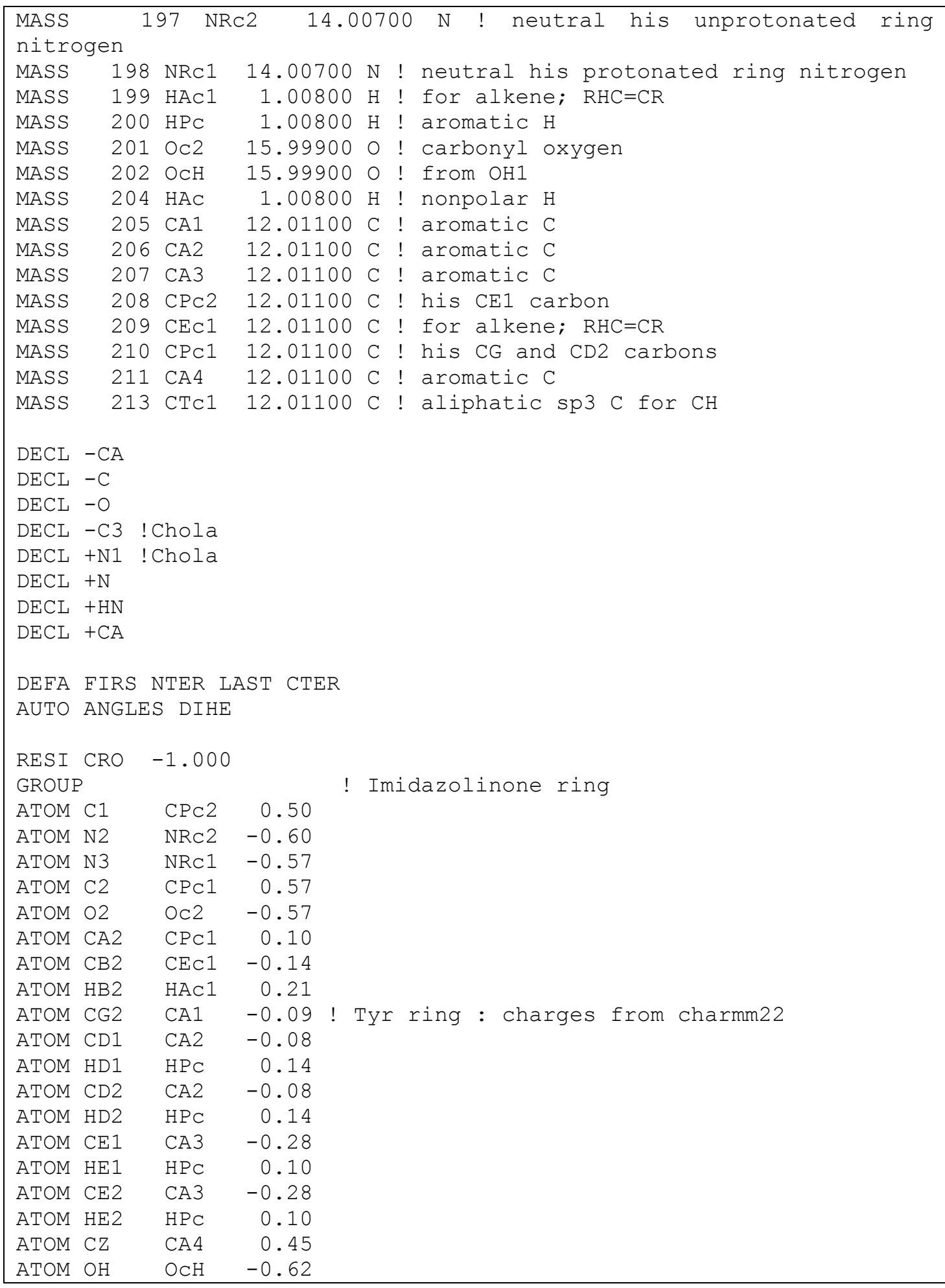




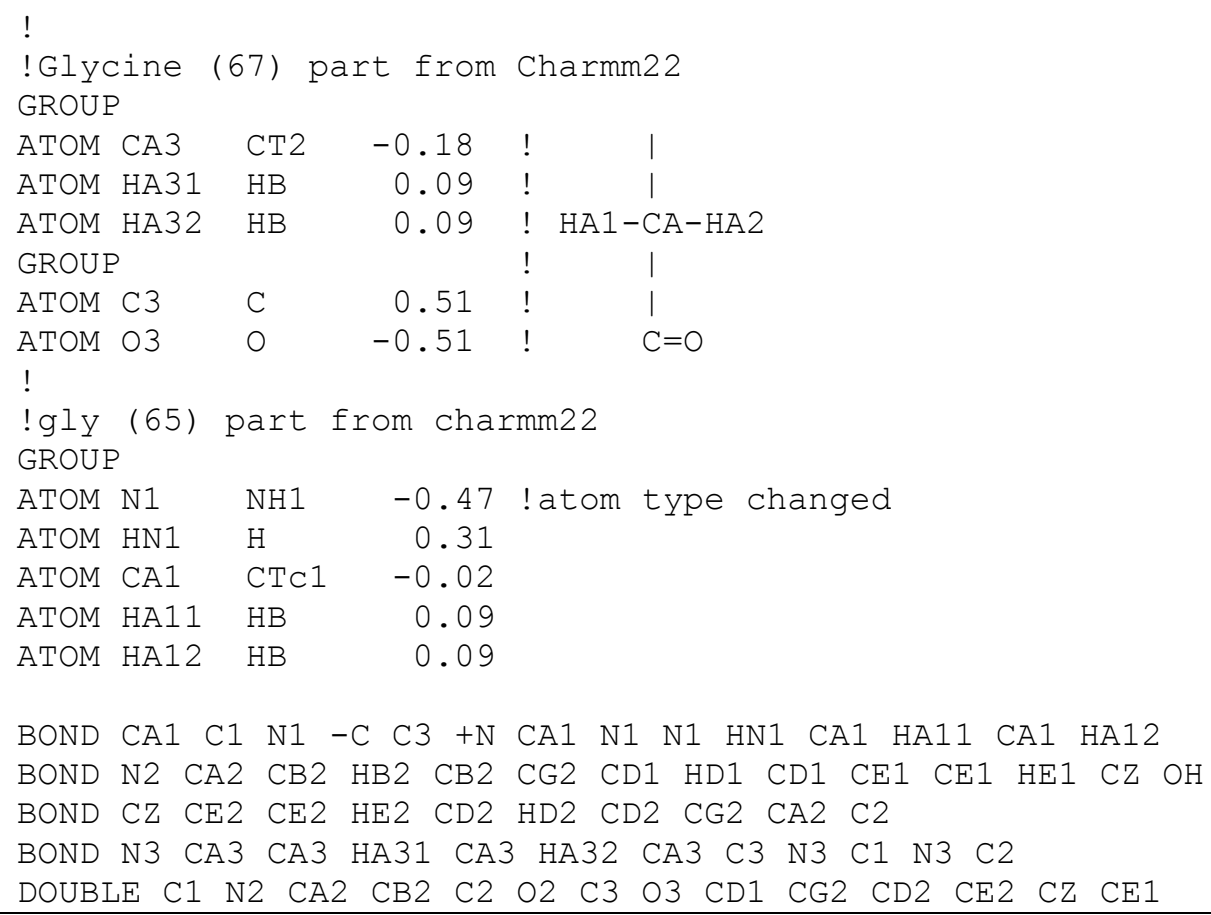

Table 5.4 Parameter file for the chromophore of citrine (anionic)

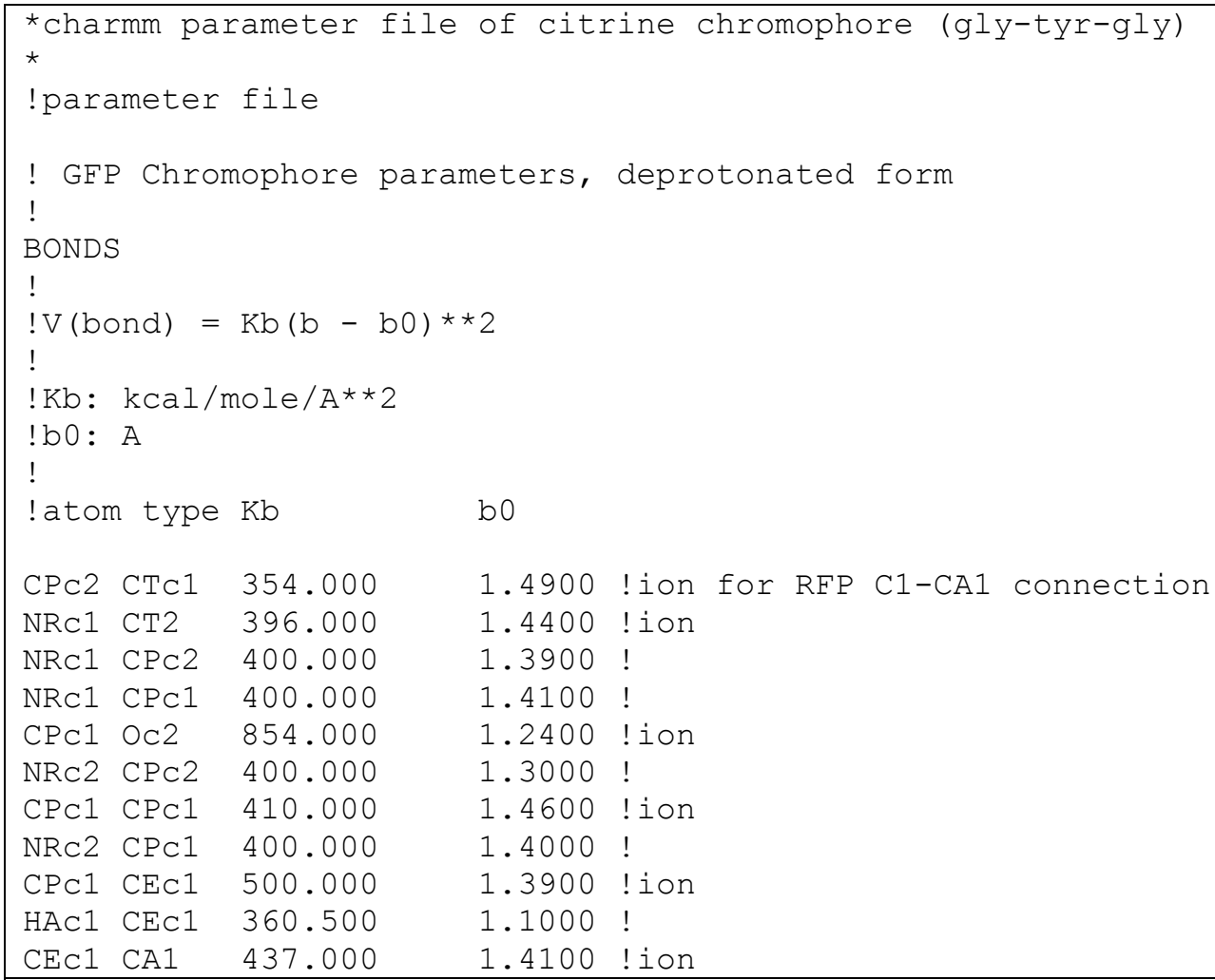




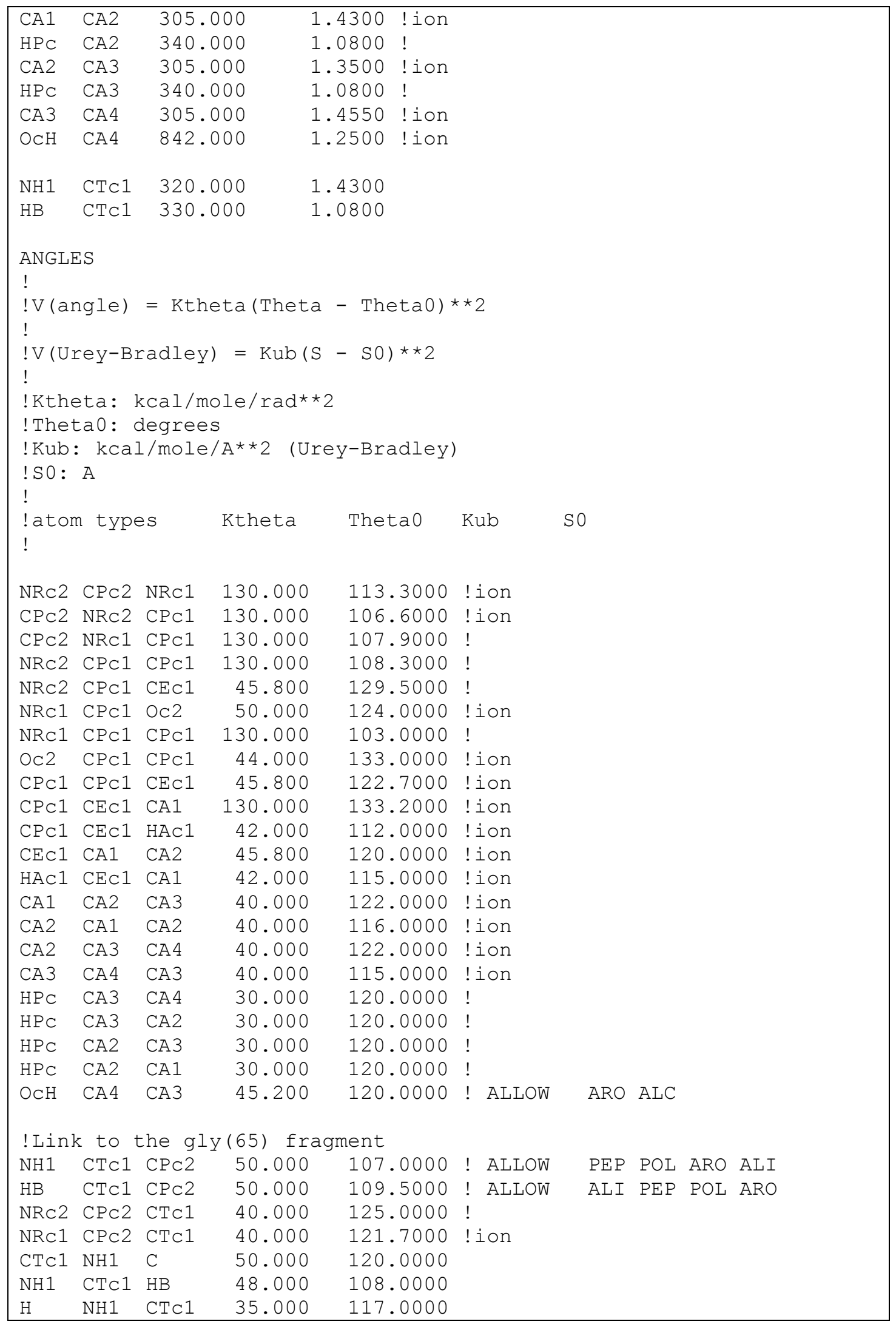




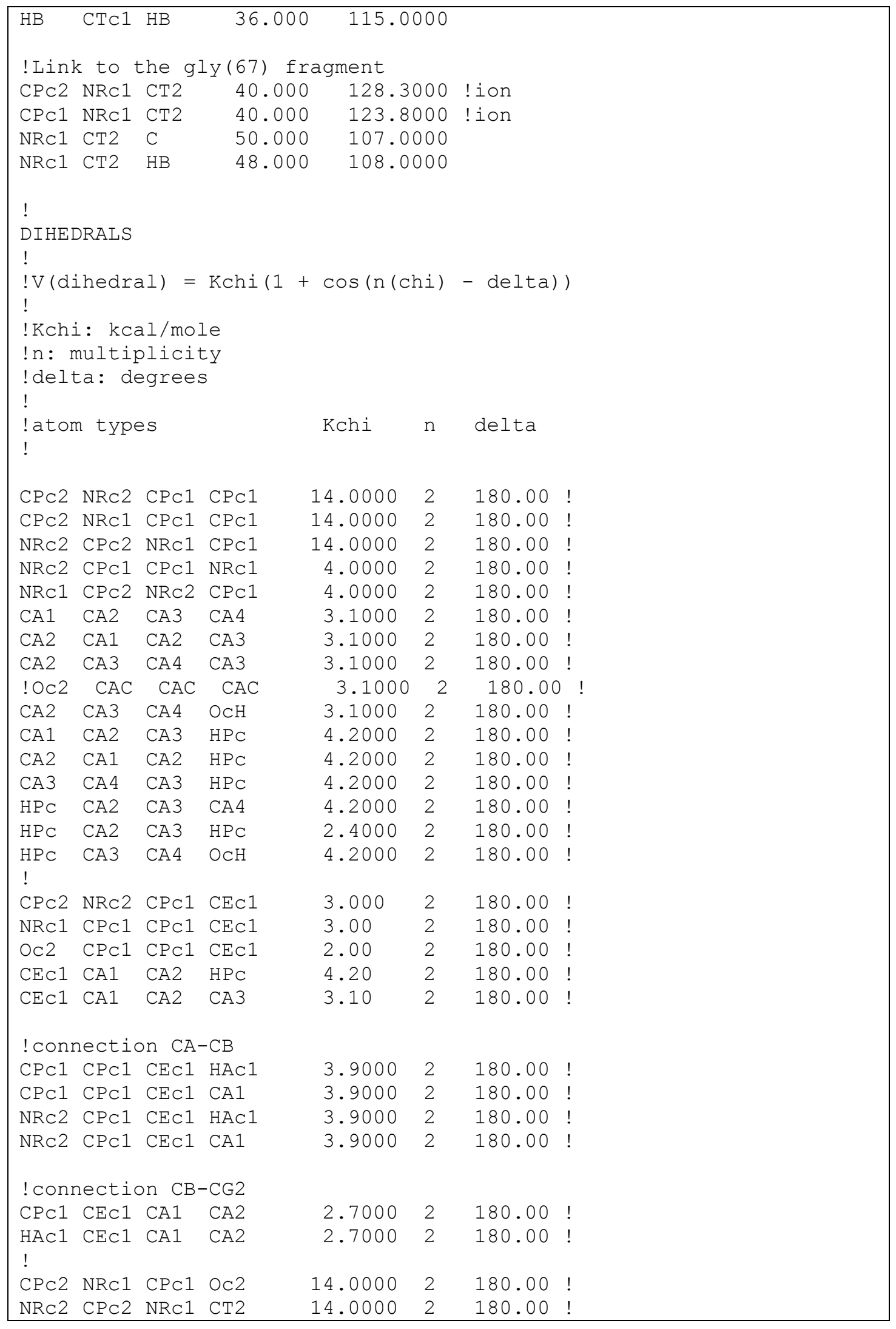




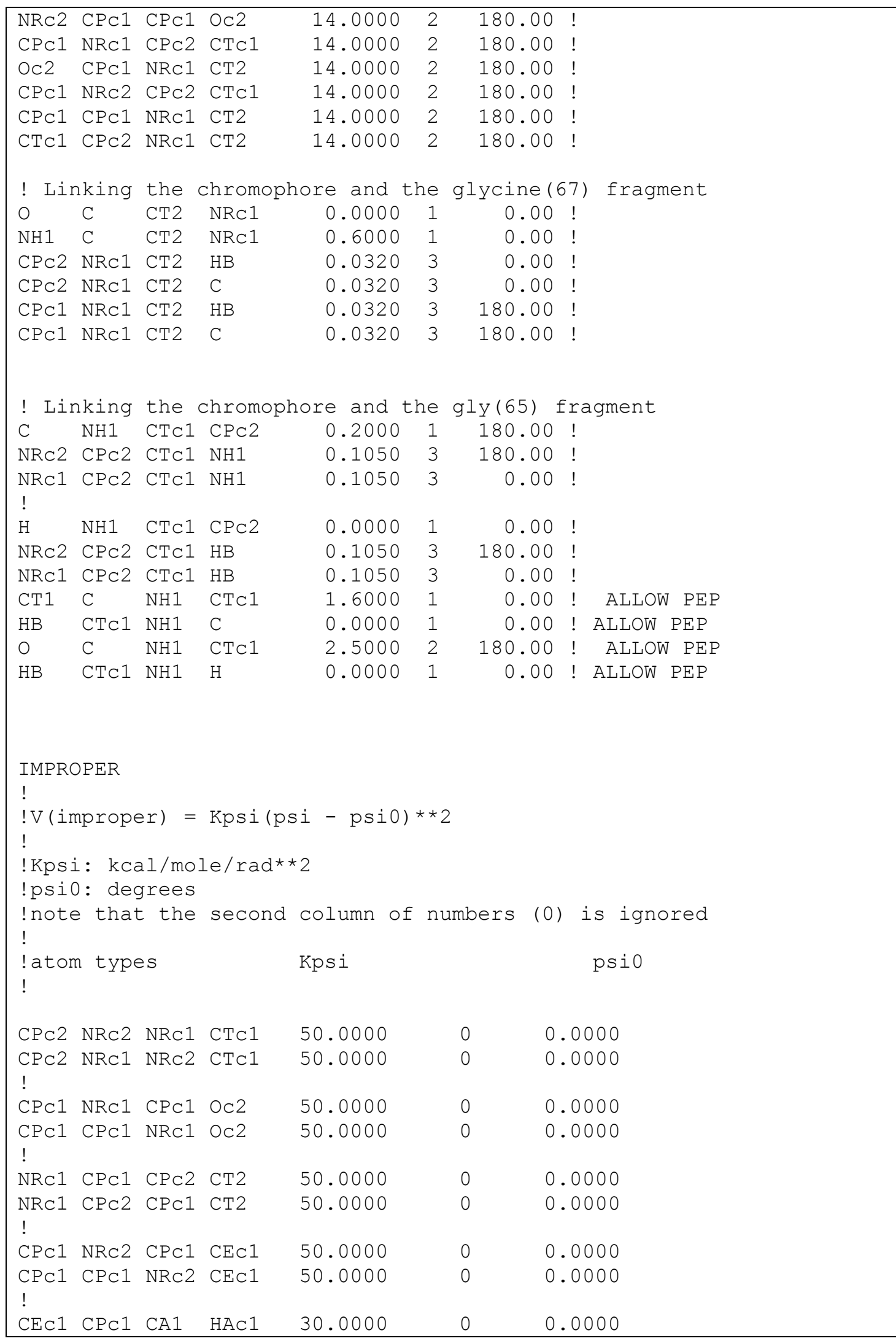




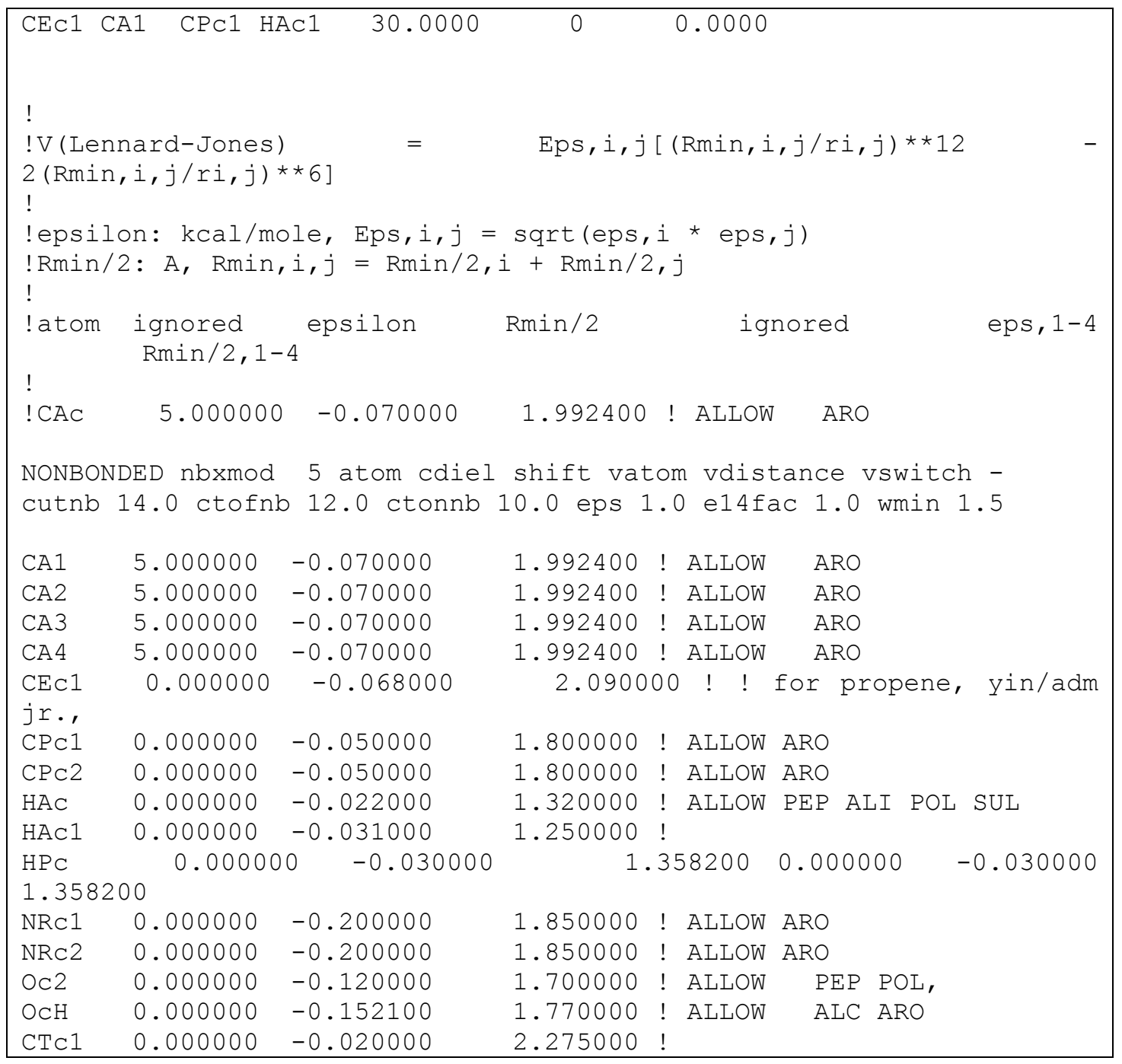

\section{Appendix D: Residue topology and parameter files for the chromophore of citrine}

Table 5.5 Residue topology file for the chromophore of citrine (neutral)

\begin{tabular}{|llrl|}
\hline RESI CRO & 0.000 & ! Imidazolinone ring \\
GROUP & & \\
ATOM C1 & CPC2 & 0.76 & \\
ATOM N2 & NRC2 & -0.55 & \\
ATOM N3 & NRC1 & -0.64 \\
ATOM C2 & CPC1 & 0.80 \\
ATOM O2 & OC2 & -0.61 \\
ATOM CA2 & CPC1 & 0.24 \\
GROUP & & \\
ATOM CB2 & CEC1 & -0.10 \\
ATOM HB2 & HAC1 & 0.10 \\
GROUP & &
\end{tabular}




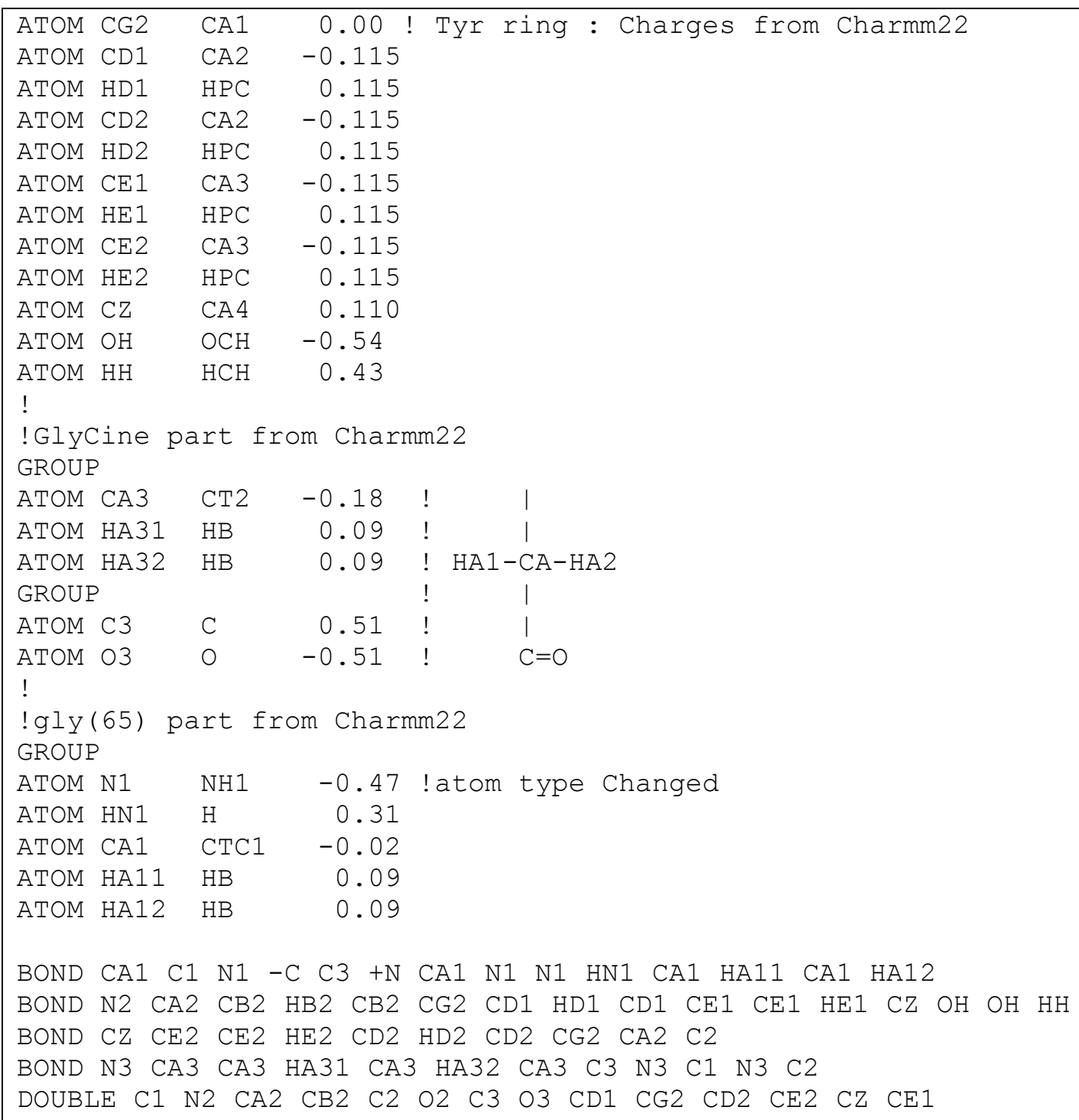

Table 5.6 Parameter file for the chromophore of citrine (neutral)

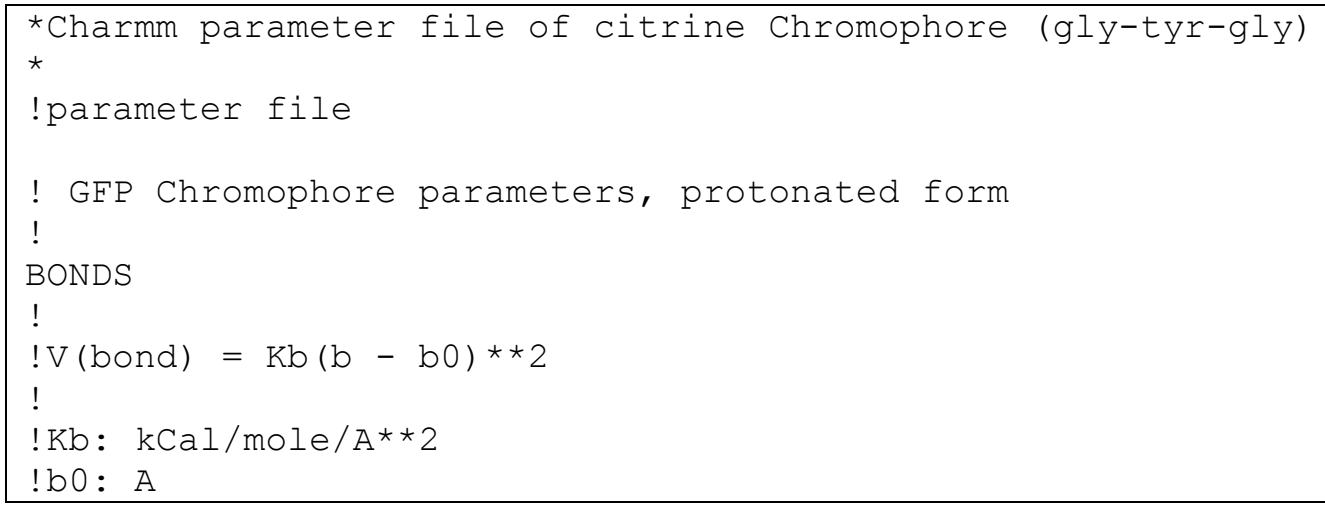




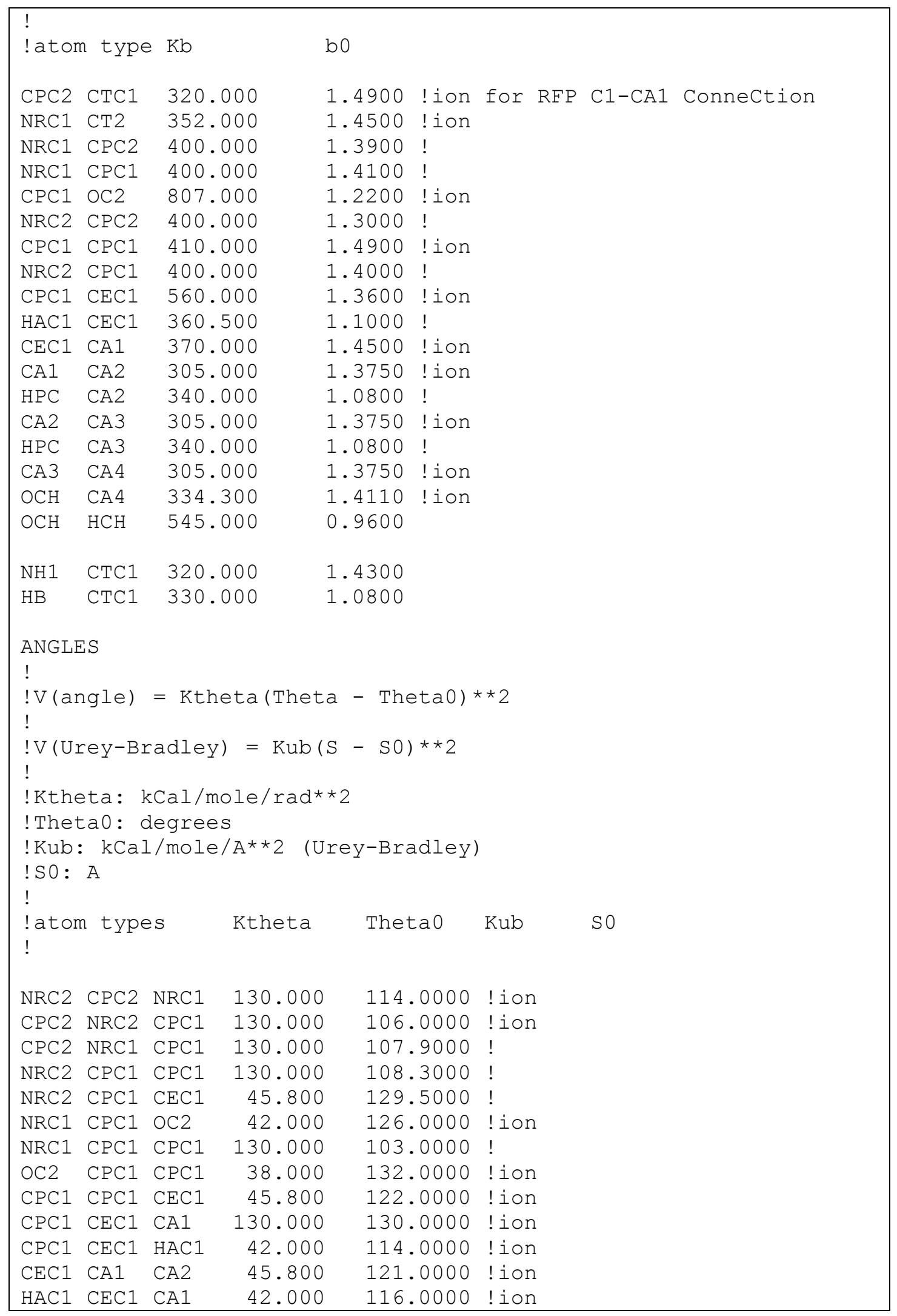




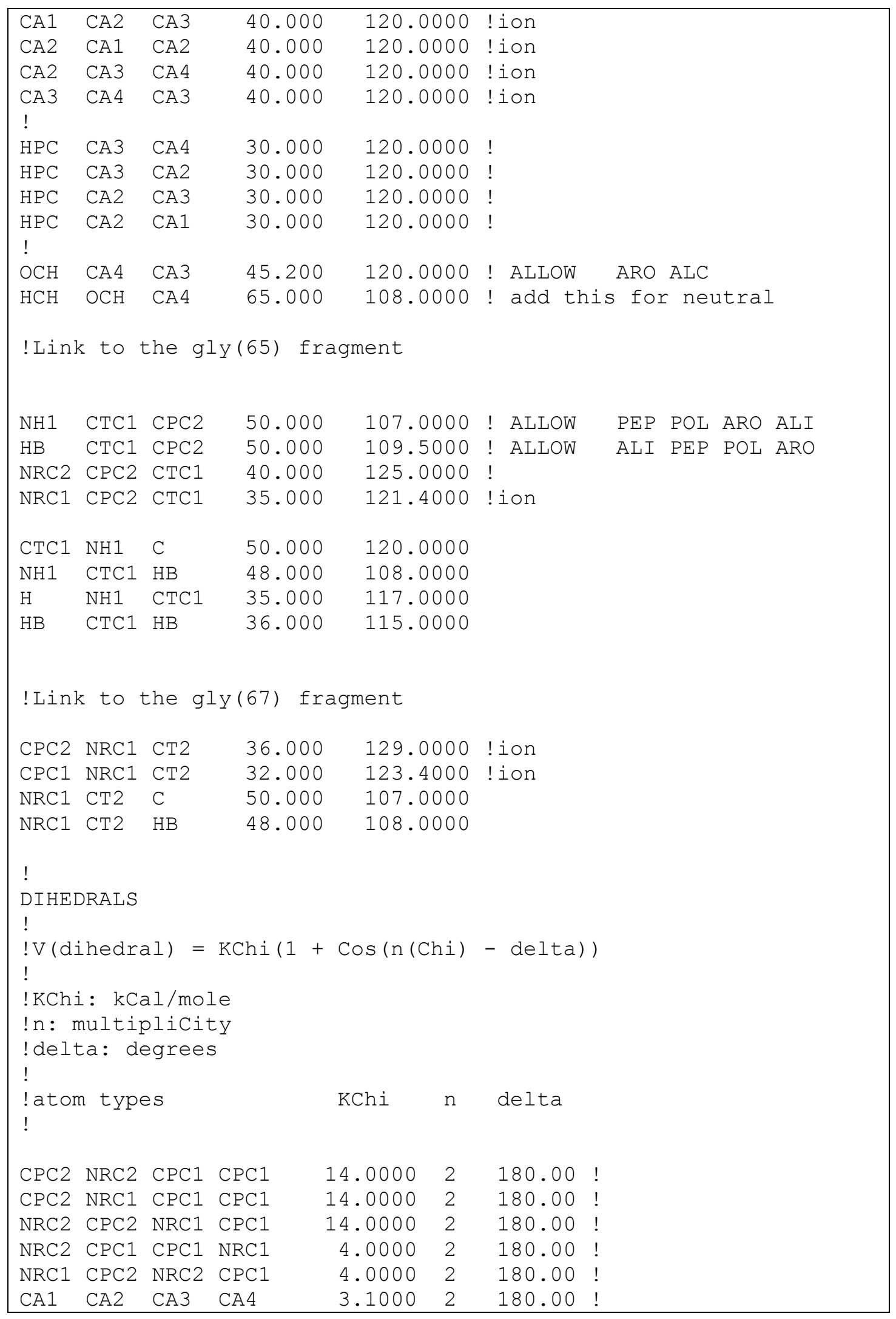




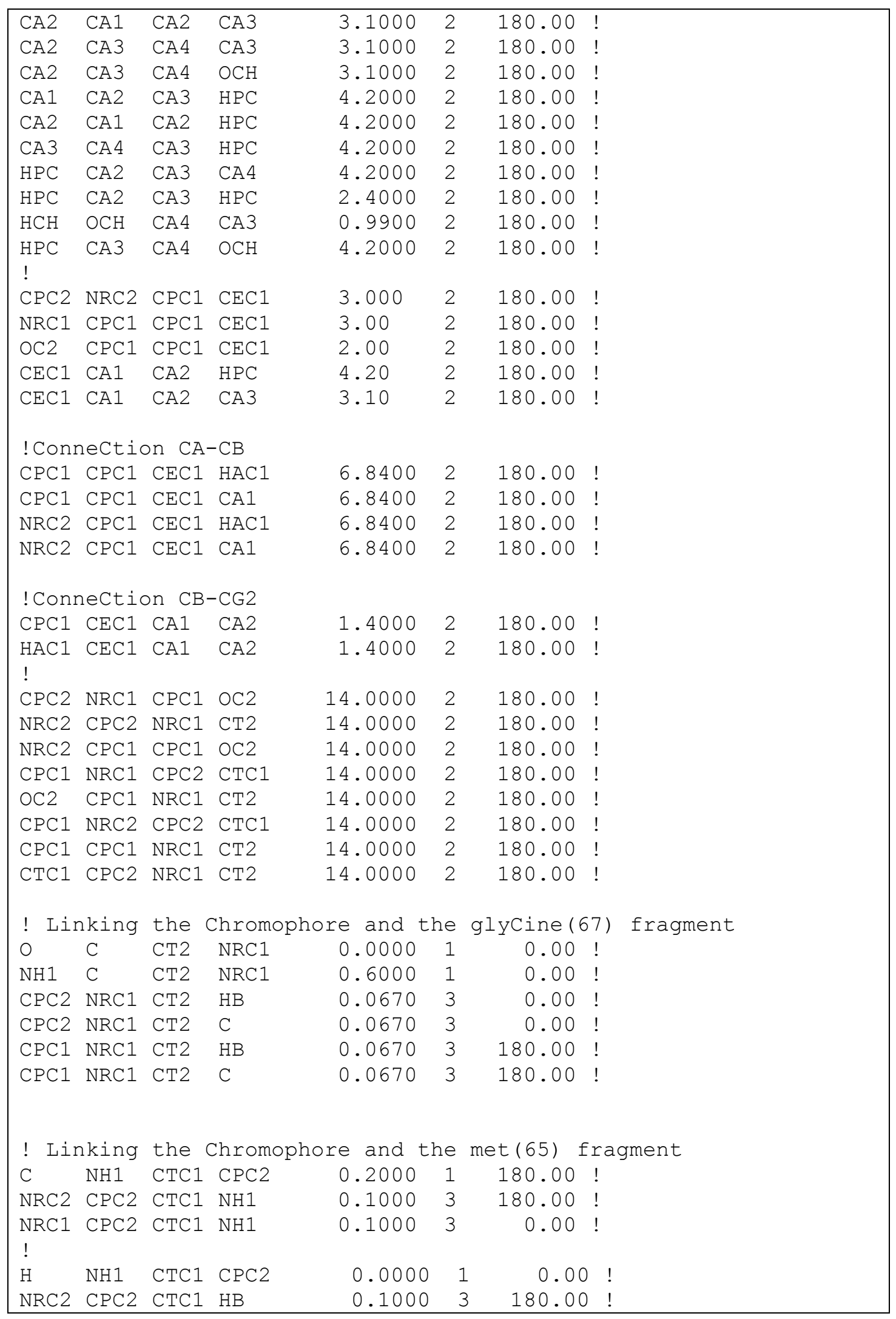




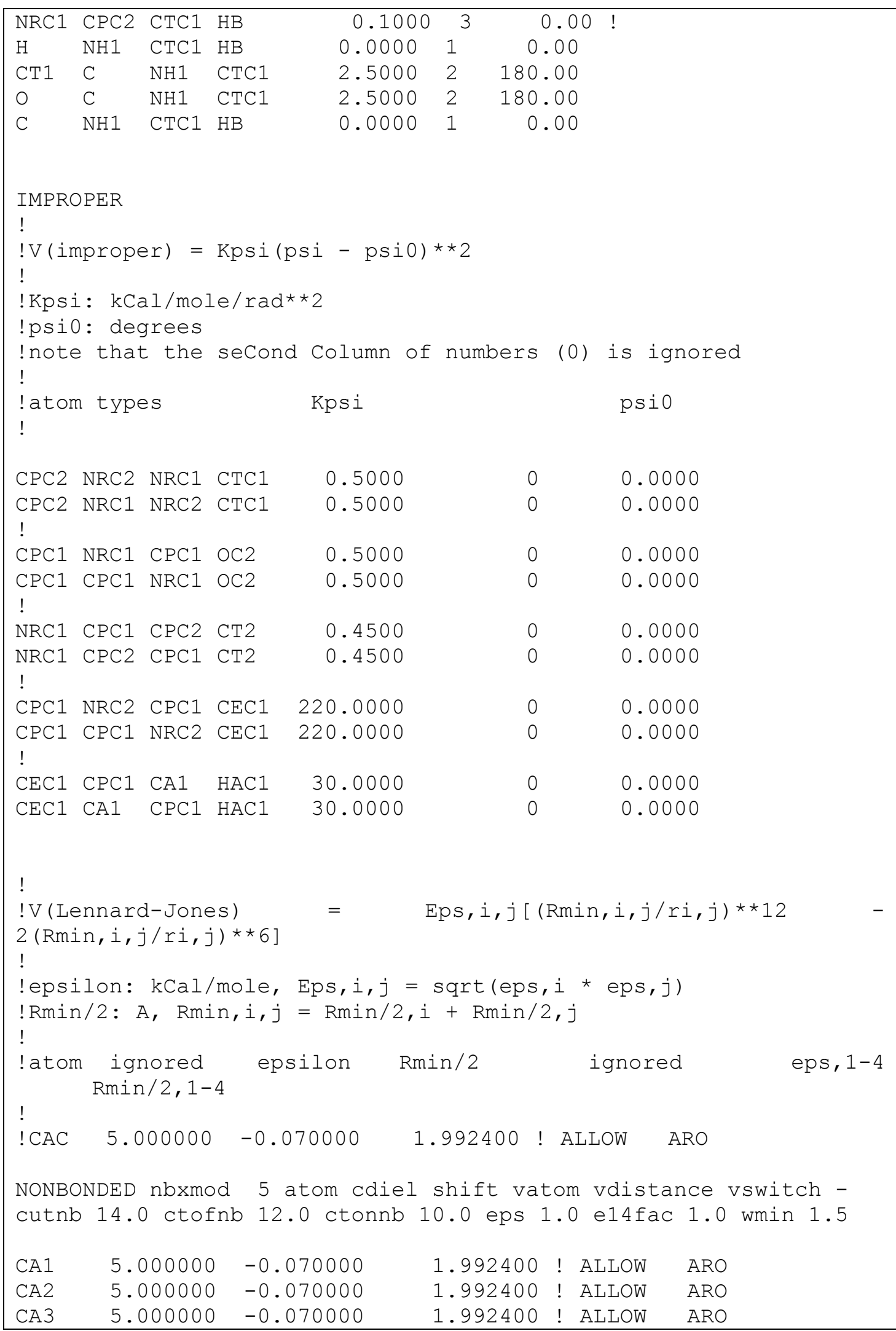




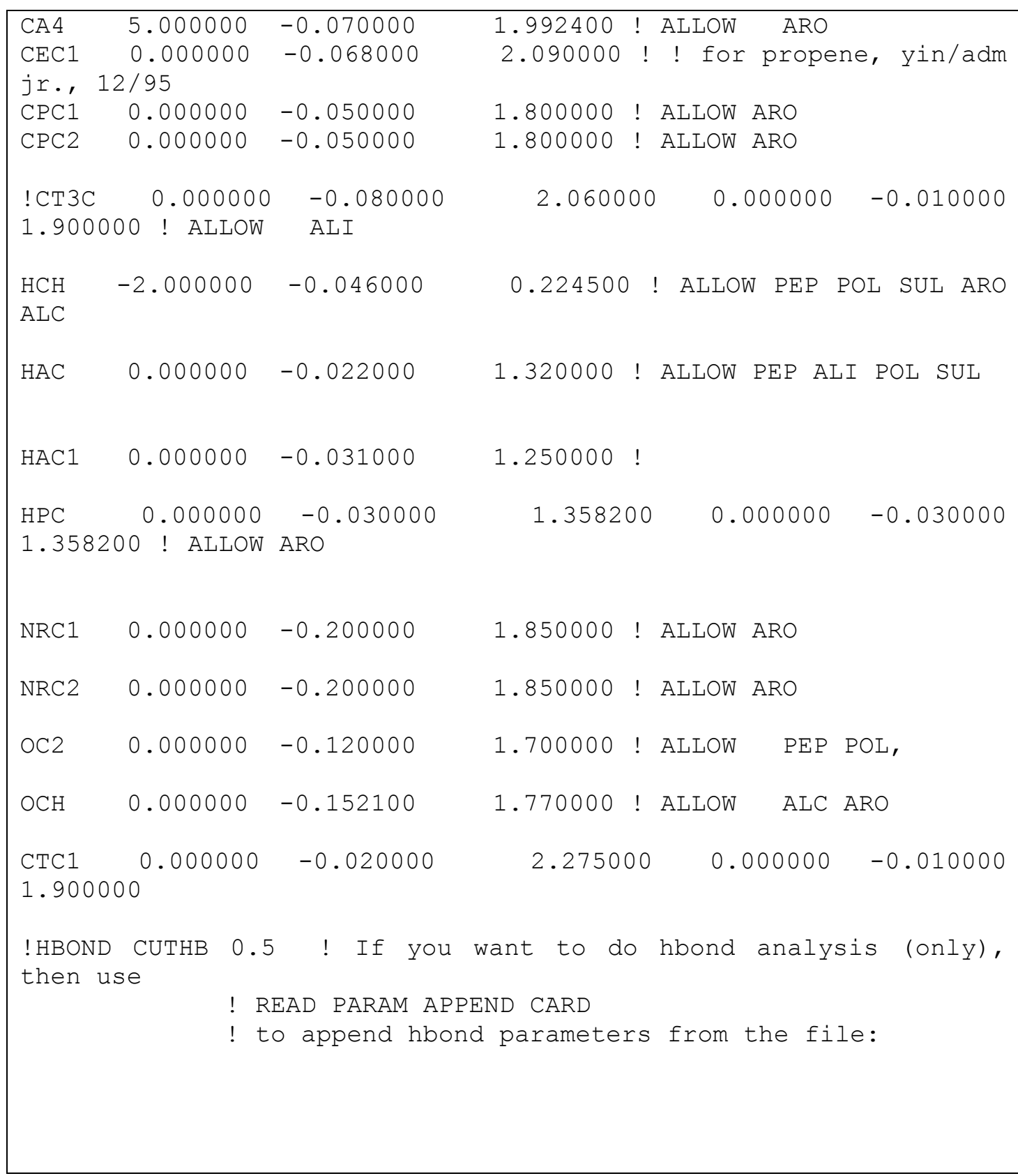

Appendix E: Residue topology and parameter files for the chromophore of mCherry

Table 5.7 Residue topology file for the chromophore of mCherry (neutral)

\begin{tabular}{|lrrr|}
\hline RESI CH6 & 0.000 & & ! Imidazolinone ring \\
GROUP & & & \\
ATOM C1 & CPC2 & 0.76 & \\
ATOM N2 & NRC2 & -0.55 & \\
ATOM N3 & NRC1 & -0.64 & \\
\hline
\end{tabular}




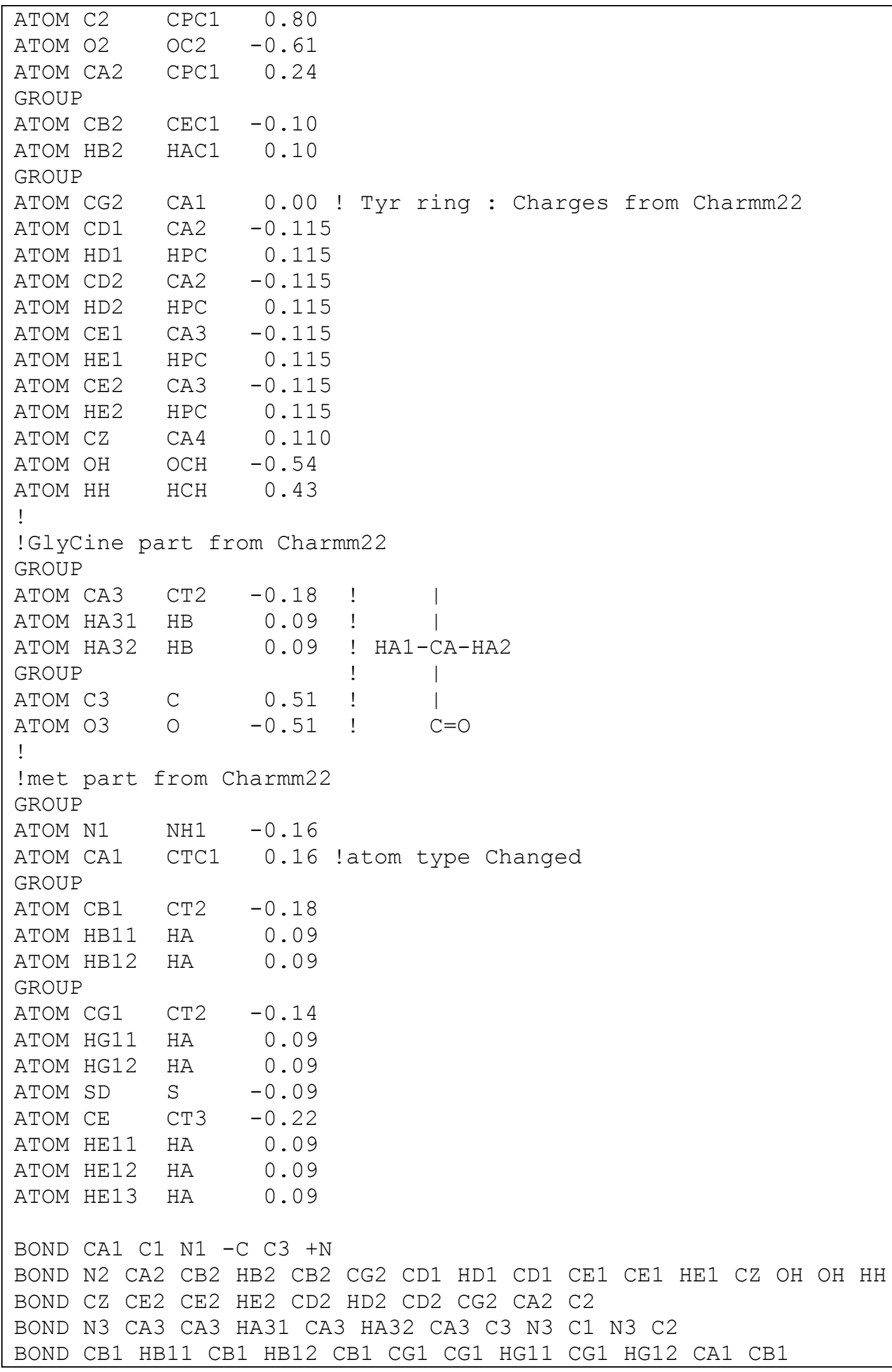


BOND CG1 SD SD CE CE HE11 CE $\mathrm{HE12}$ CE $\mathrm{HE13}$

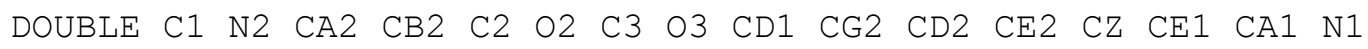

Table 5.8 Parameter file for the chromophore of mCherry (neutral)

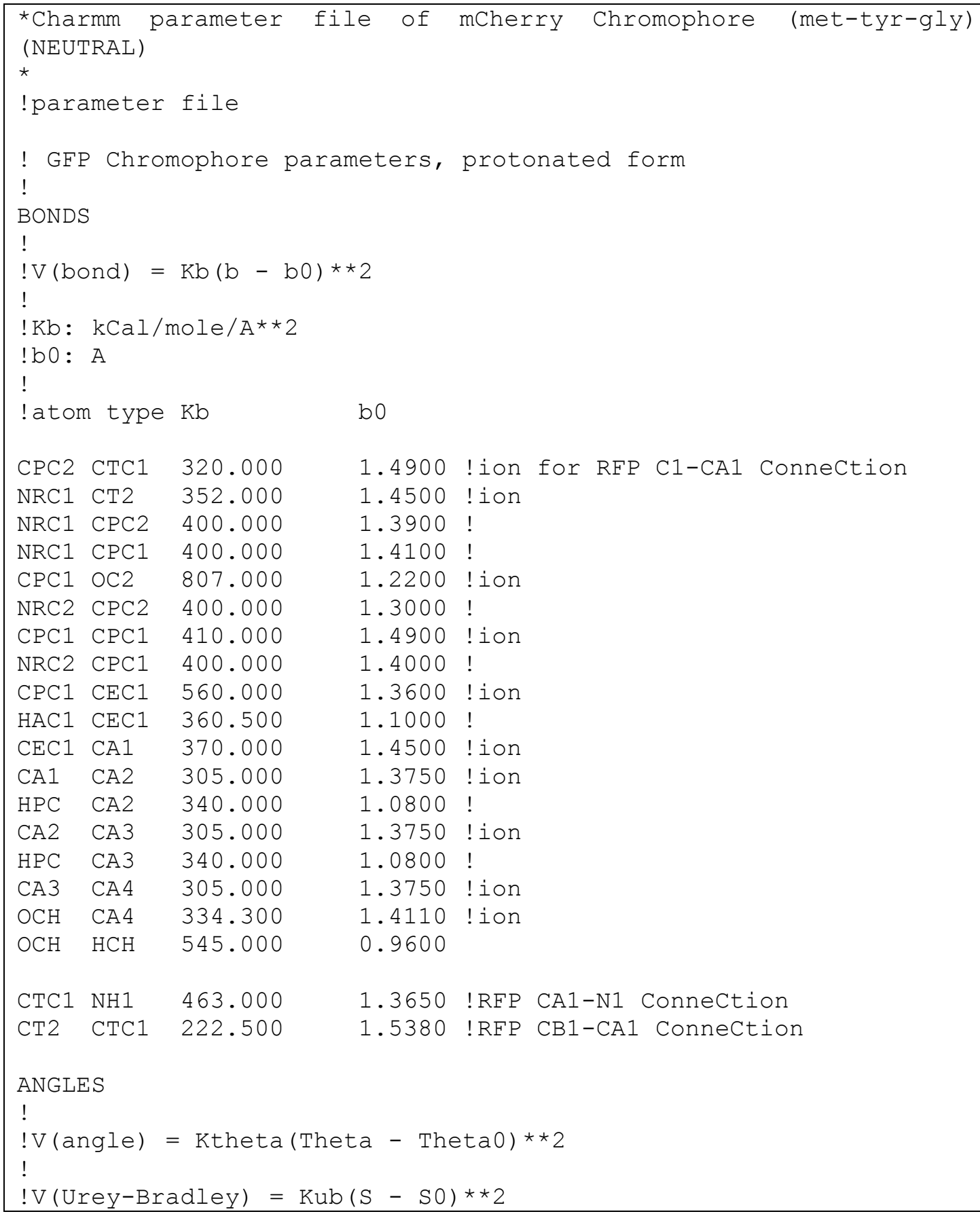




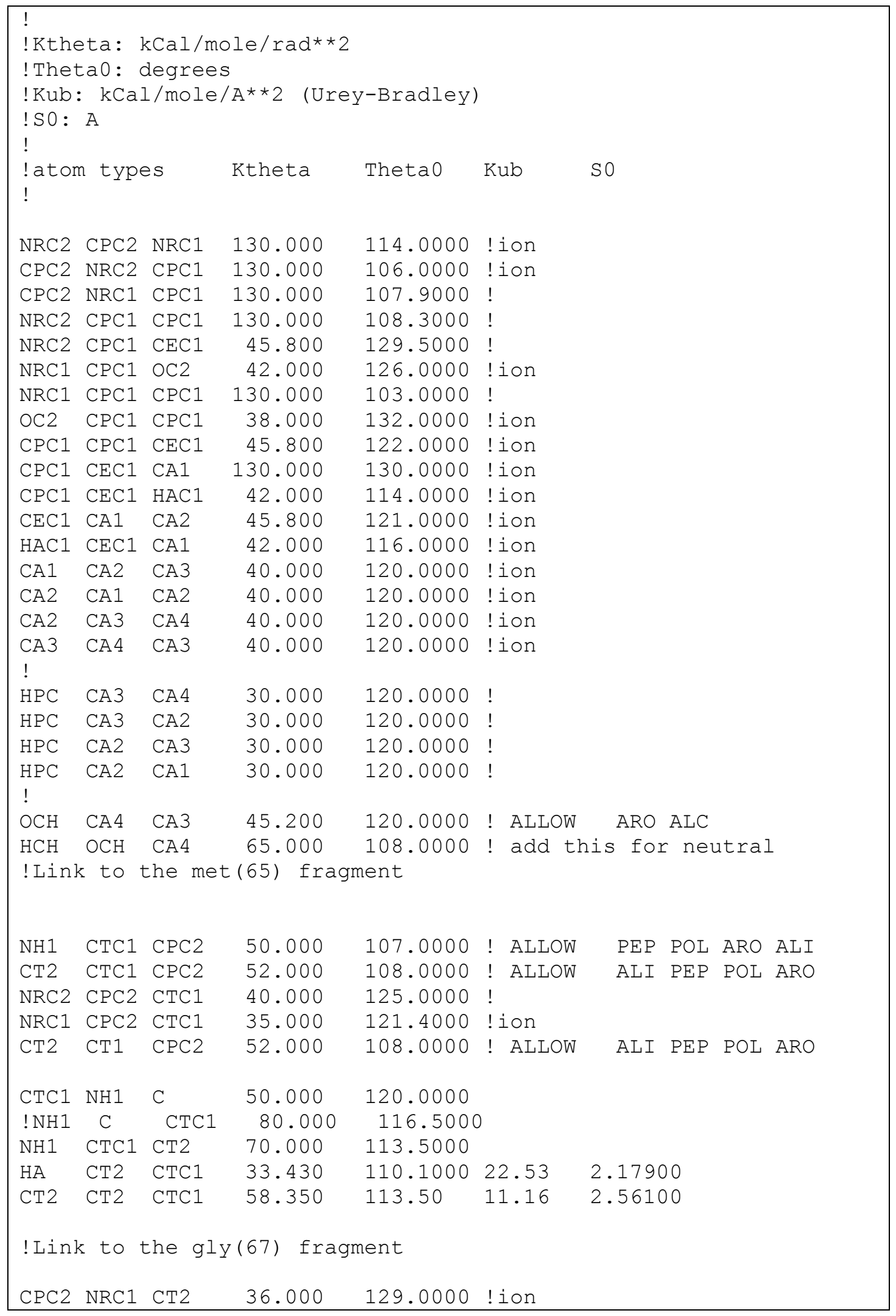




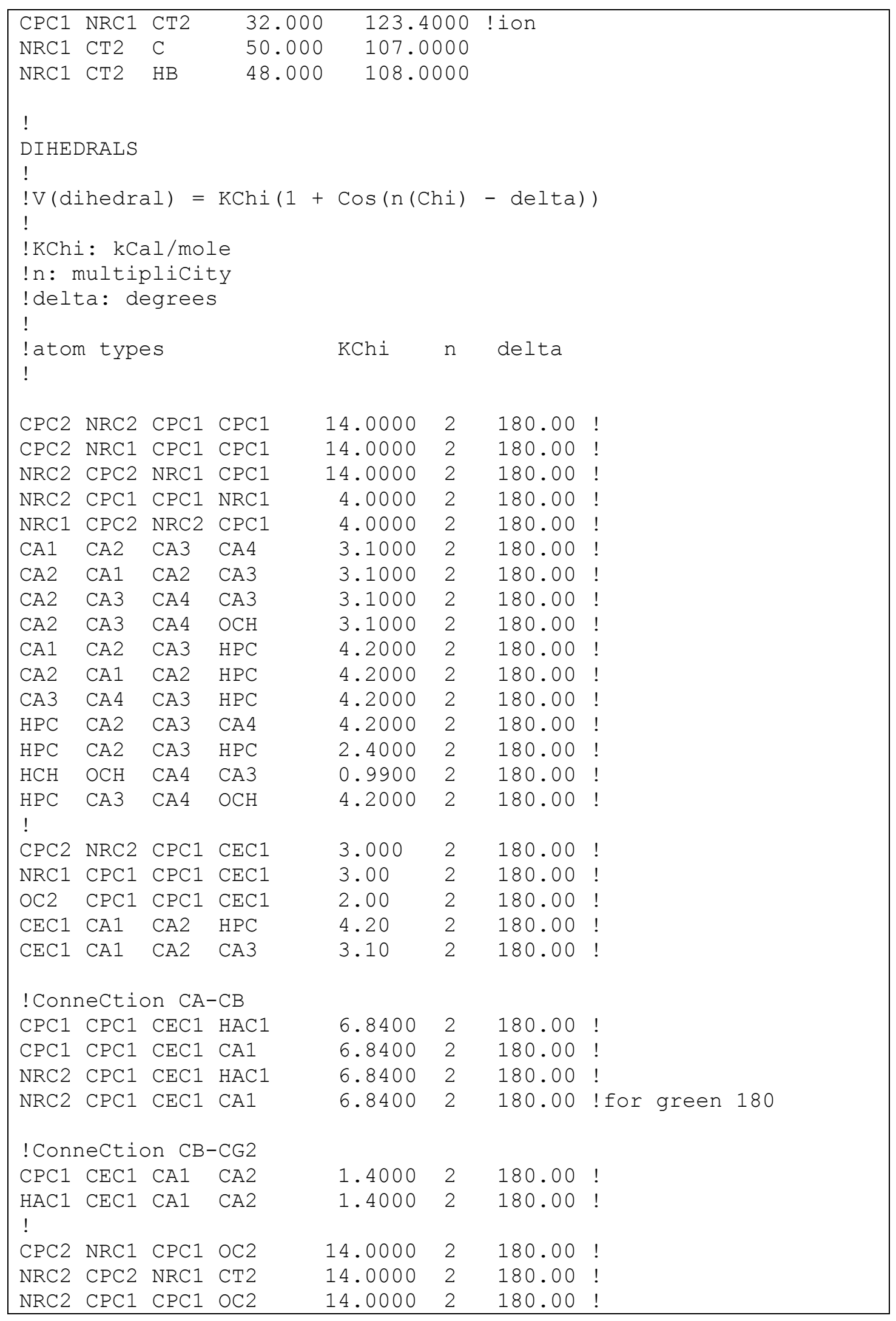




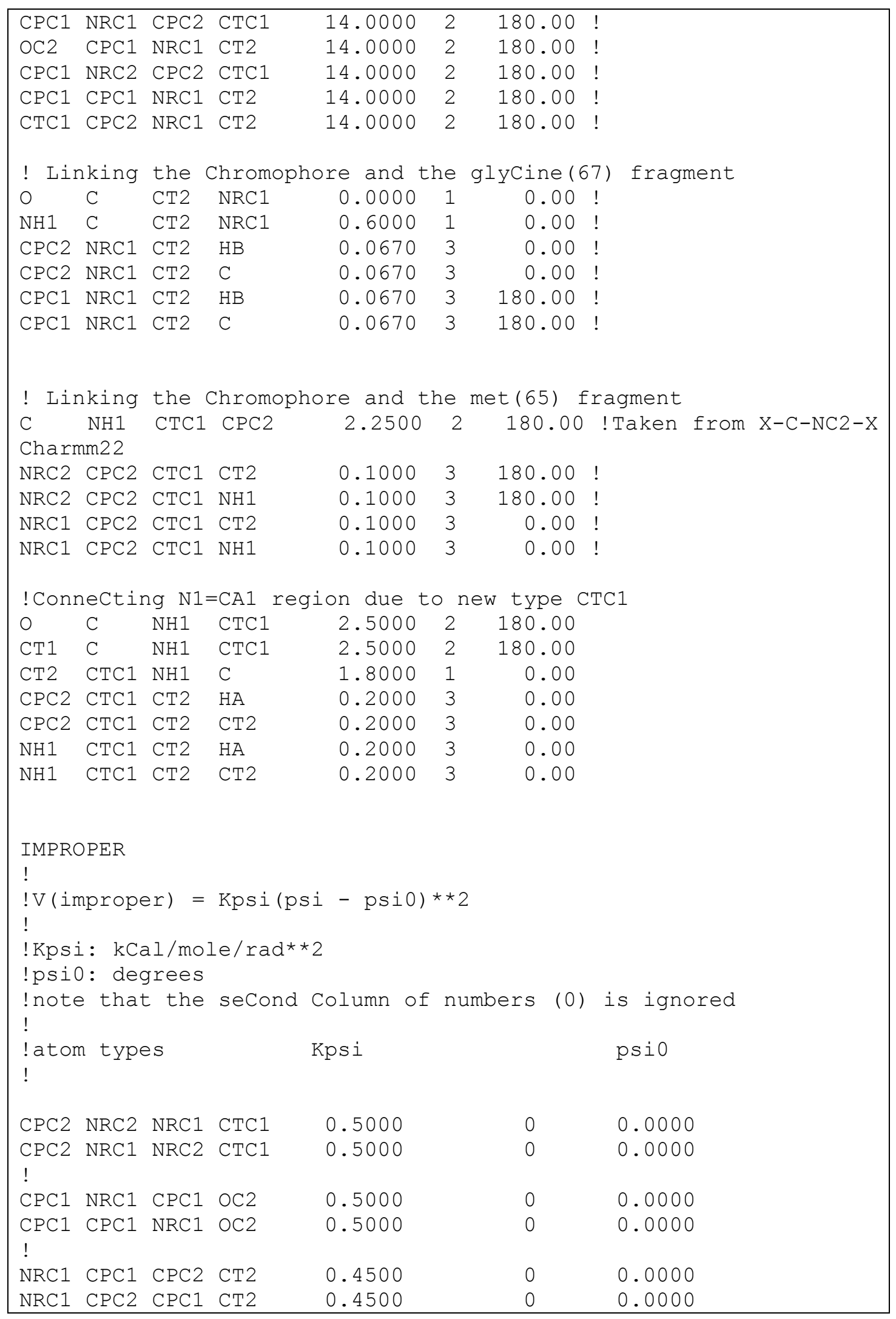




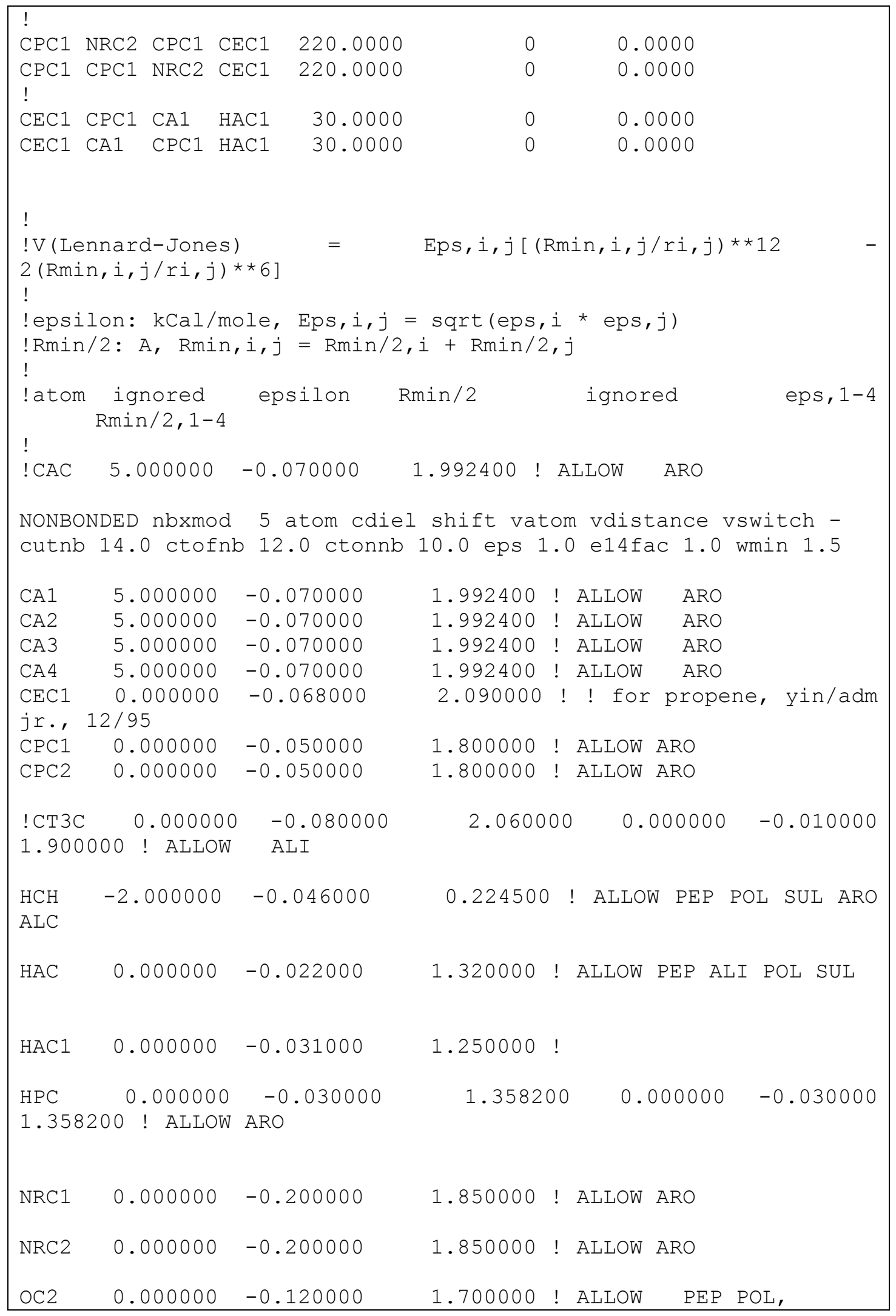




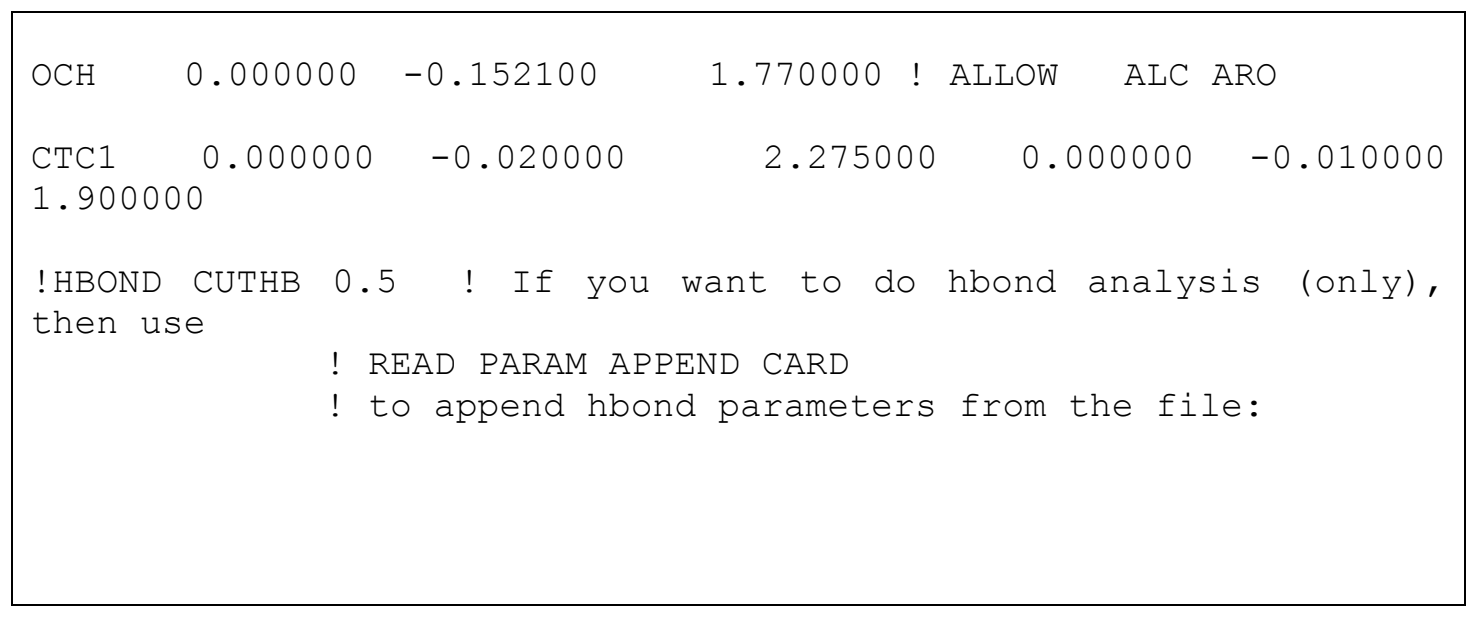


VITA

\section{CHOLA K. REGMI}

$2008-2014$

$2000-2004$

$1997-2000$

Publications:

Awards:
Graduate Teaching Assistant (Ph. D. Student) Department of Physics, Florida International University Miami, FL 33199, USA.

M. Sc. in physics, Tribhuvan University, Kathmandu, Nepal

B. Sc. in physics, Tribhuvan University, Kathmandu, Nepal

Patrick Konold, Chola K. Regmi, Prem P. Chapagain, Bernard S. Gerstman, and Ralph Jimenez, "Hydrogen Bond Flexibility Correlates with Stokes Shift in mPlum Variants", Journal of Physical Chemistry B, 2014, 118 (11), pp 2940-48

Chola K. Regmi, Yuba R. Bhandari, Bernard S. Gerstman, and Prem P. Chapagain, "Exploring the Diffusion of Molecular Oxygen in the Red Fluorescent Protein mCherry Using Explicit Oxygen Molecular Dynamics Simulations", Journal of Physical Chemistry B, 2013, 117 (8), pp 2247-53

Prem P. Chapagain, Chola K. Regmi, and Willam Castillo, "Fluorescent protein barrel fluctuations and oxygen diffusion pathways in mCherry", Journal of Chemical Physics 135 (23), 235101, 2011

New Investigator Travel Award 2014, APS Division of Chemical Physics, APS March Meeting 2014

Travel Award, 'Workshop on Multiscale Theory and Simulation', University of Chicago, Chicago, IL, June 17-19, 2013

Second Prize Award, Annual Graduate Scholarly Forum, Florida International University, Miami, FL 33199, USA, March 26, 2013

First Prize Award, Graduate Student Research Competition, Department of Physics, Florida International University, Miami, FL 33199, USA, April 19, 2012 
Presentations:

2014

2014

2013

2013

2012

2012

2011
C.Regmi, P. Chapagain, and B. Gerstman, "Temperature dependent solvation dynamics of the chromophore environment in the far-red fluorescent protein mPlum" APS March Meeting, March 03-07, 2014, Denver, CO, USA

C.Regmi, P. Chapagain, and B. Gerstman, "Temperature dependence of the protein-chromophore hydrogen bond dynamics in the far-red fluorescent protein mPlum" Biophysical Society $58^{\text {th }}$ Annual Meeting, February 15-19, 2014, San Francisco, CA, USA (Poster)

C.Regmi, "Diffusion of molecular oxygen in the red fluorescent protein mCherry" Annual Graduate Scholarly Forum, Florida International University, Miami, FL 33199, USA, March 26, 2013

C.Regmi, Y. Bhandari, P. Chapagain, and B. Gerstman, "Diffusion of molecular oxygen in the red fluorescent protein mCherry" APS March Meeting, March 18-22, 2013, Baltimore, MD, USA

C. Regmi, "Barrel fluctuations and oxygen diffusion pathways in the monomeric fluorescent proteins", Graduate Student Research Competition, Department of Physics, Florida International University, Miami, FL 33199, USA, April 19, 2012

C. Regmi, P. Chapagain, Y. Bhandari, I. Chemmama, and B. Gerstman, "Barrel fluctuations and oxygen diffusion pathways in the monomeric fluorescent proteins", APS March Meeting, Feb 27March 2, 2012, Boston, MA, USA

C. Regmi, P. Chapagain, and B. Gerstman, "Protein barrel fluctuations and the barrel permeability: A comparison between Green and Red Fluorescent Proteins", APS March Meeting, March 21-25, 2011, Dallas, TX, USA 